\title{
THE COS-HALOS SURVEY: RATIONALE, DESIGN, AND A CENSUS OF CIRCUMGALACTIC NEUTRAL HYDROGEN*
}

\author{
Jason Tumlinson $^{1}$, Christopher Thom $^{1}$, Jessica K. Werk ${ }^{2}$, J. Xavier Prochaska ${ }^{2}$, Todd M. Tripp ${ }^{3}$, Neal Katz ${ }^{3}$, \\ Romeel Davé ${ }^{4,5}$, Benjamin D. Oppenheimer ${ }^{6,7}$, Joseph D. Meiring ${ }^{3}$, Amanda Brady Ford ${ }^{8}$, John M. O’Meara ${ }^{9}$, \\ Molly S. Peeples ${ }^{10}$, Kenneth R. Sembach ${ }^{1}$, and David H. Weinberg ${ }^{11}$ \\ ${ }^{1}$ Space Telescope Science Institute, Baltimore, MD, USA \\ ${ }^{2}$ UCO/Lick Observatory, University of California, Santa Cruz, CA, USA \\ ${ }^{3}$ Department of Astronomy, University of Massachusetts, Amherst, MA, USA \\ ${ }^{4}$ University of the Western Cape, South African Astronomical Observatories, and African Institute for Mathematical Sciences, Cape Town, South Africa \\ ${ }_{5}^{5}$ Astronomy Department, University of Arizona, Tucson, AZ 85721, USA \\ ${ }^{6}$ Leiden Observatory, Leiden University, 2300 RA Leiden, The Netherlands \\ ${ }^{7}$ CASA, Department of Astrophysical and Planetary Sciences, University of Colorado, Boulder, CO 80309, USA \\ ${ }^{8}$ Steward Observatory, University of Arizona, Tucson, AZ, USA \\ ${ }^{9}$ Department of Chemistry and Physics, Saint Michael's College, Colchester, VT, USA \\ ${ }^{10}$ Center for Galaxy Evolution, University of California Los Angeles, Los Angeles, CA, USA \\ ${ }^{11}$ Department of Astronomy, The Ohio State University, Columbus, OH, USA \\ Received 2013 May 30; accepted 2013 September 9; published 2013 October 17
}

\begin{abstract}
We present the design and methods of the COS-Halos survey, a systematic investigation of the gaseous halos of $44 z=0.15-0.35$ galaxies using background QSOs observed with the Cosmic Origins Spectrograph aboard the Hubble Space Telescope. This survey has yielded 39 spectra of $z_{\mathrm{em}} \simeq 0.5$ QSOs with $\mathrm{S} / \mathrm{N} \sim 10-15$ per resolution element. The QSO sightlines pass within 150 physical kpc of the galaxies, which span early and late types over stellar mass $\log M_{*} / M_{\odot}=9.5-11.5$. We find that the circumgalactic medium exhibits strong $\mathrm{H}$, averaging $\simeq 1 \AA$ in $\mathrm{Ly} \alpha$ equivalent width out to $150 \mathrm{kpc}$, with $100 \%$ covering fraction for star-forming galaxies and $75 \%$ covering for passive galaxies. We find good agreement in column densities between this survey and previous studies over similar range of impact parameter. There is weak evidence for a difference between early- and late-type galaxies in the strength and distribution of H I. Kinematics indicate that the detected material is bound to the host galaxy, such that $\gtrsim 90 \%$ of the detected column density is confined within $\pm 200 \mathrm{~km} \mathrm{~s}^{-1}$ of the galaxies. This material generally exists well below the halo virial temperatures at $T \lesssim 10^{5} \mathrm{~K}$. We evaluate a number of possible origin scenarios for the detected material, and in the end favor a simple model in which the bulk of the detected H I arises in a bound, cool, low-density photoionized diffuse medium that is generic to all $L^{*}$ galaxies and may harbor a total gaseous mass comparable to galactic stellar masses.
\end{abstract}

Key words: galaxies: formation - galaxies: halos - intergalactic medium - quasars: absorption lines

Online-only material: color figures, figure set

\section{INTRODUCTION AND MOTIVATIONS}

The means by which galaxies acquire their gas, process it into stars, and expel it as energetic feedback have assumed central importance in the modern picture of galaxy formation. Solutions to important puzzles such as the galactic "missing baryons" problem, the mass-metallicity relation, and the color-magnitude bimodality must involve the flows of gas that cycle through the intergalactic medium (IGM), circumgalactic medium (CGM), and interstellar medium (ISM) during galaxy evolution. Those parts of galaxies that are readily visible in emission-stars and the ISM gas they illuminate-constitute the outcome of the flows from the IGM and CGM, not those flows themselves. At least in the IGM and CGM, these flows are difficult to observe directly because the gas is diffuse and spread over large regions of space. Fortunately, absorption-line techniques can access physical tracers at the relevant densities with extremely high sensitivity.

This paper describes the properties of a new survey ("COS-Halos") of the CGM gas surrounding a sample of

\footnotetext{
* Based on observations made with the NASA/ESA Hubble Space Telescope, obtained at the Space Telescope Science Institute, which is operated by the Association of Universities for Research in Astronomy, Inc., under NASA contract NAS 5-26555. These observations are associated with program GO11598.
}

$L \sim L^{*}$ galaxies in the low-redshift Universe using the Cosmic Origins Spectrograph (COS) aboard the Hubble Space Telescope $(H S T)$. The primary motivation for the COS-Halos survey is to examine the content of the CGM and to better understand its role in galaxy formation. The design of the survey leverages the large advance in UV spectroscopic sensitivity offered by COS (Green et al. 2012) to address the CGM with a larger sample and better control over galaxy populations than was possible with earlier instruments and selection techniques. As a result of the improvement in sensitivity, it has become feasible to choose a sample of galaxies with particular properties and to observe their halo gas in a commonly studied suite of hydrogen and metal-line diagnostics. The major scientific motivations for this survey are:

Galaxy accretion-hot, cold, and multiphase. The question of how galaxies acquire their gas dates back at least to White $\&$ Rees (1978), who posited that gas entering a halo shock heats to roughly the virial temperature $\left(T \sim 10^{6} \mathrm{~K}\right.$ for a Milky Way (MW) sized galaxy), before cooling and collapsing to form the central galaxy. This basic picture has been modified in many ways and has reached its fullest development in detailed hydrodynamical simulations that track gas infall, cooling, star formation, and feedback self-consistently within a cosmological context. When these details are included 
as faithfully as possible in simulations, a theoretical picture emerges that is more complex than the traditional picture in which all gas shock-heats to the halo virial temperature before cooling and falling, more or less spherically, into the galaxy.

The "bimodal accretion" scenario posits two primary modes by which gas can accrete. Galaxies with $M_{*} \gtrsim 5 \times 10^{10} M_{\odot}$ reside in halos massive enough at $z \sim 0$ to accrete through the "hot mode," with gas shock-heated near the halo virial radius $\left(R_{\text {vir }}\right)$ to the halo virial temperature $\left(T_{\text {vir }} \sim 10^{6} \mathrm{~K}\right)$. This accretion mode closely resembles the earlier shocked-accretion spherical inflow scenario. For galaxies at $M_{*} \lesssim 5 \times 10^{10} M_{\odot}$, rapid radiative cooling removes the pressure needed to maintain the shock and gas can penetrate far inside $R_{\text {vir }}$ without heating above $\sim 10^{4}-10^{5} \mathrm{~K}$ as it accretes along narrow filaments (Kereš et al. 2005; Dekel \& Birnboim 2006). This is the "cold mode" of accretion. The predicted transition halo mass between the hot and cold modes of accretion lies near the observed transition between high-mass, red, bulgy galaxies and low-mass, blue, disky galaxies, so it is tempting to associate the two phenomena and thus explain the bimodality of galaxy colors in terms of gas accretion. Recent simulations based on new treatments of hydrodynamics have questioned the details of cold accretion predictions (Sijacki et al. 2012; Bird et al. 2013), but in any case it is important that observations provide tests of any proposed accretion scenarios.

The "multiphase" accretion scenario (Mo \& Miralda-Escude 1996; Maller \& Bullock 2004) instead posits the existence of the hot, diffuse gaseous halo left over from virialization or a major merger, and then considers the behavior of density fluctuations that cool within this hot medium. In contrast to the canonical picture in which hot gas within $R_{\text {vir }}$ cools from the inside out (starting at small radii where the densities are highest and cooling times are shortest), in the multiphase model cooling proceeds unevenly and $T \sim 10^{4} \mathrm{~K}$ fragments form in rough pressure equilibrium with the hot diffuse halo. These cooled fragments then spiral in and form the primary means by which gas accretes to the central galaxy.

This "multiphase" picture draws support, and COS-Halos is also motivated by, the widespread detection of ionized gas and colder clouds within the hot medium surrounding the MW, as traced by the population of high-velocity clouds (HVCs; Sembach et al. 2003; Fox et al. 2006; Collins et al. 2003). The widespread HVC O vi was interpreted by Sembach et al. (2003) as collisionally ionized material at interfaces between cooler infalling clouds seen as the classical HVC complexes and a hot $\left(\sim 10^{7} \mathrm{~K}\right)$, extended ( $\left.\gtrsim 70 \mathrm{kpc}\right)$ Galactic halo. In this model, the $\mathrm{HVC} \mathrm{O}$ VI is an indirect indicator of the hot Galactic corona gas rather than a major halo component in its own right, at least within $\sim 10 \mathrm{kpc}$ of the Galactic disk (Howk \& Consiglio 2012). We were motivated to design COS-Halos in part to provide some comparisons to the MW halo gas by exploiting the very different viewing geometry possible for external galaxies. In particular, the FUSE results on Galactic halo O vi Sembach et al. (2003) was a key motivation for our choice to design COS-Halos around galaxies with sufficiently high redshift for COS to measure their O VI.

All of these considerations about galaxy accretion motivated us to design a survey that would examine CGM gas over a range of galaxy stellar masses spanning the expected transition from cold to hot accretion ( $\sim 3-5 \times 10^{10} M_{\odot}$, or $L \sim L^{*}$ galaxies), and to use ionization diagnostics that could be compared directly to MW O VI absorption and to the samples of absorbers in which
$\mathrm{O}$ VI is detected in association with diffuse $\mathrm{HI}$ and sometimes other metals (e.g., Tripp et al. 2008; Thom \& Chen 2008).

Mass, physical phases, and metal content of the CGM and IGM. The COS-Halos survey is also motivated by a desire to assess the total mass of gas in the gaseous halos of galaxies. Measuring the mass of the CGM addresses at least three key problems in galaxy evolution. First, this mass reservoir is a potential source of fuel for star formation in galaxies, which generally have short gas consumption times compared to the duration of their star formation histories. Second, the mass in the CGM may help to explain the deficiency of galactic baryon budgets with respect to their dark matter (DM) halos, if a significant budget of baryons resides in the CGM. Third, the baryonic mass in the CGM may be a significant reservoir of cosmic baryons, which are undercounted at low redshift (Persic \& Salucci 1992; Fukugita et al. 1998; Cen \& Ostriker 1999; Prochaska et al. 2011b) but which may reside in diffuse ionized gas within galaxy halos (Bregman 2007). All these problems can be addressed by a survey that can estimate the total quantities of neutral and ionized gas within $R_{\text {vir }}$ of lowredshift galaxies. These considerations drive the survey toward a design that gives good constraints on the total gas column densities and ionization corrections derived by the best available metal-line ionization diagnostics, for galaxies over a range of stellar mass (as a proxy for DM halo mass). Such a survey can also assess the CGM mass as a function of gas temperature and/or density, if appropriate diagnostics are available. It is important to understand the physical state of the gas to avoid double-counting baryons already in the census, such as might occur if they are highly overlapping with the metal-enriched, mostly photoionized absorbers in the Ly $\alpha$ or O vi phases of the IGM (Thom \& Chen 2008; Tripp et al. 2008). A full accounting of the CGM mass by physical phase is thus an important longterm goal of the COS-Halos survey. Finally, the COS-Halos survey is also motivated by a desire to assess the heavy element content of the CGM and to find out how far from galaxies metals have propagated. Metal transport is important both as a factor in Galactic chemical evolution and as a tracer of feedback by galaxies into their surroundings, which in turn alters their evolution.

Papers addressing the main COS-Halos survey so far include the Werk et al. (2012) compilation of galaxy spectroscopy, the Tumlinson et al. (2011a) study of O vi bimodality in galaxy halos, the Thom et al. (2012) study of $\mathrm{H}_{\mathrm{I}}$ in early type galaxies, and the Werk et al. (2013) empirical description of the CGM as seen in metal lines. These studies collectively show the power of the COS-Halos dataset to reveal the properties of gas surrounding galaxies and its relationship to the properties of those galaxies. The results of these main survey papers will be summarized in connection with results below.

Data from COS-Halos has also been used for several additional investigations of gas within and around galaxies in the low-redshift universe, apart from the main survey. Tumlinson et al. (2011b) reported the detection of a strong intervening O VI absorber associated with a galaxy toward J1009+0713, and found it to be a complex, multiphase system associated with a nearby star-forming galaxy that likely contributes to the ionization of the detected absorption. Thom et al. (2011) reported the detection of a metal-poor cloud in close association with a nearby star-forming galaxy that resembles the expected properties of cold, metal-poor accretion onto star-forming galaxies. Meiring et al. (2011) presented the first survey of low-redshift damped Ly $\alpha$ systems (DLAs) using COS, which were analyzed 
for metallicities and relative abundances by Battisti et al. (2012). Lehner et al. (2012) combined a portion of data from COS-Halos with other COS programs to produce an unbiased estimate of the covering fraction of high-velocity ionized gas in the MW. Finally, using data from a combination of COS programs with published absorbers, Lehner et al. (2013) have investigated the bimodal distribution of metallicities in Lyman-limit systems (LLSs) presumably tracing CGM gas.

This paper presents both the general design and execution of COS-Halos and the resulting census of $\mathrm{H}$ i detected near the targeted galaxies. The paper is organized as follows. Section 2 describes the design features of the program and how they meet the scientific goals described in this introduction. Section 3 covers the data collection and analysis, concentrating on the COS data; the reader interested in the full details of the complementary ground-based spectroscopic survey is referred to Werk et al. (2012). Section 4 presents the basic empirical characterization of the H I near the survey galaxies, in terms of absorption strength, kinematics, and correlations with galaxy properties. Section 5 compares these results to prior studies of H I near galaxies. Section 6 examines possible origins for the detected $\mathrm{HI}$, including gas inside the targeted halos and gas outside in the IGM, and various specific sites of origin such as satellite galaxies or galaxy groups. Section 7 summarizes our major conclusions.

Throughout our analysis we adopted a cosmology specified by WMAP3 $\left(\Omega_{m}=0.238, \Omega_{\Lambda}=0.762, H_{0}=73.2 \mathrm{~km} \mathrm{~s}^{-1} \mathrm{Mpc}^{-1}\right.$, $\left.\Omega_{b}=0.0416\right)$. Distances and galaxy virial radii are given in proper coordinates.

\section{THE DESIGN OF COS-HALOS}

\subsection{QSO and Galaxy Selection}

We began our selection with the Sloan Digital Sky Survey (SDSS) DR5 catalog of quasars (Schneider et al. 2007). This catalog was cross-matched with the Galaxy Evolution Explorer $(G A L E X)$ GR3 photometric catalog ${ }^{12}$ to assign FUV and NUV magnitudes to each SDSS QSO. Objects with multi-epoch detections in the GALEX data had their fluxes averaged, weighted by their respective errors, for a final value. We selected for QSOs with $z_{\mathrm{em}}<1$ to minimize contamination by foreground LLSs that would have masked absorption by the targeted galaxies at $z \lesssim 0.3$. We included in our search only QSOs bright enough $(\widetilde{G A L E X} \mathrm{FUV} \lesssim 18.5)$ for COS to obtain $\mathrm{S} / \mathrm{N} \sim 10-12$ in five orbits with the medium-resolution gratings. We also avoided QSOs with $>1 \AA \mathrm{Mg}$ II absorbers at $z>0.4$ based on published catalogs (Prochter et al. 2006) and visual inspection of optical QSO spectra. This was done to avoid losing a large range of QSO spectrum to LLSs. This criterion selects against absorber systems at $z \gtrsim 0.4$, well above the redshifts of interest for the prime sample. This screening benefits our own scientific goals, but we emphasize that the COS-Halos dataset is strongly biased against any absorbers that would exhibit strong $\mathrm{Mg}$ II at $z \gtrsim 0.4$, such as metal-enriched LLSs and DLAs, and therefore should not be used to derive quantities such as redshift number-density $(d N / d z)$ where unbiased sightlines are necessary. We did not apply any other selection criteria to the QSOs themselves. The final list of QSOs observed appears in Table 1.

\subsection{Galaxy Catalog and Selection}

COS-Halos departs from the typical selection technique used for QSO absorption-line studies by choosing galaxies in

\footnotetext{
$\overline{12 \text { Martin et al. (2005), http://galex.stsci.edu }}$
}

advance of absorbers but without secure spectroscopic redshifts. Most previous studies have either selected galaxies at known (usually very low) redshifts (e.g., Bowen et al. 1996), obtained spectroscopic redshifts prior to analyzing absorber data (e.g., Prochaska et al. 2011a), performed post-facto galaxy surveys after the absorber data were obtained (e.g., Chen et al. 2001; Tripp et al. 1998), or compiled pre-existing galaxy catalogs surrounding the QSOs for which absorption data had already been obtained or was expected to be obtained (e.g., Penton et al. 2002; Wakker \& Savage 2009). Rather than adopt any of these techniques, COS-Halos chose galaxies in advance, but based on SDSS photometric redshifts. Selection based on photometric redshifts vastly increases the number of galaxies available, since the photometric survey (DR5 at the time of our planning in 2008) contained tens of millions of galaxies but only 675,000 of these were observed spectroscopically. Furthermore the SDSS spectroscopic survey is concentrated at $z \leqslant 0.1$, too low to place O VI in the $\operatorname{COS}$ band $(\lambda>1150 \AA)$. Using the photometric redshifts allows a selection to push beyond $z \sim 0.1$, and makes close pairings between galaxies and QSOs much easier to find, especially at the low projected angular separations needed to probe the CGM inside $\sim 50 \mathrm{kpc}$. The tradeoff is that secure spectroscopic redshifts must be obtained subsequently. However, this places COS-Halos in no worse position than every survey that relies on post-facto galaxy surveys with no prior knowledge of galaxies in the field. With relative errors of $\sigma_{z} / z \lesssim 0.2$ (Oyaizu et al. 2008), the photometric redshifts were adequate to ensure that the key ionization diagnostics of CGM gas, particularly H I and O VI, still fall within the 1150-1800 range of the COS $\mathrm{M}$ gratings. Thus the photometric redshifts allowed us to select good candidate pairs from a large pool with little additional risk.

We began our galaxy searches by obtaining a list of all SDSS galaxies (photoObj.type $=3$ ) within $8^{\prime}(\simeq 1 \mathrm{Mpc}$ at $z=0.1)$ of every QSO that met the FUV magnitude and redshift selection criteria described above. The dereddened ugriz magnitudes of these galaxies were then $k$-corrected (Blanton \& Roweis 2007) based on the photometric redshift $\left(z_{3}\right.$, which agreed best with the spectroscopic redshifts when both were available). We adopted a cosmology specified by $\operatorname{WMAP3}\left(\Omega_{m}=0.238, \Omega_{\Lambda}=\right.$ $\left.0.762, H_{0}=73.2 \mathrm{~km} \mathrm{~s}^{-1} \mathrm{Mpc}^{-1}, \Omega_{b}=0.0416\right)$. From these $k$-corrected magnitudes we derived estimated stellar masses using the relation of McIntosh et al. (2008). Galaxy impact parameters to the sightline were computed in physical kpc at $z_{3}$ in the adopted cosmology. All these galaxy quantities entered our master catalog of galaxies from which to choose pairings with QSOs.

We then binned this subset of galaxies by mass over $\log M_{*} \simeq$ $10-11$ and by impact parameter over $\rho<150 \mathrm{kpc}$, in 25 bins of $\Delta M_{*}=0.2$ and $\Delta \rho=30 \mathrm{kpc}$ (see Figure 1). At the time of selection, these quantities were estimated based on photometric redshifts (Figure 1 adopts the final spectroscopic redshifts). We required that the galaxies satisfy the condition $0.11+\sigma_{z}<z_{3}<z_{\mathrm{QSO}}-\sigma_{z}$ to ensure that $\mathrm{O}$ vI was redshifted into the COS FUV band, and to reduce the chance that the galaxy would turn out to lie at the same redshift as the QSO. We attempted to place two galaxies in each bin, to cover this parameter space as evenly as possible. All else being equal, we chose pairings with brighter QSOs to minimize the observing time required to build a sample. This led to a selected sample of 43 "target" galaxies, which was then modified by additions and subtractions (see below) into a sample of the 44 galaxies shown in Figures 2 and 3 and listed in the first part of Table 2. The 
Table 1

COS-Halos QSO Sample

\begin{tabular}{|c|c|c|c|c|c|c|c|}
\hline QSO Name & $\alpha(\mathrm{J} 2000)$ & $\delta(\mathrm{J} 2000)$ & $z_{\mathrm{em}}$ & $m_{\mathrm{FUV}}$ & $\begin{array}{c}t_{\mathrm{G} 130 \mathrm{M}} \\
\text { (s) }\end{array}$ & $\begin{array}{c}t_{\mathrm{G} 160 \mathrm{M}} \\
\text { (s) }\end{array}$ & Visit $^{\mathrm{a}}$ \\
\hline SDSS J004222.29-103743.8 & 10.593 & -10.629 & 0.424 & 17.42 & 2448 & 2781 & 05 \\
\hline SDSS J022614.46+001529.7 & 36.560 & 0.258 & 0.615 & 17.87 & 5826 & 4361 & 26 A5 \\
\hline SDSS J040148.98-054056.5 & 60.454 & -5.682 & 0.570 & 18.33 & 5377 & 5912 & 22 \\
\hline SDSS J080359.23+433258.4 & 120.997 & 43.550 & 0.449 & 18.41 & 5207 & 6110 & 09 \\
\hline SDSS J082024.21+233450.4 & 125.101 & 23.581 & 0.470 & 18.37 & 5035 & 5926 & 17 \\
\hline SDSS J091029.75+101413.6 & 137.624 & 10.237 & 0.463 & 18.72 & 4913 & 8699 & 18 \\
\hline SDSS J091440.38+282330.6 & 138.668 & 28.392 & 0.735 & 18.76 & 5093 & 8735 & 21 \\
\hline SDSS J092554.70+400414.1 & 141.478 & 40.071 & 0.471 & 18.14 & 3765 & 4303 & 24 \\
\hline SDSS J092837.98+602521.0 & 142.158 & 60.423 & 0.296 & 17.59 & 2311 & 3052 & 25 \\
\hline SDSS J093518.19+020415.5 & 143.826 & 2.071 & 0.649 & 18.24 & 3625 & 4018 & 43 \\
\hline SDSS J094331.61+053131.4 & 145.882 & 5.525 & 0.564 & 18.21 & 3662 & 3945 & 42 \\
\hline SDSS J095000.73+483129.3 & 147.503 & 48.525 & 0.589 & 17.86 & 2445 & 2927 & 27 \\
\hline SDSS J100902.06+071343.8 & 152.259 & 7.229 & 0.456 & 18.09 & 3688 & 4009 & 1344 \\
\hline SDSS J101622.60+470643.3 & 154.094 & 47.112 & 0.822 & 17.99 & 2416 & 2927 & 07 \\
\hline SDSS J102218.99+013218.8 & 155.579 & 1.539 & 0.789 & 17.92 & 2297 & 2770 & 29 \\
\hline SDSS J111239.11+353928.2 & 168.163 & 35.658 & 0.636 & 18.36 & 5439 & 6030 & 16 \\
\hline SDSS J113327.78+032719.1 & 173.366 & 3.455 & 0.525 & 18.58 & 4993 & 5896 & 01 \\
\hline SDSS J115758.72-002220.8 & 179.495 & -0.372 & 0.260 & 17.74 & 3417 & 4252 & 03 \\
\hline SDSS J122035.10+385316.4 & 185.146 & 38.888 & 0.376 & 17.83 & 2364 & 2837 & 41 \\
\hline SDSS J123304.05-003134.1 & 188.267 & -0.526 & 0.471 & 18.30 & 5305 & 5896 & 15 \\
\hline SDSS J123335.07+475800.4 & 188.396 & 47.967 & 0.382 & 18.02 & 3885 & 4217 & 31 \\
\hline SDSS J124154.02+572107.3 & 190.475 & 57.352 & 0.583 & 18.56 & 5839 & 9497 & 06 \\
\hline SDSS J124511.25+335610.1 & 191.297 & 33.936 & 0.711 & 18.43 & 3824 & 6929 & 36 \\
\hline SDSS J132222.68+464535.2 & 200.594 & 46.760 & 0.375 & 18.02 & 3902 & 4248 & 11 \\
\hline SDSS J133045.15+281321.4 & 202.688 & 28.223 & 0.417 & 18.32 & 5351 & 5942 & 32 \\
\hline SDSS J134251.60-005345.3 & 205.715 & -0.896 & 0.326 & 17.42 & 2297 & 2770 & 39 \\
\hline SDSS J141910.20+420746.9 & 214.793 & 42.130 & 0.873 & 17.83 & 3676 & 4354 & 23 \\
\hline SDSS J143511.53+360437.2 & 218.798 & 36.077 & 0.429 & 17.83 & 3466 & 4424 & 12 \\
\hline SDSS J143726.14+504555.8 & 219.359 & 50.766 & 0.783 & 18.83 & 5421 & 9299 & 38 \\
\hline SDSS J144511.28+342825.4 & 221.297 & 34.474 & 0.697 & 18.49 & 6547 & 7371 & 40 \\
\hline SDSS J151428.64+361957.9 & 228.619 & 36.333 & 0.695 & 18.53 & 10446 & 12060 & 14 A4 \\
\hline SDSS J155048.29+400144.9 & 237.701 & 40.029 & 0.497 & 18.00 & 3804 & 4160 & 35 \\
\hline SDSS J155304.92+354828.6 & 238.271 & 35.808 & 0.722 & 17.66 & 4228 & 5674 & 33 A3 \\
\hline SDSS J155504.39+362848.0 & 238.768 & 36.480 & 0.714 & 18.45 & 5151 & 6030 & 08 \\
\hline SDSS J161649.42+415416.3 & 244.206 & 41.905 & 0.440 & 17.59 & 3644 & 4604 & 28 \\
\hline SDSS J161711.42+063833.4 & 244.298 & 6.643 & 0.229 & 17.86 & 3596 & 4128 & 04 \\
\hline SDSS J161916.54+334238.4 & 244.819 & 33.711 & 0.471 & 18.57 & 5347 & 8789 & 30 \\
\hline SDSS J225738.20+134045.4 & 344.409 & 13.679 & 0.594 & 17.92 & 3428 & 4274 & 34 \\
\hline SDSS J234500.43-005936.0 & 356.252 & -0.993 & 0.789 & 18.43 & 5125 & 5896 & 10 \\
\hline
\end{tabular}

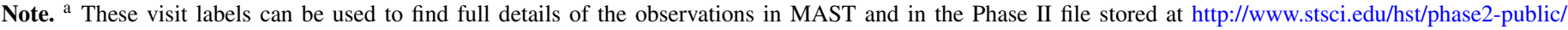
11598.apt.

technique of building an aggregate map of galaxies, each probed by a single sightline, is visualized in Figure 2, which shifts the target galaxy to the center and displays the QSOs distributed properly in impact parameter and position angle. Figure 3 shows the galaxies in a color-magnitude diagram along with the parent galaxy population from the NYU-VAGC (Blanton et al. 2005).

\subsection{Target and Bonus Galaxies}

While collecting spectra on the "target" galaxies-those that were originally selected-we also obtained spectra on photometrically selected galaxies near the sightline that met the criteria of stellar mass and impact parameter but different $z_{3}$. Galaxies that ended up with $z \simeq z_{\text {QSO }}$ were discarded, but those with acceptable redshifts are included in our analysis as "bonus" galaxies. Note that these galaxies were included without regard to absorption along the sightline, as generally their redshifts were obtained before the COS data, so they fulfill the same requirements of prior galaxy selection as the original "targets." The galaxies in our sample are detailed in Table 2. The listed properties are drawn from Werk et al. (2012) directly and are listed here for reference in later tables.

\subsection{Galaxies Omitted from the Main Sample}

Four of the galaxies originally targeted turned out to have spectroscopic redshifts of $z \lesssim 0.1$, placing their $\mathrm{O}$ VI out of the COS band. In these four cases the galaxies also have significantly lower luminosity and/or stellar mass than we intended to include in the sample. These sub- $L^{*}$ galaxies have been excised from the main sample that is analyzed further here. Another originally selected galaxy (J1553+3548 97_30) has not had its spectroscopic redshift measured securely, so we cannot include it in the sample. These five cases are listed in Table 2 for completeness.

\subsection{Galaxy Neighbors and Environment}

The properties of gas in galaxy halos, and even the galaxies themselves, can be influenced by galaxies nearby in group 


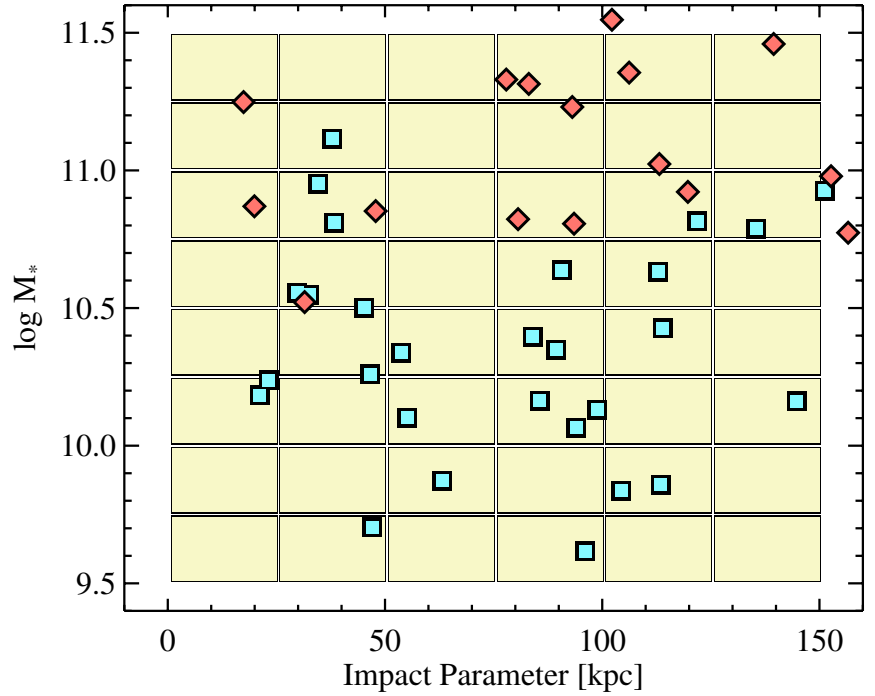

Figure 1. Original COS-Halos selection distributed 43 galaxies in 25 bins of stellar mass and impact parameter between $\log M_{*} / M_{\odot}=10$ and 11 as evenly as possible. The confirmed spectroscopic redshifts, as well as target substitutions and bonus galaxies, brought the sample to those 44 galaxies shown, which populate the 48 bins in this parameter space as shown. The stellar masses assume a Salpeter IMF, and the impact parameters are given in physical kpc at the galaxy spectroscopic redshift $z_{\text {sys }}$. The symbol coding of blue squares for star-forming galaxies and red diamonds for passive galaxies are used throughout all subsequent figures.

(A color version of this figure is available in the online journal.)

environments or the same regions of the large-scale structure (LSS). Thus quantifying galaxy environment can be an important component of studies of their star formation, evolution, and surrounding gas.

The COS-Halos galaxies were selected based on SDSS photometric redshifts, so this survey does not feature the ready recovery of environment information enjoyed by surveys done with multi-object spectroscopy before or after the absorptionline data is obtained. However, to mitigate the effects of multiple galaxies at the same redshift, during the selection of targeted galaxies we preferred candidate galaxies without photometric redshift coincidences within the same $\sim 1$ Mpc search radius used to pick the candidate targets. That is, sightlines with

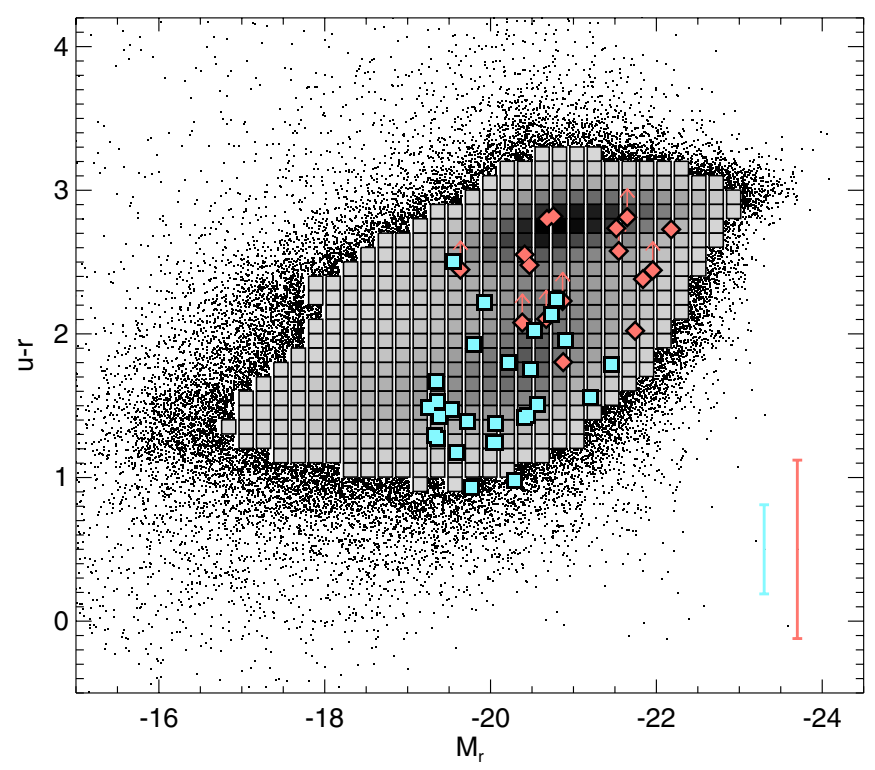

Figure 3. COS-Halos sample in a color-magnitude diagram using the $u-r$ color and the absolute $r$-band magnitude, both from dereddened, $k$-corrected SDSS photometry. Color coding is the same as before. The background distribution of galaxy number densities is derived from the NYU Value Added Galaxy Catalog (Blanton et al. 2005). The bars at lower left show the mean error in the colors for the star-forming $( \pm 0.3 \mathrm{mag})$ and passive subsamples $( \pm 0.6 \mathrm{mag})$. Roughly half of the passive galaxies have uncertain intrinsic colors with errors up to 1-2 mag as a consequence of their non-detection in the SDSS $u$ band; these are plotted with lower limit arrows.

(A color version of this figure is available in the online journal.)

two photo- $z$ candidates at similar $z$ (within about $1 \sigma_{z}$ ) were not chosen. This choice introduces a bias into the nearby environment of these galaxies, in favor of isolation over group membership with other galaxies. This bias acts mainly against other $\sim L^{*}$ or brighter galaxies, as fainter galaxies at the same redshifts either have much larger photo- $z$ errors or drop out of the SDSS photometry altogether. This bias against close neighbors is difficult to quantify because it can only really be assessed with a complete spectroscopic redshift survey and comparisons to control samples to which different selection criteria have been applied. However, because we did not aggressively omit all possible coincidences and photometric redshift errors are not

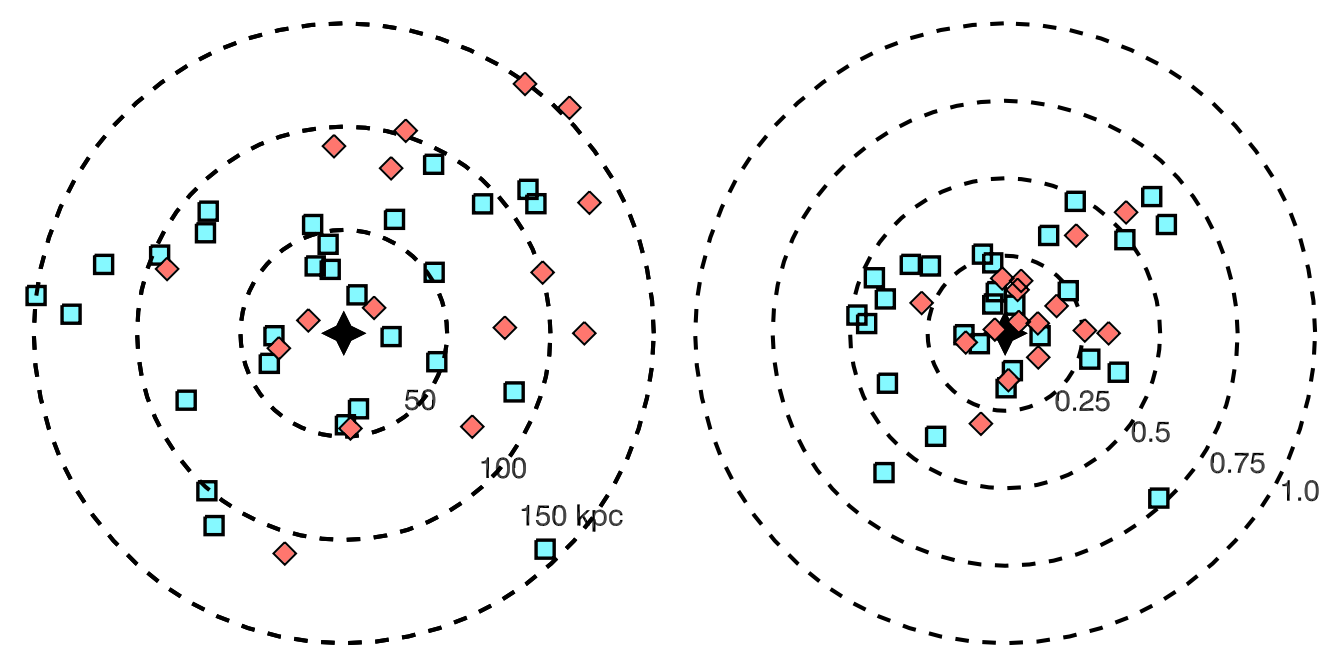

Figure 2. Target figure showing the distribution of QSOs on the sky with respect to the target galaxies (shifted to the center). Star-forming galaxies are shown in blue, and passively evolving galaxies in red. At left the radial coordinate is physical kpc at the galaxy redshift, at right this coordinate is translated to the fraction of galaxy's virial radius, $R_{\mathrm{vir}}$, at which the sightline intercepts the halo. No knowledge of galaxy disk orientation or inclination with respect to the sightline is implied here.

(A color version of this figure is available in the online journal.) 
Table 2

COS-Halos Galaxy Sample

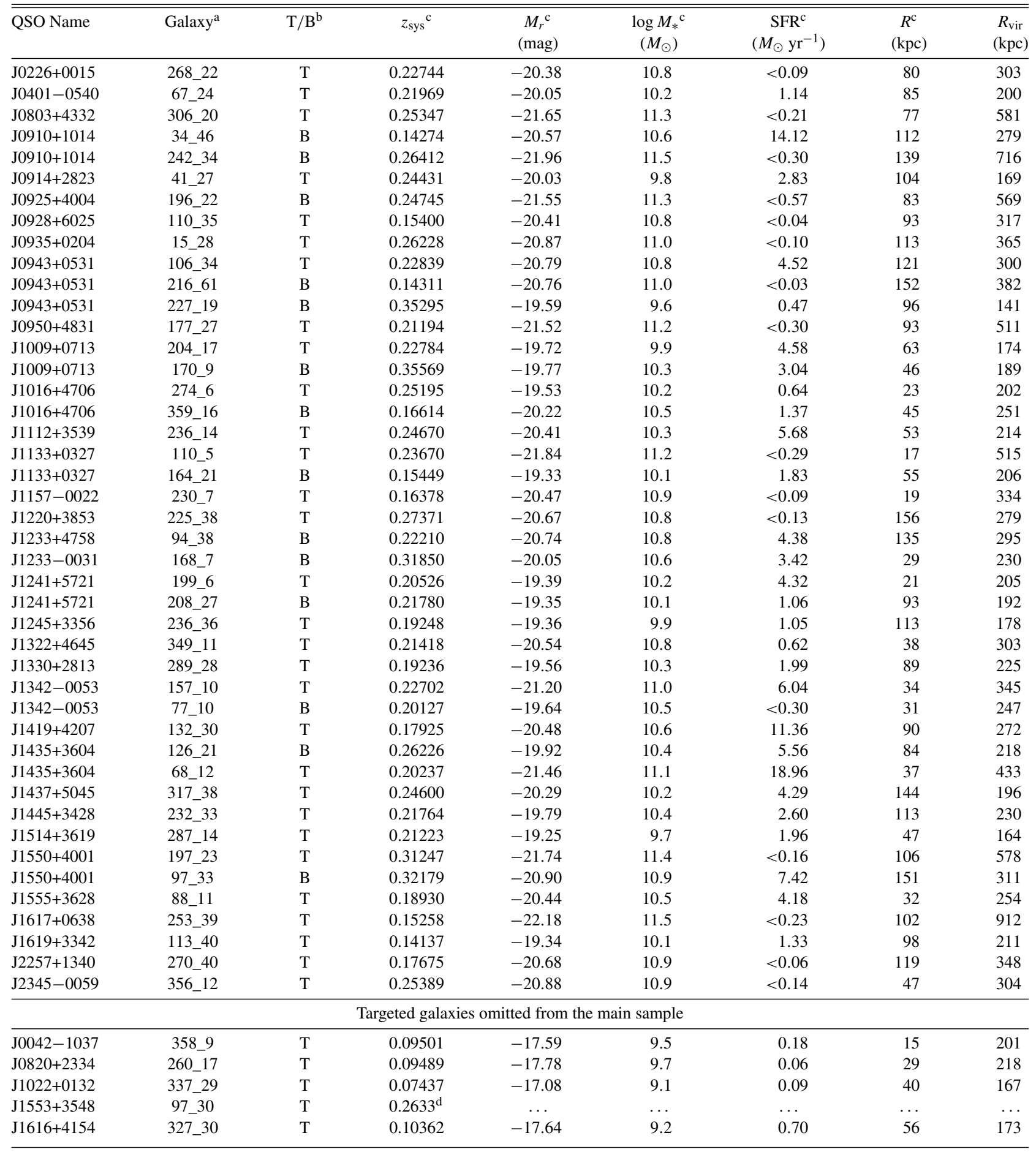

Notes.

${ }^{a}$ We label the galaxies by the position angle with respect to the QSO, $\mathrm{N}$ through $\mathrm{E}$, and with the angular separation in arcsec.

${ }^{\mathrm{b}}$ Indicates whether the galaxy was a pre-selected target (T) or a bonus galaxy (B) meeting the same criteria.

${ }^{\mathrm{c}}$ Galaxy redshift $z_{\text {sys }}$, absolute magnitude $M_{r}$, stellar mass $M_{*}$, star formation rate (SFR), and impact parameter $R$ are drawn from Werk et al. (2012).

d Photometric redshift. This galaxy does not have a secure spectroscopic redshift.

perfectly well-behaved, and because we did not apply any such screening to the bonus galaxies, redshift coincidences did arise during the spectroscopic follow-up stage of the survey.

The full list of galaxies for which we obtained spectroscopy is described in Werk et al. (2012). In a few fields the follow-up spectroscopy identified more than one galaxy at the redshift of the pre-selected, targeted galaxy. In these cases, we have taken the most massive (equivalently the brightest in SDSS $r$ ) as the adopted "canonical" galaxy for analysis purposes. There are only two cases for which choosing the closest galaxy rather than the most massive would change the type of the galaxy and therefore affect the comparisons we do in later 
sections. The galaxy $270 \_40$ toward $\mathbf{J} 2257+1340$ is a passive galaxy with two smaller, star-forming galaxies slightly nearer the sightline. This system is not detected in H I, so the change would slightly affect the $\mathrm{H}$ I detection rates examined below (see Section 4.1). The damped system associated with galaxy 110_35 toward J0928+6025 has a less massive star-forming galaxy nearer the sightline, which would result in two, rather than just one, of the three damped systems being associated with star-forming galaxies. These minor ambiguities do not affect the larger conclusions reached below.

We have not yet performed complete spectroscopic surveys in these fields, so we cannot say anything more about their large-scale environment, near neighbors, or possible satellites without knowledge of exact redshifts. To address this issue with the available information, Werk et al. (2012) searched the fields surrounding the targeted galaxies for photometric redshift candidates and then quantified neighboring galaxies by (1) counting the number of galaxies within $5 \mathrm{Mpc}$, and (2) identifying the distance to the nearest neighbor. This same search was performed for a set of 500 SDSS control galaxies with the same range of $r$-band absolute magnitudes and redshift as COS-Halos. Figure 8 of Werk et al. (2012) shows the results of these environment tests. For all but a few COS-Halos galaxies the nearest-neighbor candidate at similar luminosity is at $>1 \mathrm{Mpc}$ projected separation, and the median nearestneighbor distance is $2.5 \mathrm{Mpc}(2.7 \mathrm{Mpc}$ in the control sample, a statistically insignificant difference). There is also no significant difference in the counts of galaxies within $5 \mathrm{Mpc}$ for COSHalos and the control sample. On this basis, Werk et al. (2012) concluded that there was no evidence that the COSHalos galaxies are unusual in their large-scale $(1-5 \mathrm{Mpc})$ environments. The canonical galaxy in each field is the most luminous galaxy within $300 \mathrm{kpc}$ of the QSO sightline at its redshift and in most cases there are no likely $L \sim L^{*}$ photo- $z$ candidates within $1 \mathrm{Mpc}$. We also believe that the large-scale environments $(\lesssim 5 \mathrm{Mpc})$ of these galaxies are not unusual. We will revisit this issue when considering a group gas origin for the detected halo gas (Section 6.2.2).

\section{DATA COLLECTION AND ANALYSIS}

\subsection{COS Data Reduction}

The COS observations are detailed in Table 1. We planned for a uniform set of COS data obtained with both of the FUV medium-resolution gratings, G130M and G160M (Dixon 2010; Green et al. 2012). We used the FUV channel detectors in TAGFLASH mode. We tuned the central wavelengths (CENWAVEs) to avoid placing the $14 \AA$ gap between FUV detector segments at the position of $\mathrm{O}$ VI for the targeted galaxies, based on $z_{\text {sys }}$, and to ensure that $\mathrm{O}$ vi would be covered at the short-wavelength end of the G130M setting. Generally two CENWAVEs were used to ensure complete wavelength coverage from 1140-1800 , though for a few QSOs only one position was used to ensure that all the exposure time went into covering lines expected to be near the edge of the recorded spectrum. The balance between the time allocated to G130M and G160M was set to ensure $\mathrm{S} / \mathrm{N}=8$ per resolution element or better over 1150-1700 ̊.

The COS data were obtained from MAST ${ }^{13}$ and uniformly processed by CALCOS (v2.12) with standard parameters and reference files. We performed our own co-additions to merge exposures obtained with different CENWAVEs and the two gratings. This was the same method used by Meiring et al. (2011),

\footnotetext{
13 http://archive.stsci.edu
}

Tumlinson et al. (2011b) and Thom et al. (2011). This procedure operates on the gross counts vectors stored in the CALCOS $\mathrm{x} 1 \mathrm{~d}$ output files, and tracks the count rates in each raw pixel; each COS resolution element at $R \sim 18,000$ covers $\simeq 16 \mathrm{~km} \mathrm{~s}^{-1}$ and is sampled by six raw pixels. We track counts and count rates so that variances can be computed rigorously in the Poisson limit at low count rates using the tables of Gehrels (1986). Exposures taken at the same grating and CENWAVE were added first. This coadd was then summed with exposures in the same grating at different CENWAVEs, followed by a sum of the two grating spectra to produce a single one-dimensional (1D) trace from 1150-1800 A. At each stage of the co-addition, exposures were shifted in wavelength by steps of 1 raw pixel (1/6 resel) by aligning common MW interstellar lines (e.g., Si II, C II, Al II) in velocity space. These alignments ensure that small zeropoint shifts in the wavelength solution from exposure to exposure are mitigated as much as possible.

The photocathode grid wires lying above the COS microchannel plates cast shadows onto the detector and are the main source of fixed-pattern noise in our data. Smaller fluctuations caused by the microchannel plate pores generally do not appear at the signal-to-noise ratios $(\mathrm{S} / \mathrm{Ns})$ of our data. However, the grid wires are easily visible in our raw data and must be corrected. There are other fixed-pattern noise features that must also be removed. We adopted flat-field reference files prepared and communicated to us by D. Massa at STScI and filtered for high-frequency noise by E. Jenkins. These 1D files allow us to correct the shadowed pixels by modifying the effective exposure time and count rate in each pixel prior to coadding it with the others. The resulting 1D, flat-corrected summed spectra were then binned by 3 raw pixels to give final science-grade spectra with $\sim 2$ bins and $\mathrm{S} / \mathrm{N} \sim 10-12$ per COS resolution element $\left(\mathrm{FWHM} \simeq 18 \mathrm{~km} \mathrm{~s}^{-1}\right)$. These 2-bins-per-resel spectra are in units of wavelength and counts per second and are used in all further analysis.

\subsection{COS Data Analysis}

Our absorption-line analysis begins with the optimally binned 1D count-rate spectra described above. The goal of the absorption-line analysis procedure is to identify and measure a set of common lines associated with the sample galaxies. The design of COS-Halos introduces a significant simplification over the usual requirement of identifying every line in a QSO spectrum, because we have measured the galaxy systematic redshifts $z_{\text {sys }}$ to high precision $\left(\sigma_{\text {specz }} \sim 30 \mathrm{~km} \mathrm{~s}^{-1}\right.$ in the rest-frame $)$ and can focus on common lines at predictable places in observed wavelength. A subset of the common lineset appears in Figure 4 to illustrate the redshift ranges over which they are available in the COS-Halos data, including the optical data described further in Section 3.4. This figure also motivates the design choice to focus on galaxies at $z \simeq 0.1-0.3$. Of course the availability of a given line depends not only on redshift but on local $\mathrm{S} / \mathrm{N}$ and the contaminating presence of other lines, so that each line in the common set must be identified and evaluated on its own to judge detection.

We developed a semi-automated procedure for identifying and measuring lines that automatically extracts and processes slices of spectrum around each of the lines in the standard set. This procedure is used here and in the complementary analysis of metal lines in COS-Halos (Werk et al. 2013). Each slice covers \pm 500 bins (each bin is 3 raw pixels spanning approximately $1 / 2$ of a resolution element) on either side of the systemic galaxy redshift, whether the corresponding line is detected or not. These 


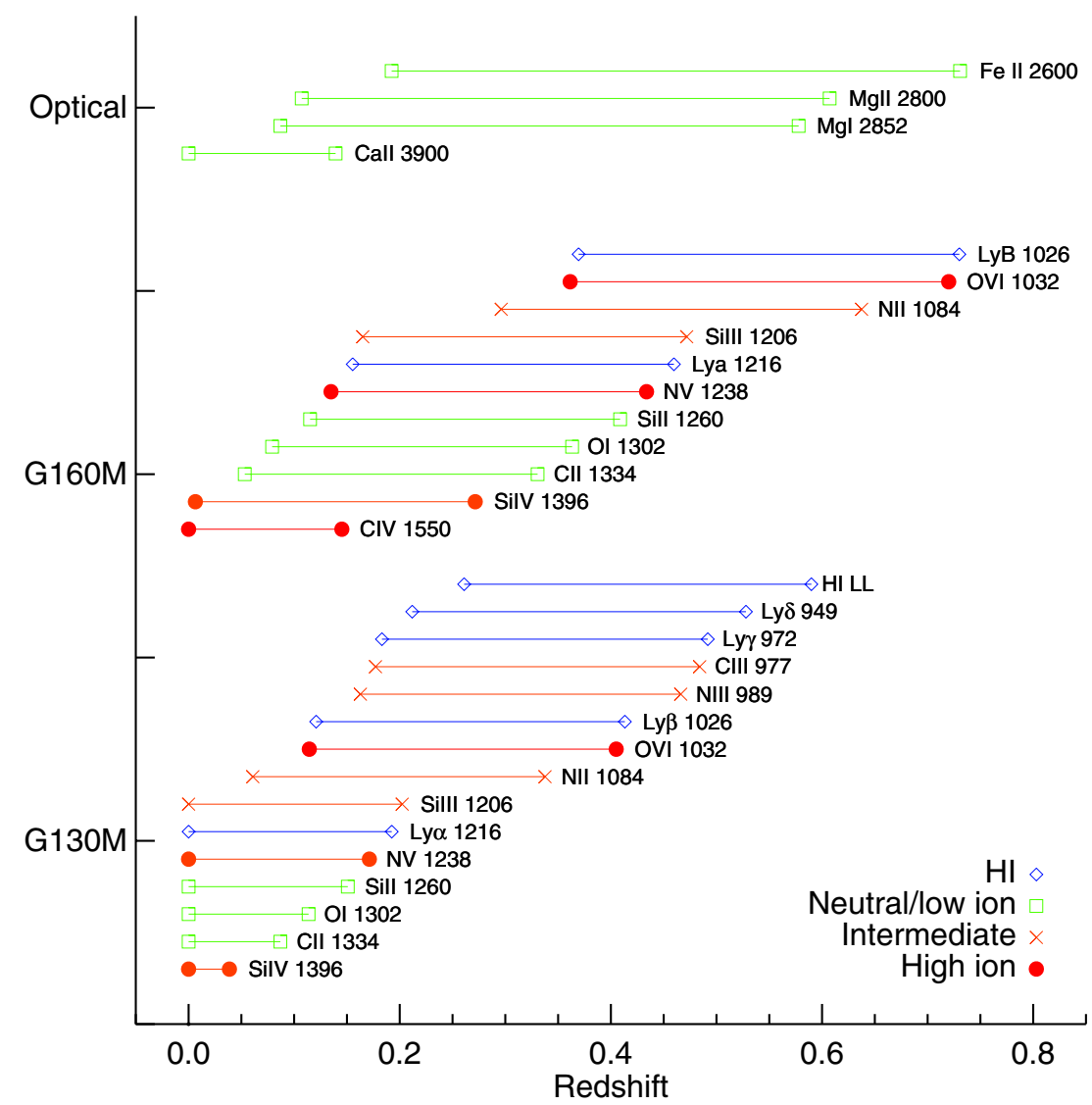

Figure 4. COS-Halos was designed with galaxies at $z=0.15-0.35$ to place O VI and other key ionization diagnostics on the COS FUV G130M and G160M gratings. (A color version of this figure is available in the online journal.)

slices are then independently continuum-normalized using fifthorder Legendre polynomials and trimmed to $\pm 1500 \mathrm{~km} \mathrm{~s}^{-1}$ of $v=0$. Lines of a single element are grouped and plotted together in rest-frame velocity space, as shown in the example in Figure 5. The complete set of stack plots is available as Figure set 5 in the online journal.

These uniform plot sets allow for quick assessments of which lines from a given element and species should be measured. These visual inspections determine only whether or not a particular line will be flagged for measurement; they do not decide formal detections or limits. They also rely on "sightline" linelists, or catalogs of absorption lines from systems at other redshifts in the sightline, to facilitate the identification of nearby lines blended with absorption from the target. The process is then repeated to measure equivalent widths and apparent column densities over velocity intervals specified manually in the system linelist by an inspection of the stack plots. This generates rest-frame equivalent widths $W_{r}$ for each line, and assigns data quality flags based on $\mathrm{S} / \mathrm{N}$, blending, and saturation. We calculated apparent column densities using the method of Savage \& Sembach (1991), which converts the normalized flux spectrum into an optical depth and then to an apparent column density prior to integrating over the desired velocity range. Detections are automatically flagged as such by a requirement for $\geqslant 3 \sigma$ significance (not the visual inspection used to flag the line for measurement). Undetected lines are recorded as upper limits based on the local $\mathrm{S} / \mathrm{N}$, usually integrated over $\pm 50 \mathrm{~km} \mathrm{~s}^{-1}$; the errors and limits in the stack plots and Tables 4 and 5 are $1 \sigma$ values, and we generally adopt $2 \sigma$ limits in analysis and interpretive plots. Any line that drops below $10 \%$ transmission relative to the normalized continuum is automatically flagged as saturated and treated as a lower limit for column density measurement; lines can also be manually flagged as saturated based on more subjective judgement, flagged as blended based on detailed inspection of possible contamination, or left out entirely if the data quality is poor, continuum normalization is inadequate, or for any other reason prohibiting a clean measurement. Even lines that are not used generally appear in the stack plots for completeness. The directly integrated apparent column densities (replaced with the Ly $\alpha$ profile fitting for damped systems) are used in almost all of our subsequent analysis. Tests performed with the profile-fitted column densities are called out as such throughout the text.

After this process, the resulting rest-frame equivalent width $W_{r}$, directly integrated column density $N$, velocity ranges, and data quality flags are stored in a table of line results for each system for later analysis. Adopted column densities are computed using an average of uncontaminated, unsaturated Lyman lines weighed by their relative errors. For systems in which all the detected lines are saturated, the adopted column density is a lower limit set by the measurement for the weakest (e.g., most sensitive) available line. At the end of the process the plots are then regenerated to incorporate all the resulting information about column densities, errors, flags, and profile fits as shown in Figure 5.

Our automated analysis routines searched for every $\mathrm{H}$ I Lyman series line that was covered in the COS data. The number of available lines increases as the galaxy redshift increases and shorter-wavelength Lyman lines redshift onto the detector. Ly $\alpha$ always falls on the detector, but in a few cases is blended 
Table 3

COS-Halos O vi Sample Error Budgets

\begin{tabular}{|c|c|c|c|c|c|}
\hline Category & Ref No. & Quantity & Statistical & Systematic & Comments \\
\hline \multirow[t]{7}{*}{ Absorbers } & 1 & $v_{\text {sys }}$ zeropoint & $\ldots$ & $\pm 25 \mathrm{~km} \mathrm{~s}^{-1}$ & Directly from $z_{\text {sys }}$ of target galaxy \\
\hline & 2 & " & $\ldots$ & $\pm 2-3$ pix $=6-8 \mathrm{~km} \mathrm{~s}^{-1}$ & $\operatorname{COS} \lambda$ zeropoint $^{\mathrm{a}}$ \\
\hline & 3 & " & $\ldots$ & $\pm 10 \mathrm{~km} \mathrm{~s}^{-1}$ & Derived from doublet shifts ${ }^{b}$ \\
\hline & 4 & $v_{c}$ total & $\ldots$ & $\pm 30 \mathrm{~km} \mathrm{~s}^{-1}$ & Root-square-sum of (1) and (2)+(3) \\
\hline & 5 & $N$ (integrated) & $0.05-0.2$ dex & $\simeq 0.1 \mathrm{dex}$ & Systematic is continuum \\
\hline & 6 & $N$ (fitted) & $\lesssim 0.2$ dex & Depends on saturation & See Section 3.3 \\
\hline & 7 & $b$ & $\ldots$ & From profile fits & ", \\
\hline \multirow[t]{9}{*}{ Galaxies } & 8 & ugriz & $0.1,0.03,0.03,0.02,0.1$ & $\ldots$ & Medians from SDSS photoObj \\
\hline & 9 & $M_{\text {ugriz }}$ & $\lesssim 0.05$ & $\ldots$ & Propagated from $u g r i z$ and $z_{\text {sys }}$ \\
\hline & 10 & $z_{\text {sys }}$ & $\simeq 0.0002$ & $25 \mathrm{~km} \mathrm{~s}^{-1}$ & Instrumental systematics, Werk et al. (2012) \\
\hline & 11 & $M_{*}$ & Propagated from (8) & 0.2 dex & Bell et al. (2003); McIntosh et al. (2008) \\
\hline & 12 & $M_{\text {halo }}$ & Propagated from (11) & $0.5 \mathrm{dex}$ & Scatter, Moster et al. (2010) \\
\hline & 13 & $R_{\text {vir }}$ & Computed from (12) & 0.15 dex & \\
\hline & 14 & SFR & $\ldots$ & $25 \%$ & Werk et al. (2012) \\
\hline & 15 & {$[\mathrm{O} / \mathrm{H}]$} & $\simeq 0.15 \mathrm{dex}$ & Upper/lower branch & $"$ \\
\hline & 16 & sSFR & $\ldots$ & $50 \%$ & Propagated from SFR and $M_{*}$ \\
\hline
\end{tabular}

Notes.

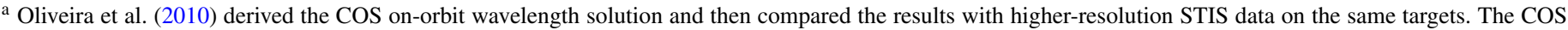
and STIS wavelength zeropoints agree to within 3 raw pixels on average, equivalent to $1 / 2$ COS resolution element.

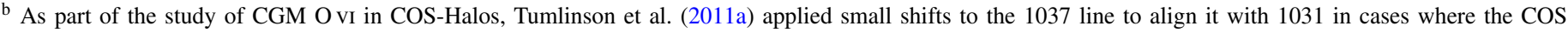

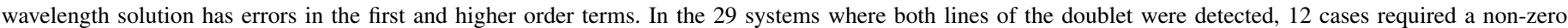

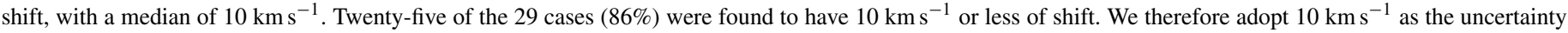

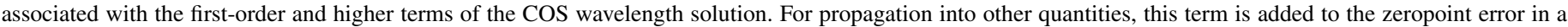
straight sum (terms 2 and 3), and then root-square-summed with (1) to derive the total error in $v_{c}$.

with extraneous absorption and cannot be measured. Errors and systematic uncertainties in our measured quantities are given in Table 3. The basic H I results are listed in Tables 4 and 5, where we list the system labels (= QSO name and galaxy name), the velocity width for integration of the $\operatorname{Ly} \alpha$ profile (or $\operatorname{Ly} \beta$ where Ly $\alpha$ is damped or contaminated). Table 4 gives restframe equivalent widths $W_{r}$ for the first six Lyman series lines. Table 5 gives the full results including line-profile fitting. Full results for all lines are available in our electronic tables and stack plots.

We have attempted to thoroughly identify and measure all absorption appearing within $\pm 600 \mathrm{~km} \mathrm{~s}^{-1}$ of the targeted galaxies, and to associate it convincingly with either the target galaxy itself or an intervening absorber at another redshift. These identifications for unrelated lines are marked in the stack plots. Some weaker lines $(\lesssim 100 \mathrm{~m} \AA)$, particularly those that appear only in the Ly $\alpha$ region of the targeted absorber, cannot be allocated conclusively to intervening systems and cannot be confirmed as Ly $\alpha$ components near the targeted system because they are too weak for $\operatorname{Ly} \beta$ to be expected, or because there is blending, low $\mathrm{S} / \mathrm{N}$, or no wavelength coverage. We have chosen to allocate these lines to the targeted absorber if there is no reliable alternate identification at a different redshift, even if the line cannot be confirmed with $\operatorname{Ly} \beta$; these lines are called out in Table 5 with the flag "Ly $\alpha$ only."

The following analysis uses the rest-frame equivalent widths $W_{r}$ where appropriate, but sometimes also the best estimate or limit on the $\mathrm{H}_{\mathrm{I}}$ column density, $N_{\mathrm{H}}$, derived from fitting the profiles. We obtain the $N_{\mathrm{H} \text { I }}$ measurement or limit with one of three methods depending on the column density of the system expressed in the bitwise flags "Adopt" and "Method" in Table 5. "Adopt" is set to 1 for good measurements of $N_{\mathrm{HI}}, 4$ for nondetections that give upper limits, and 8 for saturated systems that give lower limits. Damped (DLA) or nearly damped (subDLA) absorbers $\left(\log N_{\mathrm{HI}} \simeq 18-20\right)$ have their $N_{\mathrm{H}}$ derived by profile fitting to the Ly $\alpha$ profiles (Adopt $=1$, Method $=3$ ). Undetected absorbers $\left(\log N_{\mathrm{H}_{\mathrm{I}}} \lesssim 14\right.$ ) have their upper limits on $\mathrm{Ly} \alpha$ and $N_{\mathrm{H} \text { I }}$ derived from the directly integrated noise over $\pm 50 \mathrm{~km} \mathrm{~s}^{-1}$ around $z_{\text {sys }}$ (Adopt $=4$, Method $=1$ for direct integration). The plots that follow include these $2 \sigma$ upper limits, while the tables specify the $1 \sigma$ values. Finally, cases with intermediate column densities have their $N_{\mathrm{HI}}$ estimated by direct integration of the line profiles (Method $=1$ ) in apparent column density. If the highest available line is weak and unsaturated, the resulting measurement is considered a measurement (Adopt $=1)$. If the highest available line shows evidence of saturation, the resulting $N_{\mathrm{H}}$ is considered a lower limit (Adopt $=4$ ).

In addition to measurements of line equivalent widths and integrated column densities, for most systems we use lineprofile fitting to resolve the kinematic components of detected absorption and to estimate column densities that take into account profile shapes and saturation. This procedure uses the line slices generated by the automated pipeline and performs Voigt-profile fits to derive the column density $N$, the Doppler width $b$, and the velocity offset $v$ for each component. When multiple transitions for a given species are available, the same $N$, $b$, and $v$ parameters are applied to all transitions and optimized jointly. The number of components fitted to a given profile or set of profiles is determined by visual inspection of the data. We do not attempt to fit every transition of every species and we ignore strong blended profiles. Non-detections are used only when they provide significant constraints on the column density to the high end, such as when a stronger transition is detected and a weaker transition of the same species is not. User judgment is required to decide the number and placement of unrelated nuisance absorption lines from other redshifts that blend with the lines of interest. When multiple transitions of the same ion are fitted simultaneously (such as more than one line from the Lyman series), lines appearing on different regions of the COS detector can experience small shifts (usually $\lesssim 5-10 \mathrm{~km} \mathrm{~s}^{-1}$ but in some 
Table 4

COS-Halos H i Line Measurements

\begin{tabular}{|c|c|c|c|c|c|c|c|c|c|}
\hline QSO Name & Galaxy & $z_{\text {sys }}$ & $\begin{array}{l}v_{\min }, v_{\max }{ }^{\mathrm{a}} \\
\left(\mathrm{km} \mathrm{s}^{-1}\right)\end{array}$ & $\begin{array}{c}W_{r}(\operatorname{Ly} \alpha)^{\mathrm{b}} \\
(\mathrm{m} \AA)\end{array}$ & $\begin{array}{c}W_{r}(\mathrm{Ly} \beta) \\
(\mathrm{m} \AA)\end{array}$ & $\begin{array}{c}W_{r}(\mathrm{Ly} \gamma) \\
(\mathrm{m \AA})\end{array}$ & $\begin{array}{c}W_{r}(\mathrm{Ly} \delta) \\
(\mathrm{m} \AA)\end{array}$ & $\begin{array}{c}W_{r}(\mathrm{Ly} \epsilon) \\
(\mathrm{m} \AA)\end{array}$ & $\begin{array}{c}W_{r}(\mathrm{Ly} \zeta) \\
(\mathrm{m} \AA)\end{array}$ \\
\hline $\mathrm{J} 0226+0015$ & $268 \_22$ & 0.22744 & 0,280 & $486 \pm 20$ & $\ldots$ & $\ldots$ & $\ldots$ & $\ldots$ & $\ldots$ \\
\hline $\mathrm{J} 0401-0540$ & 67_24 & 0.21969 & $-700,120$ & $1234 \pm 34$ & $\ldots$ & $463 \pm 39$ & $290 \pm 25$ & $\ldots$ & $\ldots$ \\
\hline J0803+4332 & 306_20 & 0.25347 & $-250,100$ & $592 \pm 26$ & $277 \pm 18$ & $\ldots$ & $\ldots$ & $\ldots$ & $\ldots$ \\
\hline J0910+1014 & 34_46 & 0.14274 & $-300,300$ & $1444 \pm 48$ & $427 \pm 144$ & $\ldots$ & $\ldots$ & $\ldots$ & $\ldots$ \\
\hline J0910+1014 & 242_34 & 0.26412 & $-300,250$ & $1180 \pm 36$ & $802 \pm 56$ & $278 \pm 70$ & $\ldots$ & $170 \pm 83$ & $101 \pm 83$ \\
\hline J0914+2823 & 41_27 & 0.24431 & $-250,100$ & $731 \pm 21$ & $424 \pm 25$ & $294 \pm 15$ & $228 \pm 17$ & $163 \pm 19$ & $68 \pm 21$ \\
\hline J0925+4004 & 196_22 & 0.24745 & $-75,250$ & $3276 \pm 64$ & $896 \pm 18$ & $\ldots$ & $649 \pm 34$ & $610 \pm 41$ & $\ldots$ \\
\hline J0928+6025 & 110_35 & 0.15400 & $-250,150$ & $2726 \pm 77$ & $828 \pm 47$ & $\ldots$ & $\ldots$ & $\ldots$ & $\ldots$ \\
\hline J0935+0204 & 15_28 & 0.26228 & $-50,50$ & $\leqslant 26$ & $\ldots$ & $\ldots$ & $\ldots$ & $\ldots$ & $\ldots$ \\
\hline J0943+0531 & 106_34 & 0.22839 & 50,330 & $550 \pm 30$ & $\ldots$ & $210 \pm 27$ & $175 \pm 29$ & $\ldots$ & $\ldots$ \\
\hline J0943+0531 & 216_61 & 0.14311 & $-180,50$ & $\ldots$ & $278 \pm 32$ & $\ldots$ & $\ldots$ & $\ldots$ & $\ldots$ \\
\hline J0943+0531 & 227_19 & 0.35295 & $-100,890$ & $2131 \pm 95$ & $1364 \pm 55$ & $953 \pm 41$ & $636 \pm 57$ & $490 \pm 29$ & $\ldots$ \\
\hline J0950+4831 & 177_27 & 0.21194 & $-450,150$ & $1368 \pm 29$ & $949 \pm 21$ & $787 \pm 24$ & $650 \pm 42$ & $461 \pm 50$ & $\ldots$ \\
\hline $\mathrm{J} 1009+0713$ & 204_17 & 0.22784 & $-200,280$ & $982 \pm 27$ & $516 \pm 22$ & $\ldots$ & $\ldots$ & $\ldots$ & $\ldots$ \\
\hline $\mathrm{J} 1009+0713$ & 170_9 & 0.35569 & $-250,350$ & $1406 \pm 60$ & $1117 \pm 27$ & $978 \pm 26$ & $\ldots$ & $905 \pm 18$ & $\ldots$ \\
\hline $\mathrm{J} 1016+4706$ & 274_6 & 0.25195 & $-100,350$ & $1342 \pm 25$ & $1031 \pm 16$ & $\ldots$ & $\ldots$ & $735 \pm 23$ & $681 \pm 32$ \\
\hline $\mathrm{J} 1016+4706$ & 359_16 & 0.16614 & $-300,150$ & $959 \pm 22$ & $733 \pm 20$ & $\ldots$ & $\ldots$ & $\ldots$ & $\ldots$ \\
\hline J1112+3539 & 236_14 & 0.24670 & $-200,350$ & $1201 \pm 43$ & $624 \pm 22$ & $\ldots$ & $196 \pm 40$ & $232 \pm 67$ & $48 \pm 75$ \\
\hline $\mathrm{J} 1133+0327$ & $110 \_5$ & 0.23670 & $-50,600$ & $1663 \pm 37$ & $1103 \pm 20$ & $932 \pm 25$ & $913 \pm 30$ & $901 \pm 31$ & $826 \pm 61$ \\
\hline J1133+0327 & 164_21 & 0.15449 & $-300,100$ & $768 \pm 33$ & $424 \pm 23$ & $\ldots$ & $\ldots$ & $\ldots$ & $\ldots$ \\
\hline $\mathrm{J} 1157-0022$ & $230 \_7$ & 0.16378 & $-80,250$ & $587 \pm 22$ & $417 \pm 25$ & $\ldots$ & $\ldots$ & $\ldots$ & $\ldots$ \\
\hline $\mathrm{J} 1220+3853$ & $225 \_38$ & 0.27371 & $-400,200$ & $1173 \pm 46$ & $\ldots$ & $496 \pm 39$ & $399 \pm 56$ & $267 \pm 43$ & $123 \pm 33$ \\
\hline $\mathrm{J} 1233+4758$ & $94 \_38$ & 0.22210 & $-250,200$ & $1079 \pm 21$ & $768 \pm 17$ & $574 \pm 21$ & $485 \pm 23$ & $504 \pm 31$ & $323 \pm 41$ \\
\hline $\mathrm{J} 1233-0031$ & $168 \_7$ & 0.31850 & $-200,200$ & $910 \pm 30$ & $608 \pm 18$ & $416 \pm 22$ & $265 \pm 16$ & $176 \pm 17$ & $96 \pm 19$ \\
\hline $\mathrm{J} 1241+5721$ & 199_6 & 0.20526 & $-250,300$ & $1128 \pm 21$ & $732 \pm 16$ & $620 \pm 20$ & $538 \pm 41$ & $\ldots$ & $\ldots$ \\
\hline $\mathrm{J} 1241+5721$ & 208_27 & 0.21780 & $-550,250$ & $762 \pm 31$ & $457 \pm 13$ & $273 \pm 16$ & $136 \pm 25$ & $105 \pm 26$ & $\ldots$ \\
\hline $\mathrm{J} 1245+3356$ & 236_36 & 0.19248 & $-100,500$ & $823 \pm 30$ & $296 \pm 22$ & $101 \pm 25$ & $\ldots$ & $\ldots$ & $\ldots$ \\
\hline $\mathrm{J} 1322+4645$ & 349_11 & 0.21418 & $-150,300$ & $1059 \pm 26$ & $762 \pm 22$ & $683 \pm 26$ & $662 \pm 35$ & $546 \pm 39$ & $\ldots$ \\
\hline $\mathrm{J} 1330+2813$ & 289_28 & 0.19236 & $-250,300$ & $1255 \pm 22$ & $894 \pm 25$ & $769 \pm 26$ & $\ldots$ & $\ldots$ & $\ldots$ \\
\hline $\mathrm{J} 1342-0053$ & 157_10 & 0.22702 & $-300,500$ & $2149 \pm 26$ & $1321 \pm 11$ & $1039 \pm 16$ & $788 \pm 39$ & $\ldots$ & $\ldots$ \\
\hline $\mathrm{J} 1342-0053$ & 77_10 & 0.20127 & $-100,0$ & $\leqslant 14$ & $\leqslant 12$ & $\ldots$ & $\ldots$ & $\ldots$ & $\ldots$ \\
\hline J1419+4207 & 132_30 & 0.17925 & $-450,150$ & $1019 \pm 33$ & $\ldots$ & $351 \pm 54$ & $\ldots$ & $\ldots$ & $\ldots$ \\
\hline $\mathrm{J} 1435+3604$ & 126_21 & 0.26226 & $-450,200$ & $721 \pm 39$ & $\ldots$ & $232 \pm 32$ & $\ldots$ & $\ldots$ & $\ldots$ \\
\hline $\mathrm{J} 1435+3604$ & $68 \_12$ & 0.20237 & $-300,500$ & $4993 \pm 51$ & $1418 \pm 39$ & $827 \pm 65$ & $142 \pm 91$ & $\ldots$ & $\ldots$ \\
\hline $\mathrm{J} 1437+5045$ & 317_38 & 0.24600 & $-200,200$ & $626 \pm 57$ & $\leqslant 58$ & $\cdots$ & $\ldots$ & $\ldots$ & $\ldots$ \\
\hline $\mathrm{J} 1445+3428$ & 232_33 & 0.21764 & $-150,300$ & $676 \pm 39$ & $383 \pm 27$ & $193 \pm 22$ & $95 \pm 29$ & $\ldots$ & $\ldots$ \\
\hline J1514+3619 & 287_14 & 0.21223 & $-350,580$ & $1806 \pm 60$ & $\ldots$ & $\ldots$ & $\ldots$ & $\ldots$ & $\ldots$ \\
\hline $\mathrm{J} 1550+4001$ & 197_23 & 0.31247 & $-250,400$ & $1307 \pm 37$ & $660 \pm 27$ & $490 \pm 37$ & $441 \pm 18$ & $421 \pm 19$ & $344 \pm 23$ \\
\hline $\mathrm{J} 1550+4001$ & 97_33 & 0.32179 & $-150,170$ & $242 \pm 53$ & $\ldots$ & $\ldots$ & $\ldots$ & $\ldots$ & $\ldots$ \\
\hline $\mathrm{J} 1555+3628$ & 88_11 & 0.18930 & $-300,200$ & $926 \pm 27$ & $749 \pm 27$ & $547 \pm 36$ & $\cdots$ & $\ldots$ & $\ldots$ \\
\hline $\mathrm{J} 1617+0638$ & 253_39 & 0.15258 & $-50,50$ & $\leqslant 71$ & $\ldots$ & $\cdots$ & $\cdots$ & $\ldots$ & $\cdots$ \\
\hline $\mathrm{J} 1619+3342$ & $113 \_40$ & 0.14137 & $-200,150$ & $664 \pm 13$ & $335 \pm 16$ & $\cdots$ & $\ldots$ & $\cdots$ & $\cdots$ \\
\hline $\mathrm{J} 2257+1340$ & 270_40 & 0.17675 & 50,150 & $\leqslant 18$ & $\leqslant 21$ & $\ldots$ & $\ldots$ & $\ldots$ & $\ldots$ \\
\hline $\mathrm{J} 2345-0059$ & 356_12 & 0.25389 & $-200,150$ & $873 \pm 25$ & $559 \pm 23$ & $\cdots$ & $447 \pm 20$ & $345 \pm 23$ & $239 \pm 43$ \\
\hline
\end{tabular}

Notes.

a This velocity range is generally given for Ly $\alpha$, but replaced with the values for Ly $\beta$ where Ly $\alpha$ is damped, blended, or otherwise unavailable.

b Limits on $W_{r}$ are $1 \sigma$.

rare cases up to a full $\sim 20 \mathrm{~km} \mathrm{~s}^{-1}$ resolution element) owing to errors in the COS geometric distortion and/or wavelength solutions. To allow for these small instrumental shifts, our multi-line fitting allows for small velocity shifts relative to the strongest line (here, generally $\operatorname{Ly} \alpha$ ) as nuisance parameters whose fitted values are then ignored. Model intrinsic profiles are constructed with nuisance lines and velocity shifts applied. These intrinsic model profiles are then convolved with the COS line-spread function (LSF) as given at the nearest observedwavelength grid point in the compilation by Ghavamian et al. (2009).

The MPFIT software ${ }^{14}$ is used to do the optimization of the fit and to generate errors near the best fit point, which are

\footnotetext{
14 http://cow.physics.wisc.edu/ craigm/idl/fitting.html
}

stored in the corresponding line slice along with individual and total model profiles. These are formal errors, computed from parameter covariance matrices derived within MPFIT. These fits are subject to several sources of error affecting components at different column densities and $b$-values. For systems with strongly saturated Lyman series lines that do not yet exhibit damping wings $\left(\log N_{\mathrm{HI}} \sim 16.5-18.5\right)$, these formal errors likely underestimate the true uncertainty in the fitted column densities. In this column density range singlecomponent absorbers are on the flat part of the curve of growth, so that errors in $N_{\mathrm{H}_{\mathrm{I}}}$ and $b$ are correlated. Furthermore, we generally cannot discern individual component structure in these strongly saturated profiles (examples are J1016+4706 359_16 and J1322+4645 349_11), so only one component can be fitted, typically resulting in high $N_{\mathrm{H}_{\mathrm{I}}}$ and a single broad $b$ 
Table 5

The COS-Halos H I Sample

\begin{tabular}{|c|c|c|c|c|c|c|c|c|c|c|}
\hline \multirow[t]{2}{*}{ Sightline } & \multirow[t]{2}{*}{ Galaxy } & \multirow[t]{2}{*}{$z_{\text {sys }}$} & \multicolumn{4}{|c|}{ Adopted Values $^{\mathrm{b}}$} & \multicolumn{3}{|c|}{ Profile Fitting } & \multirow[t]{2}{*}{ Notes $^{c}$} \\
\hline & & & $\begin{array}{c}v_{\min }, v_{\max } \\
\left(\mathrm{km} \mathrm{s}^{-1}\right)\end{array}$ & Adopt & $\begin{array}{l}\log N_{\mathrm{HI}_{\mathrm{I}}} \\
\left(\mathrm{cm}^{-2}\right)\end{array}$ & Method & $\begin{array}{c}\log N_{\mathrm{HI}} \\
\left(\mathrm{cm}^{-2}\right)\end{array}$ & $\begin{array}{c}b \\
\left(\mathrm{~km} \mathrm{~s}^{-1}\right)\end{array}$ & $\begin{array}{c}v \\
\left(\mathrm{~km} \mathrm{~s}^{-1}\right)\end{array}$ & \\
\hline $\mathrm{J} 0226+0015$ & 268_22 & 0.22744 & 0,280 & 1 & $14.25 \pm 0.03$ & 1 & $\begin{array}{c}\ldots \\
13.03 \pm 0.16 \\
14.34 \pm 0.06\end{array}$ & $\begin{array}{c}\cdots \\
15 \pm 9 \\
40 \pm 3\end{array}$ & $\begin{array}{c}\cdots \\
67 \pm 5 \\
166 \pm 1\end{array}$ & Unc \\
\hline $\mathrm{J} 0401-0540$ & 67_24 & 0.21969 & $-700,120$ & 8 & $15.63 \pm 0.05$ & 1 & $\begin{array}{c}\cdots \\
13.63 \pm 0.07 \\
14.64 \pm 0.08 \\
15.54 \pm 0.09 \\
14.97 \pm 0.24\end{array}$ & $\begin{array}{c}\cdots \\
23 \pm 6 \\
19 \pm 1 \\
30 \pm 4 \\
55 \pm 5\end{array}$ & $\begin{array}{c}\ldots \\
-642 \pm 5 \\
-579 \pm 2 \\
-63 \pm 5 \\
-46 \pm 3\end{array}$ & \\
\hline $\mathrm{J} 0803+4332$ & 306_20 & 0.25347 & $-250,100$ & 1 & $14.78 \pm 0.04$ & 1 & $\begin{array}{c}\ldots \\
13.61 \pm 0.20 \\
14.68 \pm 0.14 \\
14.39 \pm 0.21\end{array}$ & $\begin{array}{c}\ldots \\
131 \pm 42 \\
17 \pm 5 \\
37 \pm 12\end{array}$ & $\begin{array}{c}\cdots \\
-78 \pm 48 \\
-50 \pm 6 \\
-3 \pm 18\end{array}$ & Broad \\
\hline J0910+1014 & $34 \_46$ & 0.14274 & $-300,300$ & 8 & $14.76 \pm 0.03$ & 1 & $\begin{array}{c}\ldots \\
15.74 \pm 0.53 \\
14.02 \pm 0.06\end{array}$ & $\begin{array}{c}\cdots \\
73 \pm 12 \\
48 \pm 7\end{array}$ & $\begin{array}{c}\cdots \\
-75 \pm 3 \\
171 \pm 5\end{array}$ & Sat \\
\hline J0910+1014 & 242_34 & 0.26412 & $-300,250$ & 8 & $15.34 \pm 0.09$ & 1 & $\begin{array}{c}\ldots \\
16.36 \pm 0.42 \\
14.40 \pm 0.11 \\
15.00 \pm 0.10 \\
14.31 \pm 0.19\end{array}$ & $\begin{array}{c}\cdots \\
15 \pm 1 \\
21 \pm 2 \\
30 \pm 4 \\
20 \pm 8\end{array}$ & $\begin{aligned} \cdots & \\
-154 & \pm 2 \\
-62 & \pm 2 \\
46 & \pm 6 \\
110 & \pm 10\end{aligned}$ & \\
\hline J0914+2823 & $41 \_27$ & 0.24431 & $-250,100$ & 1 & $15.45 \pm 0.03$ & 1 & $\begin{array}{c}\cdots \\
14.07 \pm 0.43 \\
15.57 \pm 0.03\end{array}$ & $\begin{array}{c}\cdots \\
61 \pm 28 \\
31 \pm 1\end{array}$ & $\begin{array}{c}\cdots \\
-106 \pm 47 \\
-40 \pm 3\end{array}$ & Unc \\
\hline J0925+4004 & 196_22 & 0.24745 & $-775,1025$ & 1 & $19.55 \pm 0.15$ & 3 & $\begin{array}{c}\cdots \\
19.62 \pm 0.02\end{array}$ & $\begin{array}{c}\cdots \\
35 \pm 1\end{array}$ & $\begin{array}{c}\cdots \\
65 \pm 5\end{array}$ & \\
\hline J0928+6025 & 110_35 & 0.15400 & $-700,700$ & 1 & $19.35 \pm 0.15$ & 3 & $\begin{array}{c}\ldots \\
19.44 \pm 0.03\end{array}$ & $\begin{array}{c}\cdots \\
35 \pm 1\end{array}$ & $\begin{array}{c}\cdots \\
-62 \pm 6\end{array}$ & \\
\hline J0935+0204 & 15_28 & 0.26228 & $-50,50$ & 4 & $<12.68$ & 1 & $\ldots$ & $\ldots$ & $\ldots$ & \\
\hline J0943+0531 & 106_34 & 0.22839 & 50,330 & 8 & $15.43 \pm 0.08$ & 1 & $\begin{array}{c}\cdots \\
15.45 \pm 0.08 \\
13.46 \pm 0.16\end{array}$ & $\begin{array}{c}\cdots \\
26 \pm 1 \\
12 \pm 6\end{array}$ & $\begin{array}{c}\cdots \\
185 \pm 2 \\
298 \pm 3\end{array}$ & Unc \\
\hline J0943+0531 & 216_61 & 0.14311 & $-180,50$ & 8 & $14.88 \pm 0.06$ & 1 & $\begin{array}{c}\ldots \\
15.21 \pm 0.32\end{array}$ & $\begin{array}{c}\cdots \\
30 \pm 8\end{array}$ & $\begin{array}{c}\cdots \\
-80 \pm 3\end{array}$ & Sat \\
\hline J0943+0531 & $227 \_19$ & 0.35295 & $-100,890$ & 1 & $16.29 \pm 0.03$ & 1 & $\begin{array}{c}\ldots \\
14.82 \pm 0.04 \\
14.14 \pm 0.69 \\
14.92 \pm 0.15 \\
16.12 \pm 0.04 \\
15.65 \pm 0.12 \\
15.46 \pm 0.19 \\
15.25 \pm 0.22 \\
13.53 \pm 0.26 \\
13.48 \pm 0.15\end{array}$ & $\begin{aligned} & \cdots \\
& 41 \pm 3 \\
& 46 \pm 45 \\
& 22 \pm 7 \\
& 27 \pm 2 \\
& 12 \pm 4 \\
& 14 \pm 8 \\
& 95 \pm 20 \\
& 7 \pm 5 \\
& 27 \pm 14\end{aligned}$ & $\begin{array}{c}\cdots \\
74 \pm 4 \\
233 \pm 62 \\
281 \pm 6 \\
363 \pm 6 \\
458 \pm 7 \\
426 \pm 9 \\
481 \pm 39 \\
719 \pm 3 \\
850 \pm 9\end{array}$ & $\begin{array}{l}\text { Broad } \\
\text { Unc } \\
\text { Unc }\end{array}$ \\
\hline J0950+4831 & 177_27 & 0.21194 & $-450,150$ & 8 & $16.18 \pm 0.06$ & 1 & $\begin{array}{c}\cdots \\
13.50 \pm 0.07 \\
16.88 \pm 0.13\end{array}$ & $\begin{array}{c}\cdots \\
38 \pm 8 \\
56 \pm 1\end{array}$ & $\begin{array}{c}\cdots \\
-322 \pm 5 \\
-96 \pm 2\end{array}$ & $\begin{array}{l}\text { Unc } \\
\text { Sat }\end{array}$ \\
\hline $\mathrm{J} 1009+0713$ & 204_17 & 0.22784 & $-200,280$ & 8 & $15.25 \pm 0.03$ & 1 & $\begin{array}{c}\ldots \\
14.90 \pm 0.28 \\
15.25 \pm 0.18 \\
13.47 \pm 0.12 \\
13.28 \pm 0.09\end{array}$ & $\begin{array}{c}\cdots \\
31 \pm 8 \\
37 \pm 9 \\
18 \pm 6 \\
16 \pm 6\end{array}$ & $\begin{aligned} & \ldots \\
&-53 \pm 16 \\
& 18 \pm 14 \\
& 119 \pm 4 \\
& 235 \pm 3\end{aligned}$ & $\begin{array}{c}\text { Sat } \\
\text { Sat } \\
\text { Ly } \alpha \text { only }\end{array}$ \\
\hline $\mathrm{J} 1009+0713$ & 170_9 & 0.35569 & $-250,350$ & 8 & $18.00 \pm-9.99$ & 4 & $\begin{array}{c}\ldots \\
16.24 \pm 0.08 \\
18.40 \pm 0.22 \\
15.17 \pm 0.07\end{array}$ & $\begin{array}{c}\cdots \\
34 \pm 3 \\
33 \pm 1 \\
18 \pm 1\end{array}$ & $\begin{array}{r}\ldots \\
-67 \pm 7 \\
47 \pm 5 \\
206 \pm 3\end{array}$ & $\begin{array}{l}\text { Sat } \\
\text { Sat } \\
\text { Unc }\end{array}$ \\
\hline J1016+4706 & 274_6 & 0.25195 & $-100,350$ & 8 & $16.59 \pm 0.04$ & 1 & $\begin{array}{c}\cdots \\
17.89 \pm 0.20 \\
16.14 \pm 0.04\end{array}$ & $\begin{array}{c}\cdots \\
25 \pm 1 \\
54 \pm 3\end{array}$ & $\begin{array}{c}\cdots \\
34 \pm 4 \\
154 \pm 6\end{array}$ & $\begin{array}{l}\text { Sat } \\
\text { Sat }\end{array}$ \\
\hline $\mathrm{J} 1016+4706$ & $359 \_16$ & 0.16614 & $-300,150$ & 8 & $15.44 \pm 0.03$ & 1 & $\begin{array}{c}\ldots \\
17.34 \pm 0.44\end{array}$ & $\begin{array}{c}\cdots \\
42 \pm 3\end{array}$ & $\begin{array}{c}\cdots \\
-81 \pm 1\end{array}$ & Sat \\
\hline $\mathrm{J} 1112+3539$ & 236_14 & 0.24670 & $-200,350$ & 8 & $15.79 \pm 0.13$ & 1 & $\begin{array}{c}\ldots \\
15.64 \pm 0.16 \\
15.22 \pm 0.11\end{array}$ & $\begin{array}{c}\cdots \\
19 \pm 1 \\
20 \pm 2\end{array}$ & $\begin{array}{r}\ldots \\
-81 \pm 3 \\
6 \pm 4\end{array}$ & \\
\hline
\end{tabular}


Table 5

(Continued)

\begin{tabular}{|c|c|c|c|c|c|c|c|c|c|c|}
\hline \multirow[t]{2}{*}{ Sightline } & \multirow[t]{2}{*}{ Galaxy } & \multirow[t]{2}{*}{$z_{\text {sys }}$} & \multicolumn{4}{|c|}{ Adopted Values ${ }^{\mathrm{b}}$} & \multicolumn{3}{|c|}{ Profile Fitting } & \multirow[t]{2}{*}{ Notes $^{c}$} \\
\hline & & & $\begin{array}{c}v_{\min }, v_{\max } \\
\left(\mathrm{km} \mathrm{s}^{-1}\right)\end{array}$ & Adopt & $\begin{array}{l}\log N_{\mathrm{HI}} \\
\left(\mathrm{cm}^{-2}\right)\end{array}$ & Method & $\begin{array}{l}\log N_{\mathrm{HI}} \\
\left(\mathrm{cm}^{-2}\right)\end{array}$ & $\begin{array}{c}b \\
\left(\mathrm{~km} \mathrm{~s}^{-1}\right)\end{array}$ & $\begin{array}{c}v \\
\left(\mathrm{~km} \mathrm{~s}^{-1}\right)\end{array}$ & \\
\hline & & & & & & & $\begin{array}{l}14.44 \pm 0.08 \\
13.47 \pm 0.11\end{array}$ & $\begin{array}{l}55 \pm 11 \\
49 \pm 17\end{array}$ & $\begin{array}{r}87 \pm 12 \\
269 \pm 11\end{array}$ & Unc \\
\hline $\mathrm{J} 1133+0327$ & $110 \_5$ & 0.23670 & $-50,600$ & 1 & $18.61 \pm 0.06$ & 3 & $\begin{array}{c}\ldots \\
18.65 \pm 0.06 \\
16.10 \pm 0.12 \\
17.57 \pm 0.25\end{array}$ & $\begin{array}{c}\ldots \\
24 \pm 1 \\
20 \pm 2 \\
18 \pm 1\end{array}$ & $\begin{array}{c}\ldots \\
168 \pm 3 \\
267 \pm 4 \\
390 \pm 2\end{array}$ & $\begin{array}{l}\text { Sat } \\
\text { Sat }\end{array}$ \\
\hline $\mathrm{J} 1133+0327$ & $164 \_21$ & 0.15449 & $-300,100$ & 8 & $15.09 \pm 0.04$ & 1 & $\begin{array}{c}\ldots \\
13.45 \pm 0.35 \\
15.58 \pm 0.21\end{array}$ & $\begin{array}{c}\cdots \\
27 \pm 15 \\
34 \pm 2\end{array}$ & $\begin{array}{c}\ldots \\
-213 \pm 5 \\
-76 \pm 2\end{array}$ & $\begin{array}{l}\text { Unc } \\
\text { Sat }\end{array}$ \\
\hline $\mathrm{J} 1157-0022$ & $230 \_7$ & 0.16378 & $-80,250$ & 8 & $15.12 \pm 0.04$ & 1 & $\begin{array}{c}\ldots \\
14.46 \pm 0.66 \\
15.67 \pm 0.58\end{array}$ & $\begin{array}{c}\ldots \\
7 \pm 7 \\
26 \pm 8\end{array}$ & $\begin{aligned} & \cdots \\
& 13 \pm 9 \\
& 75 \pm 10\end{aligned}$ & $\begin{array}{l}\text { Unc } \\
\text { Sat }\end{array}$ \\
\hline $\mathrm{J} 1220+3853$ & $225 \_38$ & 0.27371 & $-400,200$ & 1 & $15.83 \pm 0.05$ & 1 & $\begin{array}{c}\ldots \\
14.78 \pm 0.08 \\
15.83 \pm 0.06 \\
14.73 \pm 0.10 \\
14.56 \pm 0.30\end{array}$ & $\begin{aligned} & \ldots \\
44 & \pm 8 \\
23 & \pm 1 \\
13 & \pm 1 \\
4 & \pm 1\end{aligned}$ & $\begin{aligned} \cdots & \\
6 & \pm 10 \\
-81 & \pm 2 \\
-336 & \pm 2 \\
117 & \pm 2\end{aligned}$ & Unc \\
\hline $\mathrm{J} 1233+4758$ & 94_38 & 0.22210 & $-250,200$ & 8 & $16.28 \pm 0.06$ & 1 & $\begin{array}{c}\ldots \\
16.34 \pm 0.28 \\
18.09 \pm 0.29 \\
15.51 \pm 0.11 \\
14.08 \pm 0.07\end{array}$ & $\begin{aligned} & \ldots \\
& 35 \pm 4 \\
& 9 \pm 6 \\
& 15 \pm 3 \\
& 12 \pm 2\end{aligned}$ & $\begin{aligned} \ldots & \\
0 & \pm 13 \\
-38 & \pm 17 \\
-91 & \pm 6 \\
-149 & \pm 2\end{aligned}$ & $\begin{array}{l}\text { Sat } \\
\text { Sat }\end{array}$ \\
\hline $\mathrm{J} 1233-0031$ & $168 \_7$ & 0.31850 & $-200,200$ & 1 & $15.57 \pm 0.02$ & 1 & $\begin{array}{c}\cdots \\
15.03 \pm 0.19 \\
15.46 \pm 0.08 \\
14.33 \pm 0.06\end{array}$ & $\begin{array}{c}\ldots \\
28 \pm 5 \\
30 \pm 4 \\
29 \pm 4\end{array}$ & $\begin{aligned} & \cdots \\
86 & \pm 12 \\
34 & \pm 7 \\
-56 & \pm 5\end{aligned}$ & \\
\hline $\mathrm{J} 1241+5721$ & $199 \_6$ & 0.20526 & $-250,300$ & 8 & $16.06 \pm 0.04$ & 1 & $\begin{array}{c}\ldots \\
14.03 \pm 0.05 \\
17.96 \pm 0.14\end{array}$ & $\begin{array}{c}\ldots \\
81 \pm 9 \\
34 \pm 1\end{array}$ & $\begin{array}{c}\ldots \\
-30 \pm 1 \\
61 \pm 1\end{array}$ & $\begin{array}{l}\text { Unc } \\
\text { Sat }\end{array}$ \\
\hline $\mathrm{J} 1241+5721$ & 208_27 & 0.21780 & $-550,250$ & 1 & $15.30 \pm 0.06$ & 1 & $\begin{array}{c}\ldots \\
13.40 \pm 0.06 \\
13.01 \pm 0.70 \\
15.34 \pm 0.08 \\
14.55 \pm 0.25\end{array}$ & $\begin{array}{c}\ldots \\
38 \pm 7 \\
1 \pm 2 \\
20 \pm 2 \\
41 \pm 10\end{array}$ & $\begin{aligned} & \ldots \\
&-424 \pm 4 \\
&-34 \pm 1 \\
& 22 \pm 3 \\
& 67 \pm 18\end{aligned}$ & $\begin{array}{l}\text { Ly } \alpha \text { only } \\
\text { Unc }\end{array}$ \\
\hline $\mathrm{J} 1245+3356$ & 236_36 & 0.19248 & $-100,500$ & 1 & $14.76 \pm 0.04$ & 1 & $\begin{array}{c}\cdots \\
14.68 \pm 0.04 \\
13.84 \pm 0.04\end{array}$ & $\begin{array}{c}\cdots \\
36 \pm 1 \\
63 \pm 7\end{array}$ & $\begin{array}{c}\ldots \\
11 \pm 1 \\
370 \pm 4\end{array}$ & Ly $\alpha$ only \\
\hline $\mathrm{J} 1322+4645$ & 349_11 & 0.21418 & $-150,300$ & 8 & $16.33 \pm 0.04$ & 1 & $\begin{array}{c}\cdots \\
18.02 \pm 0.15\end{array}$ & $\begin{array}{c}\ldots \\
40 \pm 1\end{array}$ & $\begin{array}{c}\ldots \\
71 \pm 1\end{array}$ & Sat \\
\hline $\mathrm{J} 1330+2813$ & 289_28 & 0.19236 & $-250,300$ & 8 & $15.88 \pm 0.03$ & 1 & $\begin{array}{c}\cdots \\
17.64 \pm 0.24 \\
18.26 \pm 0.06\end{array}$ & $\begin{array}{c}\ldots \\
24 \pm 1 \\
17 \pm 1\end{array}$ & $\begin{array}{r}\cdots \\
128 \pm 2 \\
1 \pm 2\end{array}$ & $\begin{array}{l}\text { Sat } \\
\text { Sat }\end{array}$ \\
\hline $\mathrm{J} 1342-0053$ & 157_10 & 0.22702 & $-300,500$ & 8 & $16.27 \pm 0.03$ & 1 & $\begin{array}{c}\ldots \\
14.80 \pm 0.27 \\
18.92 \pm 0.04 \\
13.94 \pm 0.08 \\
14.55 \pm 0.07\end{array}$ & $\begin{aligned} & \ldots \\
6 & \pm 1 \\
47 & \pm 1 \\
18 & \pm 2 \\
15 & \pm 1\end{aligned}$ & $\begin{aligned} & \ldots \\
&-95 \pm 1 \\
& 72 \pm 2 \\
& 304 \pm 1 \\
& 419 \pm 1\end{aligned}$ & $\begin{array}{l}\text { Unc } \\
\text { Sat }\end{array}$ \\
\hline $\mathrm{J} 1342-0053$ & 77_10 & 0.20127 & $-100,0$ & 4 & $<12.43$ & 1 & $\ldots$ & $\ldots$ & $\ldots$ & \\
\hline $\mathrm{J} 1419+4207$ & 132_30 & 0.17925 & $-450,150$ & 8 & $15.42 \pm 0.07$ & 1 & $\begin{array}{c}\ldots \\
13.12 \pm 0.16 \\
12.85 \pm 0.29 \\
18.26 \pm 0.05 \\
14.35 \pm 1.34 \\
15.66 \pm 3.51\end{array}$ & $\begin{aligned} & \cdots \\
& 12 \pm 10 \\
& 17 \pm 22 \\
& 14 \pm 2 \\
& 22 \pm 48 \\
& 5 \pm 10\end{aligned}$ & $\begin{aligned} & \ldots \\
&-389 \pm 6 \\
&-343 \pm 12 \\
&-95 \pm 6 \\
&-32 \pm 73 \\
& 10 \pm 23\end{aligned}$ & $\begin{array}{l}\text { Unc } \\
\text { Unc } \\
\text { Sat } \\
\text { Unc } \\
\text { Unc }\end{array}$ \\
\hline $\mathrm{J} 1435+3604$ & $126 \_21$ & 0.26226 & $-450,200$ & 1 & $15.25 \pm 0.06$ & 1 & $\begin{array}{c}\ldots \\
13.30 \pm 0.09 \\
15.02 \pm 0.09 \\
15.09 \pm 0.18\end{array}$ & $\begin{array}{c}\ldots \\
21 \pm 7 \\
46 \pm 2 \\
12 \pm 4\end{array}$ & $\begin{aligned} & \ldots \\
&-381 \pm 4 \\
& 42 \pm 2 \\
& 78 \pm 8\end{aligned}$ & Ly $\alpha$ only \\
\hline $\mathrm{J} 1435+3604$ & 68_12 & 0.20237 & $-1500,1500$ & 1 & $19.80 \pm 0.10$ & 3 & $\begin{array}{c}\ldots \\
14.71 \pm 0.05 \\
19.95 \pm 0.01\end{array}$ & $\begin{array}{c}\cdots \\
47 \pm 6 \\
32 \pm 1\end{array}$ & $\begin{array}{c}\ldots \\
318 \pm 5 \\
99 \pm 2\end{array}$ & \\
\hline $\mathrm{J} 1437+5045$ & 317_38 & 0.24600 & $-200,200$ & 1 & $14.53 \pm 0.12$ & 3 & $\begin{array}{c}\cdots \\
13.63 \pm 0.70 \\
13.65 \pm 0.36\end{array}$ & $\begin{array}{r}\ldots \\
5 \pm 4 \\
44 \pm 1\end{array}$ & $\begin{array}{c}\ldots \\
-151 \pm 3 \\
-60 \pm 27\end{array}$ & Unc \\
\hline
\end{tabular}


Table 5

(Continued)

\begin{tabular}{|c|c|c|c|c|c|c|c|c|c|c|}
\hline \multirow[t]{2}{*}{ Sightline } & \multirow[t]{2}{*}{ Galaxy } & \multirow[t]{2}{*}{$z_{\text {sys }}$} & \multicolumn{4}{|c|}{ Adopted Values ${ }^{\text {b }}$} & \multicolumn{3}{|c|}{ Profile Fitting } & \multirow[t]{2}{*}{ Notes $^{\mathrm{c}}$} \\
\hline & & & $\begin{array}{c}v_{\min }, v_{\max } \\
\left(\mathrm{km} \mathrm{s}^{-1}\right)\end{array}$ & Adopt & $\begin{array}{l}\log N_{\mathrm{HI}} \\
\left(\mathrm{cm}^{-2}\right)\end{array}$ & Method & $\begin{array}{l}\log N_{\mathrm{HI}_{\mathrm{I}}} \\
\left(\mathrm{cm}^{-2}\right)\end{array}$ & $\begin{array}{c}b \\
\left(\mathrm{~km} \mathrm{~s}^{-1}\right)\end{array}$ & $\begin{array}{c}v \\
\left(\mathrm{~km} \mathrm{~s}^{-1}\right)\end{array}$ & \\
\hline & & & & & & & $\begin{array}{l}14.52 \pm 0.14 \\
13.69 \pm 0.15\end{array}$ & $\begin{array}{l}25 \pm 5 \\
17 \pm 6\end{array}$ & $\begin{array}{r}-5 \pm 7 \\
106 \pm 4\end{array}$ & \\
\hline $\mathrm{J} 1445+3428$ & 232_33 & 0.21764 & $-150,300$ & 1 & $15.07 \pm 0.06$ & 1 & $\begin{array}{c}\ldots \\
15.02 \pm 0.05 \\
13.81 \pm 0.16\end{array}$ & $\begin{array}{c}\cdots \\
32 \pm 2 \\
49 \pm 17\end{array}$ & $\begin{aligned} \cdots & \\
99 & \pm 4 \\
1 & \pm 16\end{aligned}$ & \\
\hline $\mathrm{J} 1514+3619$ & 287_14 & 0.21223 & $-350,580$ & 8 & $14.83 \pm 0.02$ & 1 & $\begin{array}{c}\ldots \\
18.14 \pm 0.25 \\
13.51 \pm 0.17 \\
13.82 \pm 0.07 \\
14.03 \pm 0.05\end{array}$ & $\begin{aligned} & \cdots \\
40 & \pm 2 \\
50 & \pm 24 \\
46 & \pm 8 \\
51 & \pm 6\end{aligned}$ & $\begin{aligned} \ldots & \\
-115 & \pm 3 \\
90 & \pm 14 \\
258 & \pm 6 \\
489 & \pm 5\end{aligned}$ & $\begin{array}{c}\text { Sat } \\
\text { Unc } \\
\text { Ly } \alpha \text { only } \\
\text { Ly } \alpha \text { only }\end{array}$ \\
\hline $\mathrm{J} 1550+4001$ & 197_23 & 0.31247 & $-250,400$ & 1 & $16.50 \pm 0.03$ & 1 & $\begin{array}{c}\cdots \\
14.54 \pm 0.07 \\
16.26 \pm 0.07 \\
16.32 \pm 0.04\end{array}$ & $\begin{array}{c}\ldots \\
64 \pm 9 \\
13 \pm 2 \\
36 \pm 2\end{array}$ & $\begin{aligned} \ldots & \\
134 & \pm 11 \\
54 & \pm 5 \\
1 & \pm 6\end{aligned}$ & \\
\hline $\mathrm{J} 1550+4001$ & 97_33 & 0.32179 & $-150,170$ & 1 & $13.86 \pm 0.09$ & 1 & $\begin{array}{c}\ldots \\
13.94 \pm 0.07\end{array}$ & $\begin{array}{c}\ldots \\
86 \pm 16\end{array}$ & $\begin{array}{l}\ldots \\
10 \pm 11\end{array}$ & Broad \\
\hline $\mathrm{J} 1555+3628$ & 88_11 & 0.18930 & $-300,200$ & 8 & $15.73 \pm 0.04$ & 1 & $\begin{array}{c}\ldots \\
18.17 \pm 0.08\end{array}$ & $\begin{array}{c}\ldots \\
31 \pm 1\end{array}$ & $\begin{array}{c}\ldots \\
-67 \pm 1\end{array}$ & Sat \\
\hline $\mathrm{J} 1617+0638$ & 253_39 & 0.15258 & $-50,50$ & 4 & $<13.12$ & 1 & $\ldots$ & $\ldots$ & $\ldots$ & \\
\hline $\mathrm{J} 1619+3342$ & $113 \_40$ & 0.14137 & $-200,150$ & 8 & $14.96 \pm 0.03$ & 1 & $\begin{array}{c}\ldots \\
15.06 \pm 0.12 \\
14.27 \pm 0.12\end{array}$ & $\begin{array}{c}\ldots \\
24 \pm 3 \\
38 \pm 5\end{array}$ & $\begin{array}{r}\cdots \\
6 \pm 4 \\
-55 \pm 8\end{array}$ & Sat \\
\hline $\mathrm{J} 2257+1340$ & 270_40 & 0.17675 & 50,150 & 4 & $<12.53$ & 1 & $\ldots$ & $\ldots$ & $\ldots$ & \\
\hline $\mathrm{J} 2345-0059$ & 356_12 & 0.25389 & $-200,150$ & 1 & $16.00 \pm 0.04$ & 1 & $\begin{array}{c}\cdots \\
13.72 \pm 0.39 \\
15.72 \pm 0.07 \\
15.77 \pm 0.09\end{array}$ & $\begin{array}{c}\cdots \\
22 \pm 14 \\
33 \pm 6 \\
23 \pm 4\end{array}$ & $\begin{aligned} \cdots & \\
97 & \pm 19 \\
16 & \pm 11 \\
-51 & \pm 9\end{aligned}$ & Unc \\
\hline
\end{tabular}

Notes.

${ }^{\text {a }}$ Columns are: QSO sightline, galaxy name, systemic redshift, minimum and maximum limits of direct integration, flag indicating a column density measurement (1, measurement; 4 , upper limit; 8 , lower limit), adopted column density and error, flag indicating method for column density estimate ( 1 , direct integration; 3 , fitted damping wings; 4, Lyman limit), and three parameters for the profile fits.

${ }^{b}$ Adopted column densities in this column are derived from direct integrations (Method =1) over the apparent optical depth profile (Savage \& Sembach 1991), except the damped systems for which profile-fitted values are always used (Method = 3) and the LLS toward J1009+0713 where the column density was derived from the Lyman limit (Method $=4$ ).

c Components labeled "Unc" have uncertain fits with large errors on parameters, or a doubtful identification as real absorption. Those labeled "Broad" have fits demanding an unusually broad linewidth, which may be spurious. "Sat" marks strongly saturated components with uncertain column densities. Fits derived from Ly $\alpha$ only are so indicated.

value. Thus, in the analysis that follows we typically use the more conservative lower limits to $N_{\mathrm{HI}}$ derived from apparent optical depth measurements of the highest available Lyman line. Assignment of a single component most likely overestimates the true $b$-value in these cases, so the formally derived $b$ can be considered reasonable upper limits for the characterization of gas kinematics. It can also be difficult to recognize a broad and shallow component (as would be expected for $\mathrm{H}$ i in hot gas at $>10^{5} \mathrm{~K}$ ) when it is superimposed on profiles with complex and strong narrow components. Moreover, even when evidence of broad components is present, its interpretation is often ambiguous (e.g., Figures 33-35 in Tripp et al. 2008). In some systems broad features arising in continuum fluctuations or noisy, blended weak components can appear as a broad single profile giving very large $b$-values in the formal fits. Component fits that are considered uncertain because of saturation, poor data quality, or unreliable parameters are flagged as such in Table 5 and identified in figures.

\subsection{Errors, Biases and Problems}

The COS-Halos database includes information from many sources: SDSS photometry, Keck and Magellan spectroscopy, and COS spectroscopy. To assess the robustness and statistical significance of our results, it is important to budget for both statistical and systematic errors in the measured and derived quantities. Our error budgets for galaxies and absorbers are displayed in Table 3.

The saturation of the Lyman series lines, even the intrinsically weak higher series lines, is a significant problem affecting a large portion of our systems. The saturation effect means that the lower limit we adopt for $N_{\mathrm{HI}}$ depends predominantly on the highest available Lyman line, which depends in turn on the system redshift (given a fixed short-wavelength cutoff in the COS detector). This strong saturation versus redshift effect is seen in Figure 6, where the points with lower limits follow a clear trend with redshift. As redshift increases, higher Lyman series lines become available and the minimum reliable column density estimate increases as well. The dashed line in the figure roughly indicates the region where column densities are high enough to be saturated in the highest available line. Note that because saturation flags are set based on local factors such as $\mathrm{S} / \mathrm{N}$ and line-profile shape, there is no one-to-one correspondence between saturation and column density and this line is just an approximation. Symbols without arrows 


$$
\begin{aligned}
& \mathrm{J} 0914+2823 \mathrm{z}_{\mathrm{QSO}}=0.7350 \quad-\text { Good } \quad \text { Non-detection } \\
& \text { 41_27 } \mathrm{z}_{\mathrm{sys}}=0.244314 \quad-\mathrm{Bad} \quad \text { - Saturated } \\
& \mathrm{SFR}=2.83 \text { hbeta } \\
& \text { - Blend } \\
& \mathrm{u}-\mathrm{r}=1.24 \quad \mathrm{R}=104.42 \\
& \log \mathrm{M}^{*}=9.63
\end{aligned}
$$

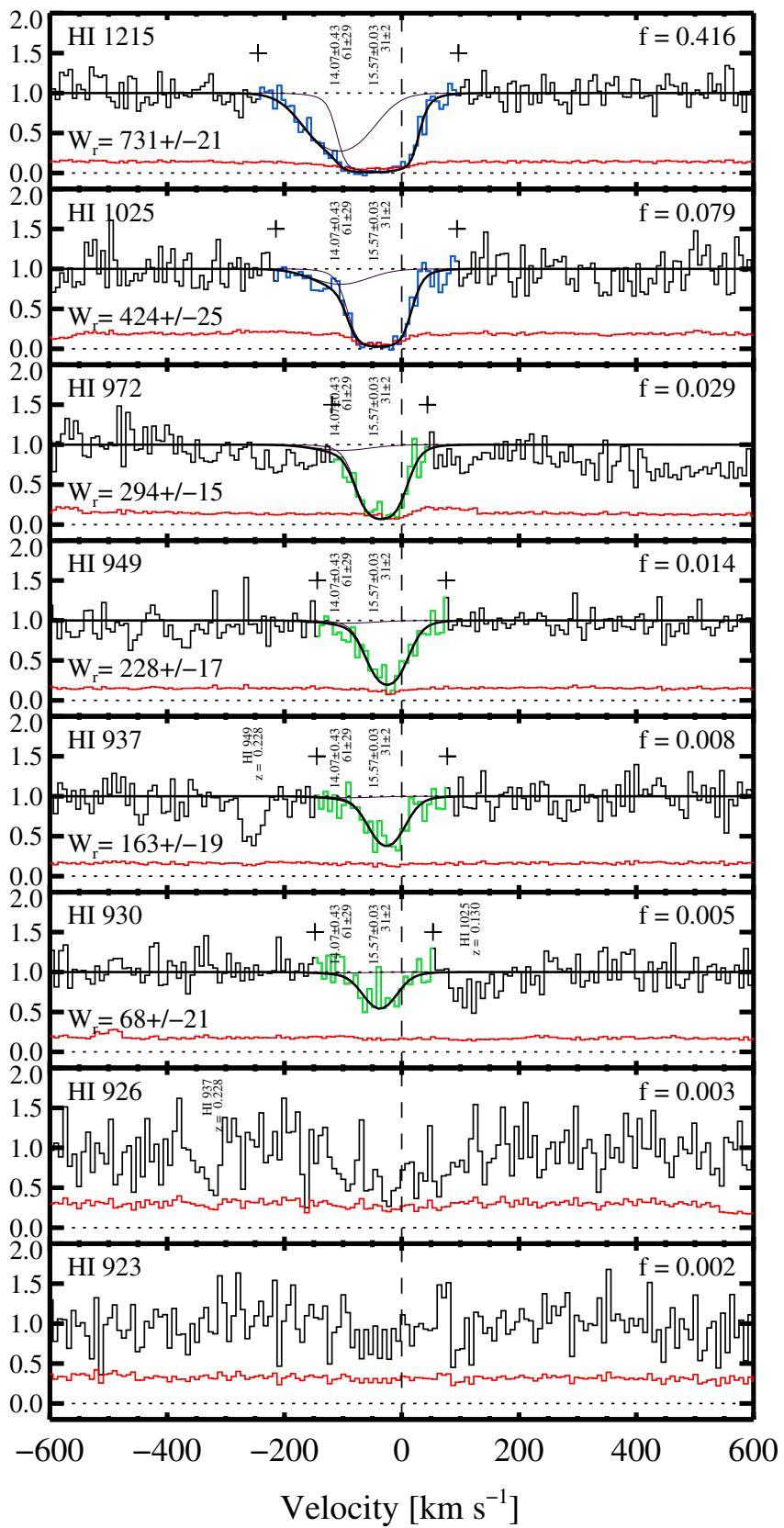

Figure 5. Stack plot illustrating our data and its analysis. These panels show $\mathrm{H}$ I absorption in the rest frame of the targeted galaxy 41_27 toward J0914+2823. All data is shifted to place the rest-frame of the galaxy systemic velocity at $v=0$. Galaxy quantities are shown at the top. Line quality flags are listed at the top and color-coded in the individual panels, where $W_{r}$ measurements are also displayed. Lines identified with other absorption-line systems are marked with their species and redshift in rotated text. The profile fits to individual components are shown in thin purple lines and their fitted column density $N_{\mathrm{H}}$ and Doppler $b$ are given above positioned at the component's central velocity. The composite profile fit is shown in the heavy black line. Profile fits have been convolved with the nominal COS LSFs for comparison to the data.

(A color version and the complete figure set (48 images) are available in the online journal.)

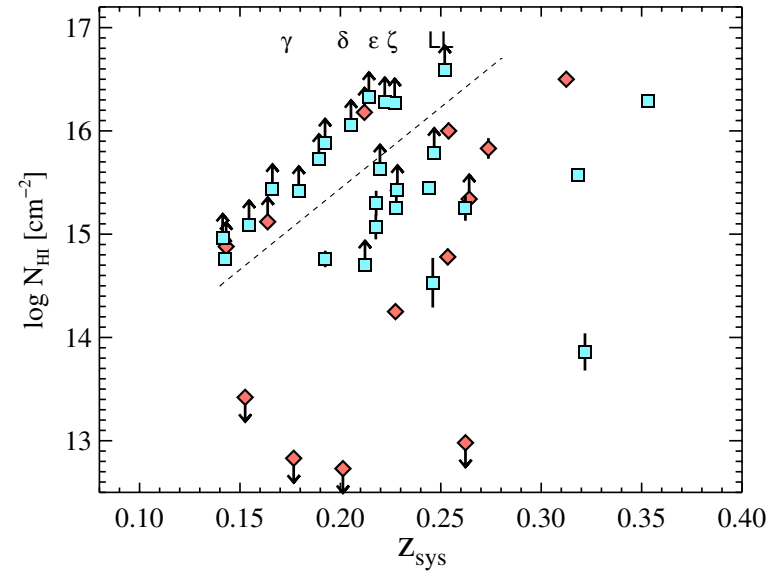

Figure 6. Illustration of the effects of saturation in the COS-Halos sample using adopted $N_{\mathrm{H}}$ column densities vs. redshift. For systems with upward pointing arrows we can infer only a lower limit to the column density of $\mathrm{H}$ I because of saturation. The Hi limit depends mainly on the redshift, through the strength of the highest available Lyman series line. The dashed line roughly indicates the boundary of the saturation region. Thus the $\mathrm{H}$ I limit increases with redshift as weaker lines appear on the G130M detector at $\lambda \gtrsim 1140 \AA$. The redshift at which each line appears on the COS detector is marked by the corresponding Greek letter. The lower limits below the indicative saturation line are screened by higher-redshift Lyman-limit systems, so that the weakest line they have available is not determined by their redshift.

(A color version of this figure is available in the online journal.)

are systems for which the $\mathrm{H}$ I is weak enough to give a clean measurement. ${ }^{15}$

Component structure, both resolved and unresolved, is an important issue affecting the analysis and interpretation of our results. Multiple components of absorption are often seen in the weaker Lyman series profiles. In many of these cases the components are fully blended, at least at COS resolution, and so inseparable in the Ly $\alpha$ profile. In many cases components are seen in Ly $\alpha$ that are too weak to be detected in the higher transitions. The simultaneous fitting of multiple Lyman series lines accounts for this effect by using component information in the higher lines and then self-consistently modeling the stronger transitions. But even this improvement imperfectly captures the true underlying component structure, which usually can only be seen in the weaker lines. Components that contribute to Ly $\alpha$ but not, e.g., $\operatorname{Ly} \beta$, will not always be included in the model. In most of our tests below we consider the total $N_{\mathrm{HI}}$ measured from either the total $W_{r}$ or by fitted components, but it must be recalled that these are only approximations to the true $N_{\mathrm{HI}}$ and its distribution into components. In our interpretations below we use metrics that are robust to these considerations, where possible.

\subsection{Optical Data Analysis}

Comparing the properties of CGM gas to the properties of the host galaxies requires robust redshifts, masses, star formation rates (SFRs), colors, and metallicities for the targeted galaxies. To measure these quantities we obtained medium-resolution optical spectroscopy using the LRIS spectrograph at Keck and the MagE spectrograph at Magellan. The details of data collection, reduction, and analysis for the COS-Halos optical

\footnotetext{
15 Four systems outside the saturation region possess lower limits because their highest Lyman series lines are screened by foreground Lyman-limit systems or other absorption at a different redshift, so the highest Lyman line for which we can obtain a measurement is not accurately reflected by their redshift alone.
} 
data are presented in Werk et al. (2012). Here we describe only those aspects of the measurements that are necessary to evaluate the CGM properties of the galaxies. The quantities derived from optical spectroscopy are listed in Table 2 and their errors in Table 3.

The high quality of the SDSS photometry and the groundbased spectroscopy mean that the most significant errors in Table 3 affecting galaxy quantities are not statistical errors from noise in the data. Rather, the most important galaxy uncertainties are systematic: the calibration of the stellar mass derivations, including the initial mass function (IMF) (10), the SFR (11), and the calibration of the metallicity scale (13). These errors are consistent with the literature on studies of similar type.

The galaxy spectroscopic redshifts $z_{\text {sys }}$ and the systemic velocity scales below were derived from a cross-correlation of the available nebular emission and/or stellar absorption lines. The $25 \mathrm{~km} \mathrm{~s}^{-1}$ error in the galaxy systemic velocity is a predominantly systematic error caused by instrumental effects such as flexure. The galaxy colors (e.g., $u-r$ ) and stellar masses $M_{*}$ were derived from the five-band SDSS photometry using the template-fitting approach implemented in the kcorrect code (Blanton \& Roweis 2007) and the measured $z_{\text {sys }}$. Errors in color are from the underlying SDSS photometry. Systematic errors from the mass-to-light ratio and IMF dominate the $\pm 0.2 \mathrm{dex}$ error in $M_{*}$. SFRs are estimated from the detected nebular emission lines or limited by their absence, with errors up to $\pm 50 \%$. For passive galaxies the SFR is given as a $2 \sigma$ upper limit. Errors on combined quantities, such as the specific star formation rate $\mathrm{sSFR}=\mathrm{SFR} / M_{*}$, are obtained from quadrature sums of the basic terms as specified in Table 3.

We compute halo masses $M_{\text {halo }}$ by interpolating along the abundance-matching relation of Moster et al. (2010) at the stellar mass determined by kcorrect from the SDSS ugriz photometry of the galaxy. We then determine the virial radius, $R_{\mathrm{vir}}$, with the relation

$$
R_{\text {vir }}=\left(3 M_{\text {halo }} / \Delta_{\text {vir }} \rho_{\text {crit }} 4 \pi\right)^{1 / 3},
$$

where $\rho_{\text {crit }}$ is the critical density at the spectroscopically determined redshift of the galaxy and $\Delta_{\mathrm{vir}}=200$. Accounting for systematic errors in the $M_{*}$ estimates (Table 3 ) and the scatter and uncertainty in the $M_{\text {halo }}-M_{*}$ relation, we adopt uncertainty of $50 \%$ on $R_{\text {vir }}$.

We have also observed most of these target QSOs with Keck HIRES to measure the near-UV and optical-band absorption from neutrals and low ions such as Fe II and $\mathrm{Mg}$ II. These data are described in Werk et al. (2013).

\section{THE H I CGM AS CHARACTERIZED BY COS-HALOS}

The COS-Halos data measure the incidence, strength, and kinematics of $\mathrm{H}$ I and metal-line absorption surrounding galaxies as a function of galaxy stellar mass and out to impact parameter $\simeq 150 \mathrm{kpc}$. We have already published results from the COS-Halos survey of O VI absorption surrounding galaxies, and concluded that $\mathrm{O}$ VI traces a significant reservoir of metals in a highly ionized CGM (Tumlinson et al. 2011a). An analysis of the COS-Halos sample of lower metallic ions (e.g., C II/III/IV, Si II/III/IV) has been published separately by Werk et al. (2013). The remainder of this paper is focused on the COS-Halos survey of $\mathrm{H}$ I absorption in the sample.

This section presents the key COS-Halos results on $\mathrm{HI}$ surrounding galaxies. In Section 4.1, we examine the strength of the $\mathrm{H}$ I absorption with equivalent widths and column densities. Section 4.2 examines the kinematics of the detected $\mathrm{H}$ I with respect to the galaxies. Section 4.3 examines the internal kinematics and linewidths of the detected gas. We compare the star-forming and passive galaxy subsamples to one another in all these sections (and later in Section 5.3). These results are compared to other studies in Section 5 and to interpretive models in Section 6. We summarize our chief results and remark on open questions in Section 7.

\subsection{Strong H I: Equivalent Widths and Column Densities}

The COS-Halos survey allows us to assess the quantity of $\mathrm{H} \mathrm{I}$ near galaxies, and to compare these quantities and trends with galaxy type, mass, and impact parameter. We perform these comparisons in terms of either $\operatorname{Ly} \alpha$ equivalent width (in the rest frame of the absorber, $W_{r}$ ), or in terms of column density $N_{\mathrm{H} \text { I }}$ (for complete systems, or by component). Both quantities carry useful information, but offer different advantages and suffer different shortcomings. Considering Ly $\alpha W_{r}$ only restricts the comparisons to well-defined measurements with no major systematic error and a small degree of censoring owing to nondetections, at the cost of losing the more physical information contained in $N_{\mathrm{H}}$. Conversely, considering column densities allows for the calculation of important physical quantities such as the absorber mass and size, but greatly increases the degree of censoring owing to saturation, which converts many wellmeasured equivalent widths into lower limits on $N_{\mathrm{HI}}$. In the analysis that follows, we endeavor to use the quantity best suited for the test in question, but these limitations must be borne in mind.

The complete sample of Ly $\alpha W_{r}$ appears in Figure 7, coded by galaxy type, and plotted versus galaxy impact parameter (top panels) and $R_{\text {vir }}$ (bottom panels). Three features in this figure are notable. First, there is a high degree of overlap between the red and blue subsamples in terms of $W_{r}$ alone, with most values scattered between 500 and $2000 \mathrm{~m} \AA$, indicating a strong overlap between the $\mathrm{H}$ I properties of star-forming and passive galaxies (Thom et al. 2012). Second, we have detected three damped systems in the main sample; these are visible as the three strongest $W_{r}$ points. Third, the four non-detections marked here are all passively evolving galaxies. ${ }^{16}$ These four nondetections in the passive galaxies constitute a suggestive hint of a difference between the two kinds of galaxies. The gap between the main trend above $\sim 500 \mathrm{~m} \AA$ and the lower points in Figure 7 indicate this possible difference. To assess this, we use the Wilson score interval to estimate the underlying binomial hit rate above a $W_{r}$ threshold of $200 \mathrm{~m} \AA$. The 28 of 28 star-forming galaxies yield $f_{\text {hit }}=96 \pm 4 \%$, while the 12 of 16 passive galaxies give $f_{\text {hit }}=70 \pm 20 \%$ (95\% confidence limit). ${ }^{17}$ There is some indication simply in the hit rates that early type galaxies (ETGs) show H I less frequently, but uncertainty related to the sample size prevents a strong conclusion.

Simple linear fits to $\log W_{r}$ versus impact parameter are shown in Figure 7, excluding non-detections and damped systems. The star-forming subsample shows a trend with slope

\footnotetext{
16 There is one system, associated with galaxy J0943+0531 216_61, which is detected strongly in $\operatorname{Ly} \beta$ but has its $\operatorname{Ly} \alpha$ profile blended with the $\operatorname{Ly} \beta$ profile from another system at $z=0.356$ (Thom et al. 2011). This system counts as a detection of $\mathrm{H} \mathrm{I}$ in association with a passive galaxy, but it does not appear in any $W_{r}$ analysis based on $\operatorname{Ly} \alpha$. We use $\operatorname{Ly} \beta$ to estimate its kinematic extent. 17 There is ambiguity for the system 270_40 toward J2257+1340, where two small star-forming galaxies appear to lie closer to the sightline than the canonical passive galaxy. Were we to adopt a "closest" rather than "most massive" galaxy association rule (Section 2.4), these "hit rates" would be $28 / 29$ for star-forming galaxies and $12 / 15$ for passive galaxies, well within the errors quoted for these subsamples.
} 

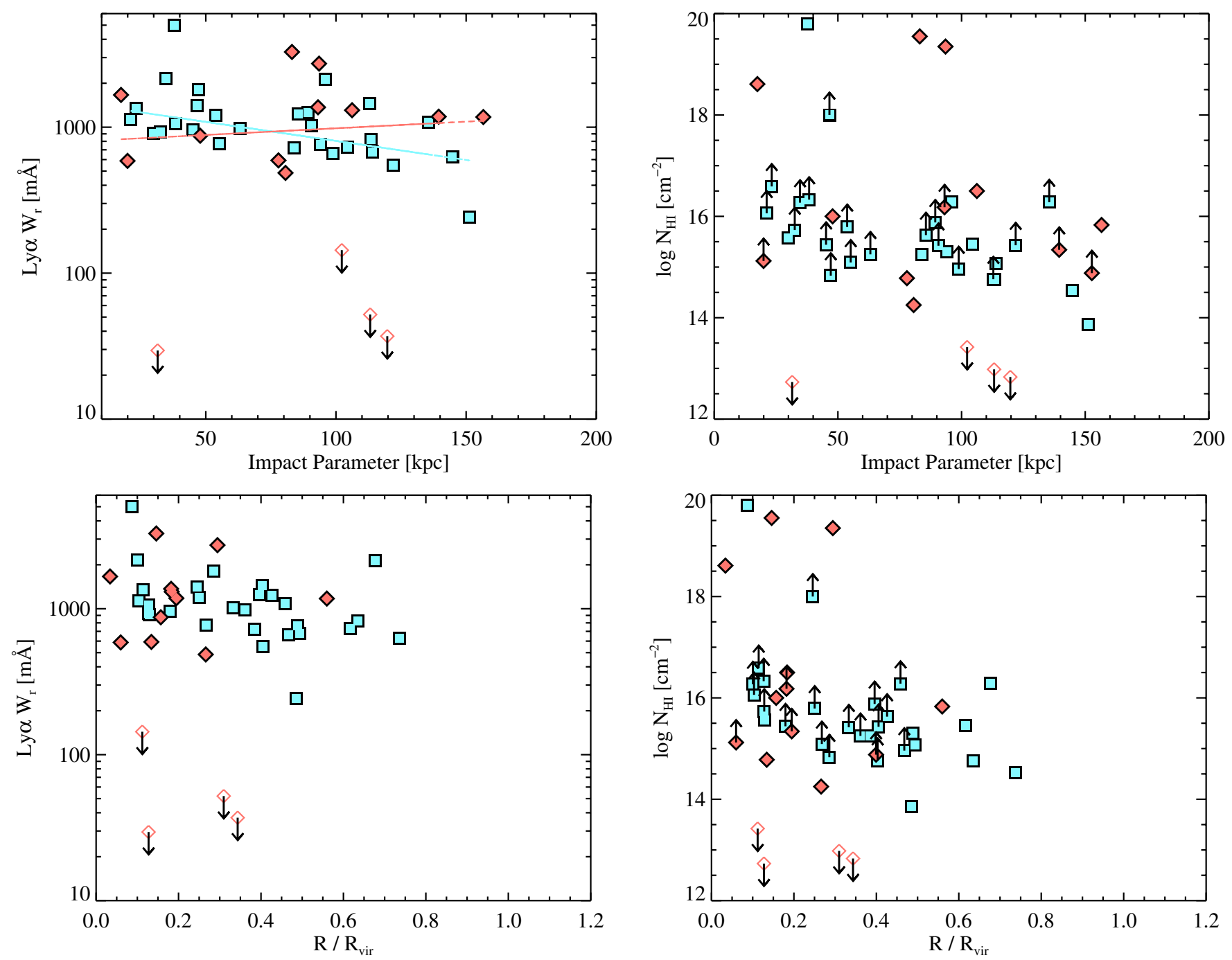

Figure 7. H I Ly $\alpha$ rest-frame equivalent width $W_{r}$ (left) and column density $N_{\mathrm{HI}}$ (right) vs. impact parameter (top) and $R_{\text {vir }}$ (bottom). Typical errors are of the same order as the symbol size. The left panels show two fits to the (non-damped) detections in the star-forming and passive subsamples. The slopes are marginally different; the apparent strengthening of the red line is still consistent with a flat slope given the small sample size.

(A color version of this figure is available in the online journal.)

$d \log W_{r} / d R=-0.0026 \pm 0.0008$. The passive subsample shows a slight strengthening at larger impact parameter, but this is not statistically significant: the slope is $d \log W_{r} / d R=$ $0.0009 \pm 0.0015$. This mild inconsistency between the two subsamples could still be a result of small sample size-the two strong detections at $\sim 150 \mathrm{kpc}$ are the chief cause of the flattened slope in the passive subsample.

If we consider the "hit rate" of damped systems, we find that $f_{\text {damped }}=9 \pm 8 \%$ ( 1 in 28 ) for the star-forming galaxies and $f_{\text {damped }}=20 \pm 16 \%$ ( 2 in 16 ) for the passive galaxies $(95 \%$ confidence). This comparison is made even more ambiguous by the presence of a star-forming galaxy at the same redshift as the canonical (and more massive) passive galaxy J0925+4004 196_22, with only a $15 \mathrm{kpc}$ separation, and by the presence of two star-forming galaxies at the same redshift as the canonical passive galaxy J0928+6025 110_35. Thus we cannot draw strong conclusions based on these small samples, but the damped systems are plausibly associated with galaxies of either type.

We can use one- and two-sample nonparametric statistics to assess the differences between the star-forming and passive subsamples in terms of Ly $\alpha W_{r}$. We use the Kaplan-Meier estimator (Feigelson \& Nelson 1985) to derive the mean $W_{r}$ and the error in the mean. If we include all 28 star-forming and 15 passive galaxies in these tests (216_61 has no clean Ly $\alpha$ measurement), we find $\left\langle W_{r}\right\rangle<1033 \pm 250 \mathrm{~m} \AA$ for the passive galaxies and $1200 \pm 260 \mathrm{~m} \AA$ for the star-forming galaxies (onesided censoring makes the passive value an upper limit). Taking the two subsamples in a two-sided Kolmogorov-Smirnov (K-S) test, ${ }^{18}$ we find that we can reject the null hypothesis that the two subsamples are drawn from the same parent distribution at $93 \%$ confidence $(D=0.395)$. However, a closer look shows that this test is affected by the presence of the four passive upper limits on one side and by the damped systems on the other. If we exclude the damped systems, the probability of null rejection increases to $96 \%$, with mean values $730 \pm 160 \mathrm{~m} \AA$ and $970 \pm 70 \mathrm{~m} \AA$ for passive and star-forming. So, apart from the damped systems, there is a suggestive indication of a difference between the two subsamples.

However, it appears that a detection or non-detection of $\mathrm{H}_{\mathrm{I}}$ is almost an either-or proposition in the passive subsample, with a

\footnotetext{
18 In this test, the passive upper limits are taken as values - the presence of all these on one side of the full distribution means their exact values between those plotted and zero do not affect the cumulative probability distribution from which the K-S statistic is computed.
} 

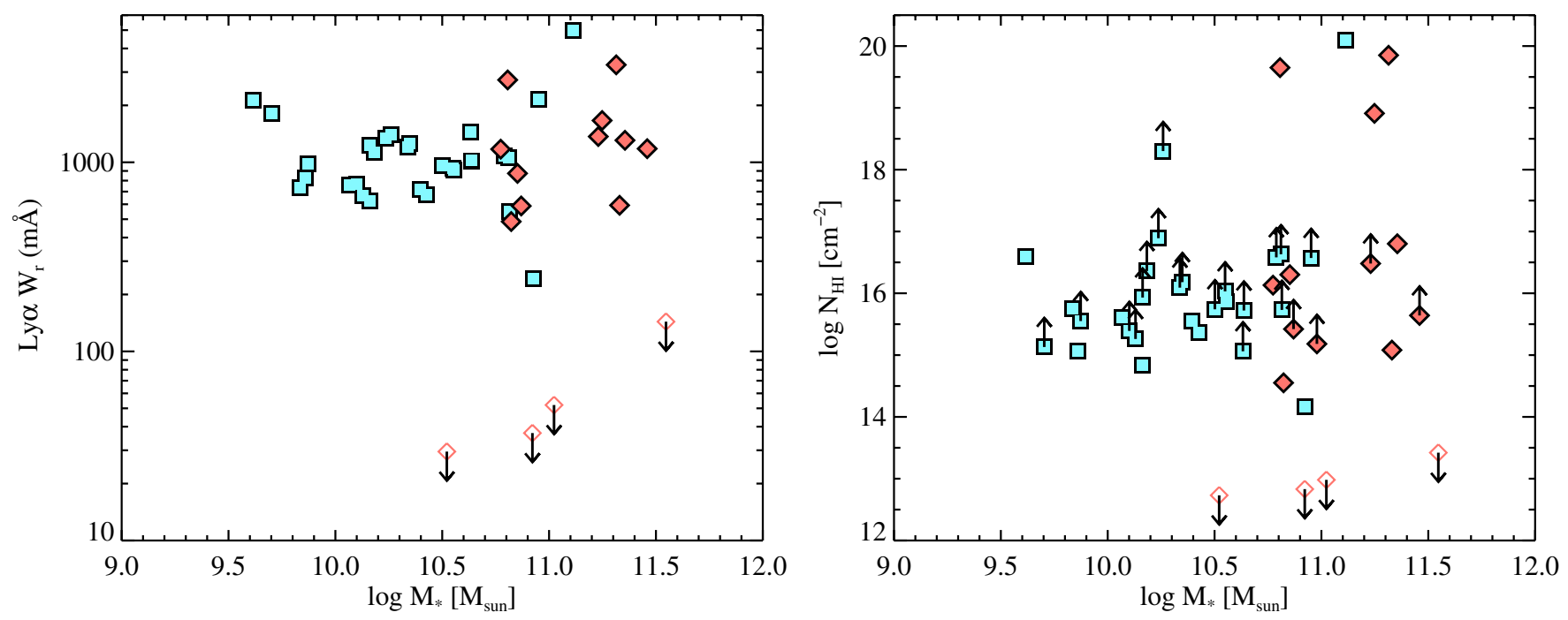

Figure 8. H I Ly $\alpha$ rest-frame equivalent width $W_{r}$ (left) and column density $N_{\mathrm{H}_{\mathrm{I}}}$ (right) vs. stellar mass $M_{*}$.

(A color version of this figure is available in the online journal.)
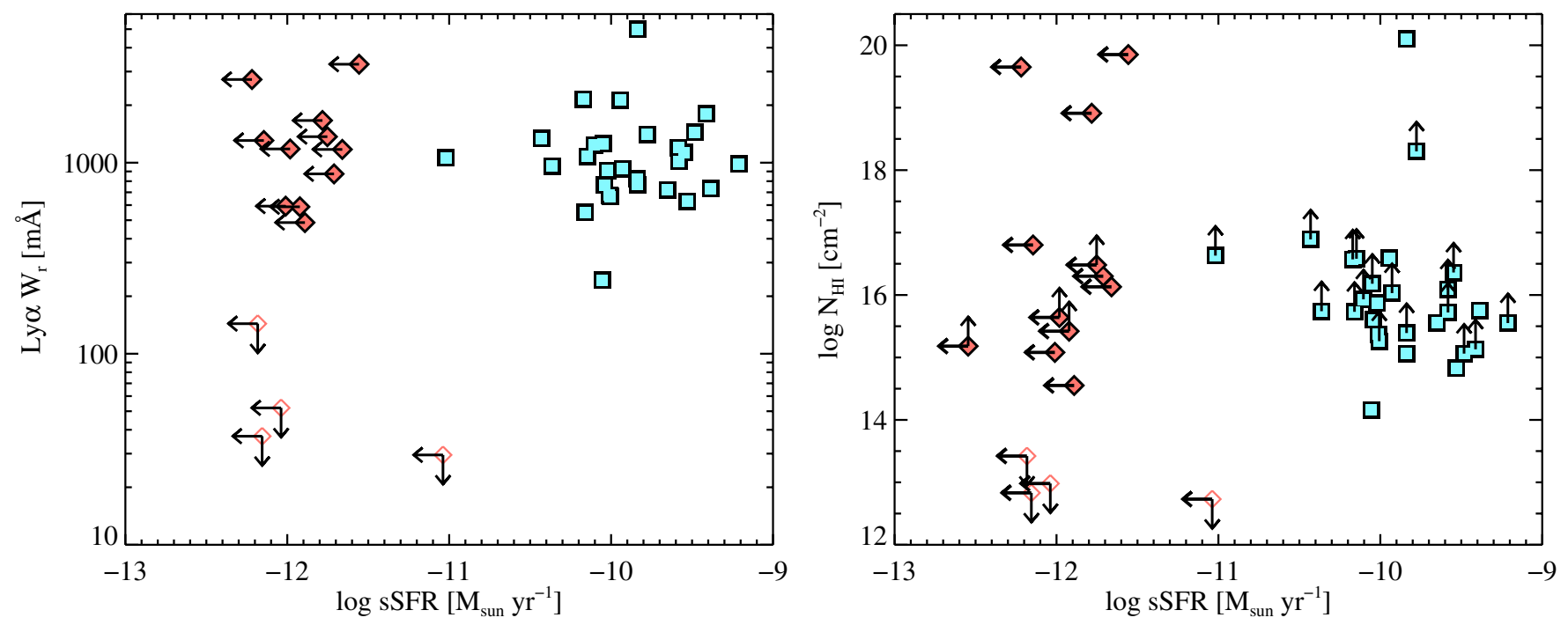

Figure 9. Ly $\alpha$ equivalent widths vs. sSFR (left). H I column density vs. sSFR (right).

(A color version of this figure is available in the online journal.)

distribution of $\operatorname{Ly} \alpha$ strengths in the detections that is similar to the star-forming subsample and well-separated from the upper limits. It may be that $\mathrm{H}$ I around passive galaxies, when it occurs, is very similar in strength to the ubiquitous detections in the star-forming subsample. To assess this case, we repeated the tests above, this time excluding both the damped systems and the upper limits in the passive subsample. In this case, we can only reject the same-parent null hypothesis at $62 \%$ confidence, and we find that the mean $W_{r}=1030 \pm 140 \mathrm{~m} \AA$ for passive and a slightly lower $W_{r}=970 \pm 70 \mathrm{~m} \AA$ for the star-forming galaxies. While the non-detection of $\mathrm{H}_{\mathrm{I}}$ in $25 \%$ of the passive galaxies does indicate a difference in the hit rates, we have no reason to conclude that, when it occurs, it is any weaker than in star-forming galaxies.

In light of all these comparisons, we conclude that there is only weak evidence of a difference in the frequency of H I absorption surrounding star-forming and passive galaxies. Once we remove the small number of non-detections, the two subsamples are not significantly different in terms of $\mathrm{H} \mathrm{I}$ strength. Because the column densities $N_{\mathrm{HI}}$ contain both left and right censoring (both upper limits for non-detections and lower limits caused by saturation), we have not attempted these non-parametric statistics for $N_{\mathrm{H}_{\mathrm{I}}}$. It remains possible that there is a difference in the intrinsic $N_{\mathrm{H}}$ distributions of the two subsamples that is masked by saturation. In other words, it is possible that one of the subsamples has a systematically higher average $N_{\mathrm{H} \text { I }}$ (or shallower power law distribution of column density, $f_{\mathrm{HI}_{\mathrm{I}}}$ ), which has gone undetected because of saturation effects on the flat part of the curve-of-growth. Another confusing factor is that the lower limits on saturated $N_{\mathrm{H} \text { I }}$ depend mainly on the unrelated redshift of the system through the availability of the Lyman series lines (see Figure 6). Thus the actual values could be higher, on average, than the formal lower limits for one of the subsamples and still go unseen.

We can also examine the properties of the detected $N_{\mathrm{HI}}$ in comparison to the stellar mass $M_{*}$ and SFR. Figure 8 shows $W_{r}$ and $N_{\mathrm{HI}}$ versus $M_{*}$ and Figure 9 shows these in comparison to the specific star formation rate $\left(\mathrm{sSFR} \equiv \mathrm{SFR} / M_{*}\right)$. Here the slight preference of the low values for the passive galaxies 

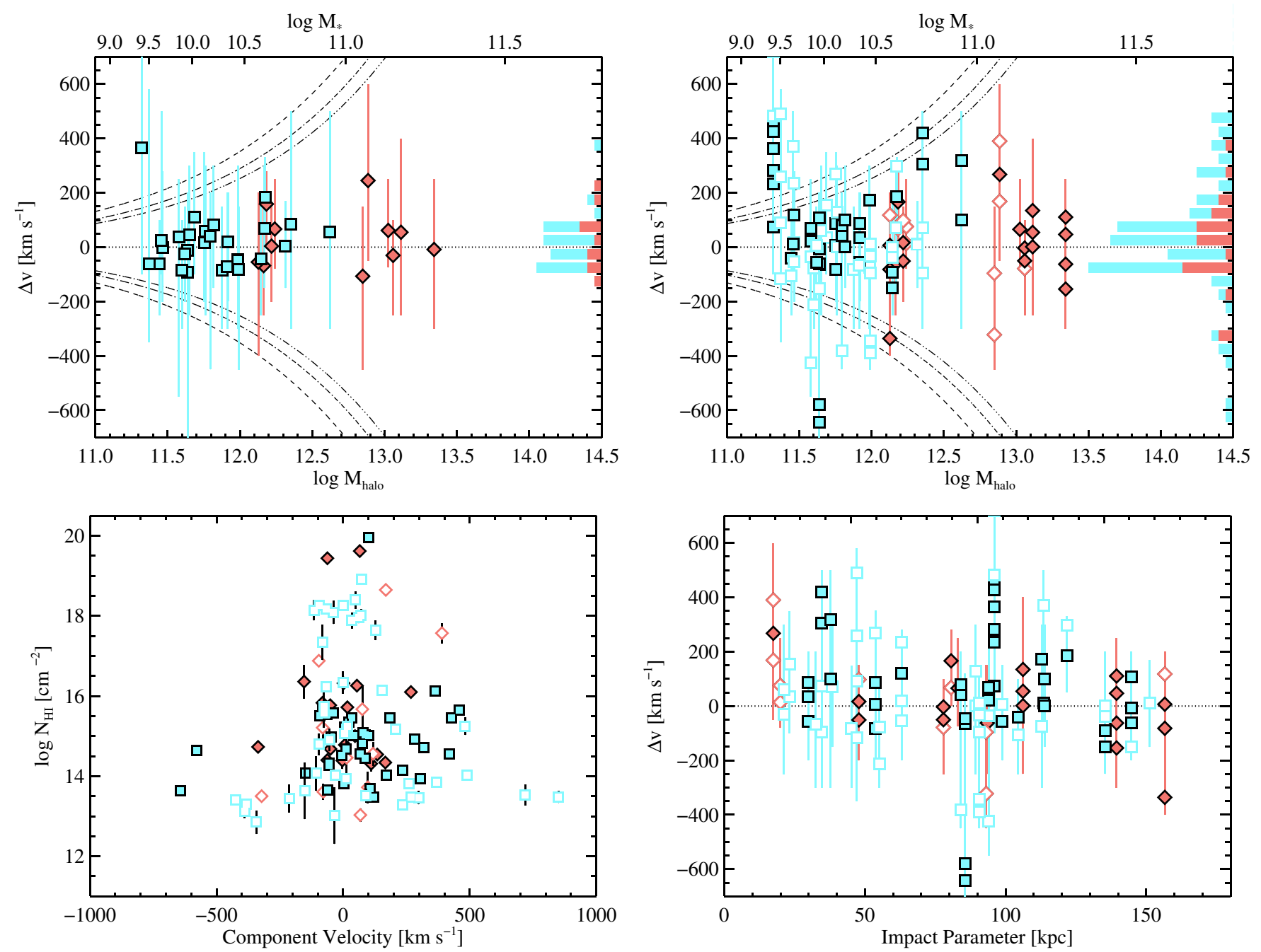

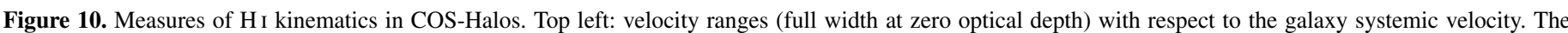

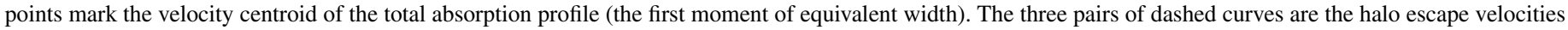

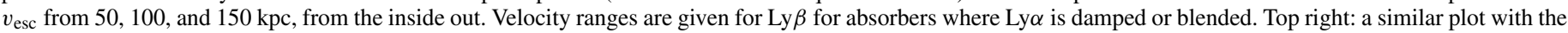

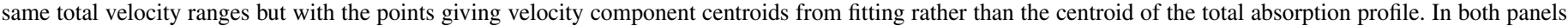

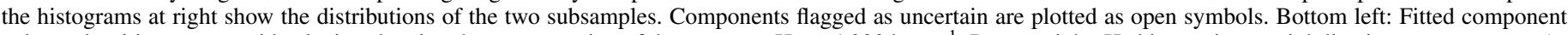

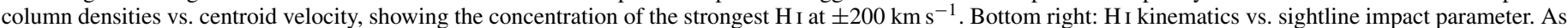
in the panel at top right, the extent of the solid lines marks the full velocity width at zero optical depth, while the symbols mark the centroids of fitted components.

(A color version of this figure is available in the online journal.)

can be readily seen, but we have already judged it to be of marginal statistical significance. There are no apparent trends of absorption strength with either stellar mass or sSFR. From the right panel of Figure 8 it appears that all three damped systems have $\log M_{*}>10.5$. While the number is small, we speculate that this reflects the increasing chance of the sightline through a massive galaxy halo passing near a gas-rich satellite that might give rise to a damped absorber (the target galaxies themselves are $\gtrsim 40 \mathrm{kpc}$ from the sightline, so that the target themselves are less likely to host the damped absorption). Conversely, the damped systems found serendipitously in the COS-Halos data (Battisti et al. 2012) do not appear to have luminous galaxies nearby. This suggests that at least some DLAs are associated with sub- $L^{*}$ or low surface-brightness galaxies. It is also notable that the increased frequency of damped systems occurs in the same range of stellar mass as the four non-detections. Thus the scatter in $N_{\mathrm{H}_{\mathrm{I}}}$ in this region is much larger than below $\log M_{*} / M_{\odot}=10.5$. We speculate that this large scatter reflects a greater diversity of origins for the detected gas, in contrast with the lower mass range where the detected gas follows a narrower trend. However, as demonstrated above these sample sizes are too small to draw a robust conclusions; more data above $\log M_{*} \sim 10.5$ is needed to assess whether damped absorbers and non-detections have higher covering fractions in these halos as the host mass increases, and to identify these trends with particular origin scenarios.

\subsection{H I Kinematics}

In addition to line strengths and column densities, we can also examine the kinematics of the $\mathrm{H}$ I absorption and its distribution across the galaxy subsamples. Measures of absorber kinematics with respect to galaxies appear in Figure 10. First, we examine $\Delta v$, the full velocity extent of the detected absorption at zero optical depth (marked as solid lines in the figures), using the velocity integration ranges of $\operatorname{Ly} \alpha$ as the measure. The full velocity width includes contributions from both thermal broadening and bulk flow, so it is only a proxy for constraining 

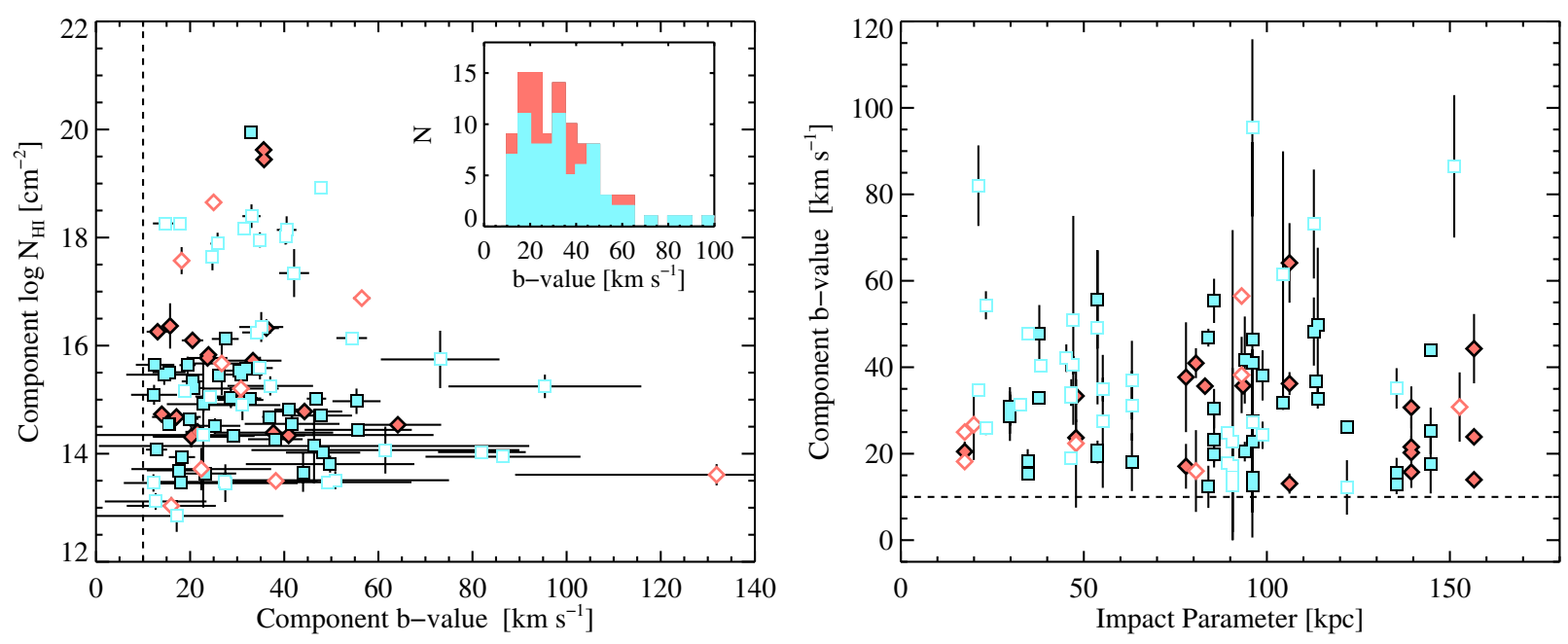

Figure 11. Left: fitted component Doppler $b$-values vs. $N_{\mathrm{H}_{\mathrm{I}}}$ for profile-fitted components. Systems with uncertain fits owing to saturation, ambiguous placement of components, or data quality issues are flagged as such in Table 5 and plotted with open symbols here. Generally speaking, component column densities are uncertain above $\log N_{\mathrm{H}_{\mathrm{I}}} \simeq 16$ owing to saturation in even the highest available Lyman lines and remain so until damping wings reappear at $\log N_{\mathrm{H}_{\mathrm{I}}} \simeq 18.5$. The main uncertainty in the fitted $b$-values arises from properly assigning the number of components in a strong profile; given limited resolution, $\mathrm{S} / \mathrm{N}$, and blending it is likely that the number of components is underestimated and so the fitted $b$ here are overestimates. The components with $b \gtrsim 80 \mathrm{~km} \mathrm{~s}^{-1}$ are not robustly constrained at the S/N of the data. Some weaker components, $\log N_{\mathrm{H}} \lesssim 14$, are considered uncertain because of noise or possible contamination. The inset histogram shows the distribution of the Doppler $b$ for both subsamples together. Right: fitted component linewidths vs. impact parameter with the same symbol coding.

(A color version of this figure is available in the online journal.)

the maximum kinematic extent of the absorbing gas. ${ }^{19}$ We also use the velocity centroid of the detected absorption, measured as the first moment of equivalent width. Recall that these velocities are measured in the rest-frame of the galaxy systemic redshift, with $z_{\text {sys }}$ identified with $v=0$. Here we find that most of the detected $\mathrm{H}$ I absorption is located within $\pm 200 \mathrm{~km} \mathrm{~s}^{-1}$ of the galaxy. There are exceptions, with some systems showing a total extent of $\gtrsim 500 \mathrm{~km} \mathrm{~s}^{-1}$, including such notable systems as the "cold accretion" absorber toward J0943+0531 (Thom et al. 2011). There are also some strong components in other systems and a few weaker "Ly $\alpha$ only" components (see Table 5) at $|v|>200 \mathrm{~km} \mathrm{~s}^{-1}$. However, statistically the data argues for concentration within $\pm 200 \mathrm{~km} \mathrm{~s}^{-1}$; all but two centroids out of 40 detected systems lie within $\pm 200 \mathrm{~km} \mathrm{~s}^{-1}$. This concentration is seen clearly in the other three panels of Figure 10, which use the velocity centroids of fitted components. In the upper right panel, we see that, by number, $74 \%$ of all detected components lie within $\pm 200 \mathrm{~km} \mathrm{~s}^{-1}$ of the systemic velocity and $81 \%$ are within $\pm 300 \mathrm{~km} \mathrm{~s}^{-1}$. By contrast, only $9 \%$ of detected components lie between 400 and $600 \mathrm{~km} \mathrm{~s}^{-1}$ away from the systemic velocity.

The lower left panel shows the column-density dependence of the component velocity distribution. Here we see that component fits implying high column density concentrate more strongly at low velocity than weaker components. While the column densities of the strongest components are made uncertain by saturation, there is no systematic effect preventing high $N_{\mathrm{H} \text { I }}$ components from appearing at higher velocities, so this is a real effect. Counting by column density produces an even stronger concentration than counting by number: $90 \%$ of the total fitted column density lies within $\pm 100 \mathrm{~km} \mathrm{~s}^{-1}$, while $99.8 \%$ lies within $\pm 200 \mathrm{~km} \mathrm{~s}^{-1}$ and only $0.2 \%$ is outside that range (this excludes the damped systems, which all have $v<100 \mathrm{~km} \mathrm{~s}^{-1}$ and so would only reinforce the trend toward low velocities, but

\footnotetext{
19 For systems with $\log N_{\mathrm{H}} \gtrsim 18.5$, the Ly $\alpha$ width reflects damping wings, not kinematic broadening. Also, galaxy 216_61 toward J0943+0531 is

detected in $\operatorname{Ly} \beta$ but contaminated at $\operatorname{Ly} \alpha$. For those systems, we substitute the full width at zero optical depth of $\operatorname{Ly} \beta$ for this test.
}

would unfairly dominate the total column density). It is also notable that, while there are weak $\log N_{\mathrm{H} \text { I }} \lesssim 15$ components at all relative velocities, the high-velocity components tend strongly to have these lower column densities. The lower right panel of the figure shows that the full velocity range of the absorption and the velocity centroids of fitted components do not exhibit any clear trends with impact parameter (the full width at zero optical depth is still marked in solid lines).

These velocities are of the same order as the velocity dispersions of the galaxies, and so are consistent with a gravitational association between the galaxies and the gas. Figure 10 also compares these velocity ranges (top left panel) and the velocities of fitted components (top right panel) to the inferred escape velocity of the host halos, with $M_{\text {halo }}$ converted from $M_{*}$ using the relation of Moster et al. (2010). We find that only a small portion of the absorption lies outside the inferred $v_{\text {esc }}$, especially above $\log M_{\text {halo }} \simeq 12$. Thus there is scant evidence that the bulk of the detected $\mathrm{H}$ I is able to escape the host galaxy. It is possible that there is escaping material outside $\pm 200 \mathrm{~km} \mathrm{~s}^{-1}$ that is too weak to detect at the $\mathrm{S} / \mathrm{N}$ ratio of our survey, or that substantial material escaped at earlier epochs of galaxy evolution, but the bulk of the detected H I (and, by inference, the mass budget that it traces) is not escaping at the time we observe it. These results could mean that the CGM we detect is essentially bound to the host galaxies. Furthermore, its kinematics have no apparent dependence on galaxy type.

Finally, we note that the full linewidths of the absorbers as shown in Figure 10 generally range over $\pm 100-200 \mathrm{~km} \mathrm{~s}^{-1}$ and center near zero; because they depend weakly on $N_{\mathrm{HI}}$, the total profile linewidth is a poor measure of total column density or internal kinematics and should be treated cautiously in lowresolution or low-S/N data.

\subsection{H I Linewidths and Temperatures}

Another important test is the internal kinematics of the detected absorption as represented by the Doppler $b$ parameters of the fitted components. Figure 11 shows the fitted $N_{\mathrm{H}_{\mathrm{I}}}$ and $b$ for all components without grouping them into their host systems. 
We see that almost all the absorption lies in systems with $b<60 \mathrm{~km} \mathrm{~s}^{-1}$, while more than half (by component number, not column density) has $b \lesssim 40 \mathrm{~km} \mathrm{~s}^{-1}$. We can interpret these Doppler $b$ parameters as robust upper limits to the temperature, if we adopt $b=\sqrt{2 k T / m_{\mathrm{H}}}$. Thus almost all the detected H I has $T \lesssim 220,000 \mathrm{~K}\left(60 \mathrm{~km} \mathrm{~s}^{-1}\right)$ and most have $T \lesssim 100,000 \mathrm{~K}$ $\left(40 \mathrm{~km} \mathrm{~s}^{-1}\right)$. These temperatures are significantly below the implied virial temperatures of the host halos, which are 1 million $\mathrm{K}$ or more for galaxies with $M_{\text {halo }} \gtrsim 10^{12} M_{\odot}$.

There are two reasons why these values are robust upper limits to $b$ and $T$. First, our method for fitting decomposes the observed profiles into the minimum number of components necessary to achieve a good fit; the actual number of components could be larger, which would reduce the typical $b$ and thus the implied temperatures. Second, the values quoted above assume that the observed line broadening is purely thermal, which maximizes the implied temperatures. With non-thermal motions of $30 \mathrm{~km} \mathrm{~s}^{-1}$, the implied temperatures for $b=40$ and $60 \mathrm{~km} \mathrm{~s}^{-1}$ drop to 40,000 and $160,000 \mathrm{~K}$, respectively. ${ }^{20}$ Finally, recall that components with uncertain fits owing to saturation, component placement, or poor significance are marked with open symbols in the plots.

Figure 11 also shows that there is no discernible difference in line widths between the star-forming and passive subsamples. A $\mathrm{K}-\mathrm{S}$ test on the red and blue distributions in the inset histogram returns a K-S statistic of $D=0.164$ and a null rejection probability $P=0.64$ for 26 red and 73 blue components. We conclude there is no evidence for a difference in line widths in the two subsamples. This corresponds to no significant difference in the inferred temperature limits, and no difference in the temperatures themselves if the relative contributions of nonthermal broadening are the same.

\section{COS-HALOS COMPARED TO PREVIOUS STUDIES OF IGM/CGM H I AND GALAXIES}

\subsection{Correlation with Radius: Comparisons of H I Strength}

Surveys of Hi absorbers in the spectra of QSOs have been pursued fruitfully with every generation of spectrographic capability on $H S T$, starting with the Key Project on QSO absorption-line systems using FOS (Bahcall et al. 1993; Jannuzi et al. 1998) and the first pioneering high-resolution studies with GHRS by Morris et al. (1991). Investigators using data from the Key Project and follow-up galaxy redshift surveys firmly vindicated the early prediction by Bahcall \& Spitzer (1969) that diffuse gas in galaxy halos would imprint absorption lines in the spectra of distant QSOs. This work established the existence of an extended, diffuse gaseous medium surrounding galaxies out to hundreds of kpc, traced mainly by Ly $\alpha$ (Morris et al. 1993; Lanzetta et al. 1995). Further studies that added images of the galaxy fields and additional spectroscopy were able to examine the relation of this gaseous halo to galaxy type and mass (proxied by luminosity). Chen et al. (1998) reported that the physical extent of the Ly $\alpha$ CGM scaled slowly with $B$ and $K$ band luminosity, such that more massive galaxies have more extended gaseous halos. A contemporaneous study by Tripp et al. (1998) used more sensitive absorption-line data to examine the galaxy correlations of weaker $\operatorname{Ly} \alpha$ absorbers with a smaller sample of galaxies, finding that even absorbers with $<100 \mathrm{~m} \AA$ are not randomly distributed with respect to galaxies.

\footnotetext{
20 The $R \sim 18-20,000$ resolution of COS means that we cannot reliably fit linewidths below $b \simeq 10 \mathrm{~km} \mathrm{~s}^{-1}$. Fits to broader lines can be affected by the non-Gaussian wings of the COS LSF, for which we include a detailed model.
}

In a series of papers based on GHRS/G160M data, the Colorado IGM group examined a set of $\sim 100$ Ly $\alpha$ absorbers (Penton et al. 2000b) and studied their correlations with galaxies from published surveys, finding that the stronger absorbers cluster more weakly with galaxies than galaxies cluster with themselves, while the weakest absorbers are even more randomly distributed and so do not lie in individual galaxy halos but arise instead in the LSS of filaments (Penton et al. 2002). This tendency for the strongest $\mathrm{H}_{\mathrm{I}}$ to appear near galaxies and for weaker $\mathrm{H}$ I to arise in larger, more diffuse structures such as cosmic web filaments in also seen in hydrodynamical simulations at low redshift (Davé et al. 1999) and at high redshift (Kollmeier et al. 2003), making it clear that even absorption that appears to lie very near galaxies $(\lesssim 100 \mathrm{kpc})$ may in fact arise further out in extended LSSs ( $\gtrsim 1 \mathrm{Mpc}$ ), particularly at low column densities. Taking all low-redshift $\mathrm{H}$ I clouds together, Penton et al. (2000a) estimated that Ly $\alpha$ clouds contain approximately $20 \%$ of the cosmic baryons at low redshift. Their later study using STIS extended this analysis to weaker systems and brought the baryonic mass fraction up to $29 \%$ of the cosmic value (Penton et al. 2004).

Using cross-correlation methods on a single sightline, Chen et al. (2005) confirmed that even weak Ly $\alpha$ correlates with galaxies out to $\sim 1 \mathrm{Mpc}$, particularly for star-forming galaxies. With STIS, Bowen et al. (2002) further characterized the Ly $\alpha$ surrounding galaxies by pre-selecting galaxies prior to obtaining the absorption data, as COS-Halos has done. Prochaska et al. (2011b) followed up absorber samples from HST and FUSE after compiling galaxy redshifts for these sightlines, but did not select the sightlines themselves based on the availability of foreground galaxies. They reported a strong H I-traced CGM out to $\simeq 300 \mathrm{kpc}$, with no strong dependence on galaxy type but with some preference of the highest $W_{r}$ systems to be associated with galaxies at $L \gtrsim L^{*}$. Surveying a large compilation of data on galaxies of $z<0.017$ and absorbers from HST and FUSE, Wakker \& Savage (2009) confirmed this basic conclusion. Thus the existence of a diffuse, ionized medium surrounding galaxies, with an extent weakly depending on galaxy properties, was well established before the advent of COS. Recently, Stocke et al. (2013) have revisited this issue with a new sample of targeted galaxies and serendipitous QSO-galaxy pairs drawn from earlier studies. They report that CGM clouds within $\sim 0.4 R_{\text {vir }}$ of galaxies tend to be "warm"- at photoionized temperatures - metal enriched $\left(Z \sim 0.1-1 Z_{\odot}\right)$, and probably bound.

Our goal now is to compare the COS-Halos results to these prior studies to test for similarities and differences in the gas surrounding galaxies, mainly as a function of impact parameter. Because of its selection technique, COS-Halos covers a range of impact parameter that is lower than the typical radius probed by the previous studies of this size. Our sample is about twice the size of Chen et al. (2001) out to $150 \mathrm{kpc}$, while Prochaska et al. (2011b) contains only three $L \sim L^{*}$ galaxies with $\rho \lesssim 150 \mathrm{kpc}$. Thus COS-Halos is well-positioned to study the dependence of the CGM on galaxy type and/or mass, while it is less useful for probing the outer regions of the CGM ( $\left.R \gtrsim 0.5 R_{\mathrm{vir}}\right)$. To test for evolution as a function of impact parameter, we need samples at $\gtrsim 300 \mathrm{kpc}$, for which many of these other studies are well-suited.

However, this goal presents the immediate problem that these various studies used a wide range of selection techniques and analysis methods. The COS-Halos galaxies (15-160 kpc) were selected for study without any knowledge of the absorption; this 
is true as well of the pioneering study of six targeted galaxies by Bowen et al. (2002) and of the "targeted" portion of the COS sample of Stocke et al. (2013). The other surveys are "blind," in the sense that they are based on absorber data from sightlines that were not chosen to probe particular galaxies, and the galaxy data was compiled separately and then correlated with the absorbers. We emphasize that these studies are heterogeneous in many respects; they use different criteria for absorber and galaxy selection, different sources of galaxy redshifts and photometry from custom follow-up spectroscopy (e.g., Chen and Prochaska) to literature galaxy catalogs (e.g., Penton and Wakker). Most importantly for comparison with COS-Halos, each survey has used different rules for associating galaxies and absorbers in physical and velocity space. Moreover, as many of these surveys are based on the common body of HST spectroscopic data, they overlap in the sightlines and redshift intervals that they cover, and so have many individual absorber/galaxy pairs in common. They are thus not independent surveys in terms of the objects studied. Rather than attempt the daunting task of reconciling all these disparate studies, or combining overlapping samples, instead we take these prior studies as independent empirical characterizations of the $\mathrm{H}$ I gas surrounding galaxies as seen by different groups using different techniques and analysis criteria at different times.

Figure 12 compares the COS-Halos results to these other studies in terms of Ly $\alpha$ equivalent width $W_{r}$ versus the impact parameter at $<1 \mathrm{Mpc}$. Upper limits for galaxies near the sightlines with no corresponding absorption are shown for Tripp et al. (1998), Chen et al. (2001), and Prochaska et al. (2011b) and COS-Halos.

There is a clear trend for Ly $\alpha$ detections to increase in strength within $\sim 200 \mathrm{kpc}$, while outside that impact parameter range the detections are weaker, with large scatter. This trend is consistent with the conclusion that absorption found within $150 \mathrm{kpc}$ of galaxies by COS-Halos is associated with the selected galaxies and not (generically) with gas at larger distances away from the galaxies (see Section 6.2 below for more on this point). This interpretation is supported by the kinematic associations of strong H I with galaxies presented above in Section 4.2. Within the region they have in common, COS-Halos and the samples from Bowen, Chen, and Prochaska agree well considering only detection strength.

The samples from COS-Halos, Bowen, Stocke, and Prochaska provide the closest possible comparisons, since they all use galaxies predominantly selected or cataloged prior to the absorber data. For these samples the evolution from $\lesssim 150 \mathrm{kpc}$ to $\gtrsim 300 \mathrm{kpc}$ is clear. Over the range $100-200 \mathrm{kpc}$ there is apparent disagreement in the median equivalent widths between COS-Halos on the one hand and Wakker \& Savage and Penton on the other; the latter two agree with each other well. This may be caused by a difference in the selection technique or the typically lower luminosity of the galaxies in the latter two samples. The lower average redshifts in the latter two samples may also play a role if the CGM evolves to lower column density at lower redshift.

Statistical tests of the observed trends with impact parameter provide another argument for the containment of the detected H I within the virial radius. As such a test, we compare our empirical distributions of $W_{r}$ against those of Prochaska et al. (2011b), which was cut for $L>L^{*}$ galaxies and contains mostly points outside $200 \mathrm{kpc}$ (see Figure 12). Accounting for censoring in a two-sample generalized Wilcoxon test, we find that we can reject at $>99.7 \%$ confidence the null hypothesis
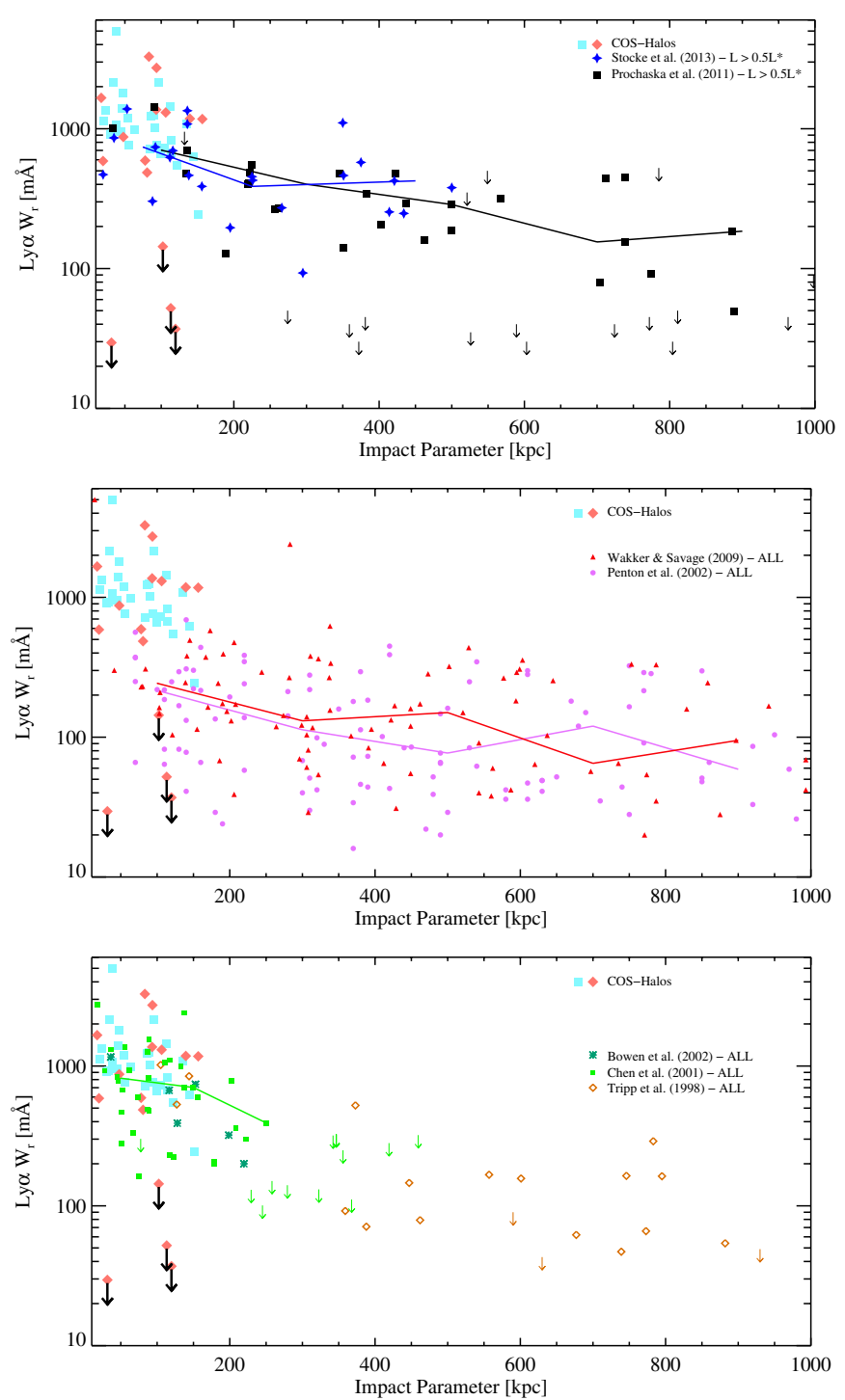

Figure 12. Comparison of $\mathrm{H}$ I strength with respect to galaxies from COS-Halos and seven previous studies. The points from Tripp et al. (1998), Chen et al. (2001), and Prochaska et al. (2011b) include upper limit arrows for galaxies in their surveys without detected absorption at the level indicated. The Prochaska et al. (2011b) and Stocke et al. (2013) samples have been cut for $L>0.5 L^{*}$ to approximate the selection criteria for COS-Halos. When present, the solid trend lines show the median $W_{\text {Lya }}$ for detections in the indicated bins for samples large enough to give meaningful binned results ( 5 points per $200 \mathrm{kpc}$ bin).

(A color version of this figure is available in the online journal.)

that the two $W_{r}$ distributions are drawn from the same parent population ( $P=0.003$ or less). If we restrict the Prochaska et al. (2011b) sample to $\rho<500 \mathrm{kpc}$, this confidence drops to $>99 \%$ but is still highly significant. These probabilities are $<10^{-5}$ for the Wakker \& Savage and Penton samples, allowing us to reject the null hypothesis at high confidence. Alternatively, if we compare to the Chen, Bowen, and Stocke samples by restricting them to the $<160 \mathrm{kpc}$ region surveyed by COS-Halos, we find that the rejection probabilities drop to $60-70 \%$, indicating highly overlapping samples and no significant evidence of disagreement in Ly $\alpha$ strength. Thus the surveys concentrated at $<200 \mathrm{kpc}$ agree with one another well, but not with those surveys concentrated outside $200 \mathrm{kpc}$. Based on these results we conclude that there is significant evidence of evolution in $\mathrm{H}$ I between the $<200 \mathrm{kpc}$ region near galaxies and further out. On the basis of these simple models and empirical 
characterizations with respect to impact parameter, we conclude that the $\mathrm{H}$ i detected by COS-Halos and these other surveys is concentrated within the $\sim 300 \mathrm{kpc}$ region surrounding galaxies. However, there remains a strong likelihood that the equivalent widths observed in COS-Halos receive a contribution from foreground and/or background gas at $\gtrsim 300-1000 \mathrm{kpc}$, up to the $\simeq 100-300 \mathrm{~m} \AA$ levels seen at these impact parameters in other samples with sightlines further away from galaxies.

Gas surrounding galaxies at high redshift has also been observed in the spectra of background QSOs using optical telescopes on the ground. A recent example is the Keck Baryon Structure Survey (KBSS; Steidel et al. 2010; Rudie et al. 2012). In their characterization of the $\mathrm{H}$ I surrounding 886 galaxies with $z \sim 2.3$ (25 of which are probed at $\leqslant 200$ physical kpc), Rudie et al. (2012) show that the strongest absorbers, $\log N_{\mathrm{H}_{\mathrm{I}}} \sim 15-17$ concentrate within $200 \mathrm{kpc}$ (physical) and $\pm 300 \mathrm{~km} \mathrm{~s}^{-1}$ of their targeted galaxies. Comparing against the low-redshift samples, they find that the covering fractions at $z \sim 2$ are similar to those measured within $300 \mathrm{kpc}$ for limits $\log N_{\mathrm{HI}} \gtrsim 14$, with $f_{c}=81 \pm 6 \%$ in the KBSS sample $(z \sim 2.3), 72 \%$ for Chen et al. (2001) $(z=0.1-0.9)$ and $70 \%$ for Prochaska et al. (2011a) $(z<0.4)$. The overall $91 \%$ detection frequency in the COS-Halos sample is consistent with these values, though higher likely because our survey region is restricted to $150 \mathrm{kpc}$. Removing the COS-Halos passive galaxies from the comparison, we find a $100 \%$ detection rate above $10^{14} \mathrm{~cm}^{-2}$, in good agreement with the $92 \pm 5 \%$ rate inside $200 \mathrm{kpc}$. Thus we conclude that the overall column density distribution and covering fractions of $\mathrm{H}$ I surrounding galaxies at the different epochs are broadly similar. We do not, however, reproduce the KBSS finding that $\mathrm{H}$ i component linewidths increase slightly inside $300 \mathrm{kpc}$ (their Figure 28 versus our Figure 11. This difference may result from the lower spectral resolution of the COS data, the smaller physical radius over which our data span, or to a real difference in the internal kinematics and/or temperature of circumgalactic gas at two different cosmic epochs. KBSS does not include detailed comparisons to galaxy properties such as mass and SFR, but the small number of $z>2$ CGM absorbers studied in connection with detailed galaxy properties reveal CGM accretion at this early cosmic epoch (e.g., Bouché et al. 2013; Crighton et al. 2013). We note also that the $\mathrm{H}$ I envelopes surrounding mainstream galaxies at all these redshifts are substantially weaker than those detected around $z \sim 2$ QSOs by Prochaska et al. (2013). We leave a more thorough and detailed comparison to be made later, when the galaxy properties for the KBSS fields have been published and we can compare against the COS-Halos findings with respect to galaxy mass and type.

\subsection{Column Density and Temperature Comparisons}

Because COS-Halos typically covers Lyman series lines above Ly $\beta$ with moderate resolution $\left(\sim 20 \mathrm{~km} \mathrm{~s}^{-1}\right)$, we can address the internal kinematics of the CGM gas in a more rigorous manner than previous studies that focused on Ly $\alpha$ alone. As discussed above, line-profile broadening takes two basic forms: thermal broadening that scales as $T^{1 / 2}$, and non-thermal broadening from, e.g., turbulence, bulk flows, or gravitational motions. If we can assess these separately, we can address whether temperature or other physical quantities differ in the CGM. To do this, we compare the line-profile fits from COS-Halos to the "blind" samples of Tripp et al. (2008), Thom \& Chen (2008), and Tilton et al. (2012). These three studies analyze (highly overlapping) samples of intervening $\mathrm{HI}_{\mathrm{I}}$ absorbers from $H S T$ and FUSE data. All have tabulated line-profile fits based on Lyman series lines for each absorber. Figure 13 shows $N_{\mathrm{H}_{\text {I }}}$ versus $b$ for COS-Halos and these three samples. The blind comparison samples have no statistically significant differences between them in terms of b-value distribution; considered pairwise, the two-sided K-S tests yield $P$ values in the range $0.15-0.92$. It is important that the comparison samples were blind sample of absorbers obtained without prior knowledge of galaxy coincidences, and have a large degree of overlap with one another. They effectively provide a measure of how different the $N_{\mathrm{H} \text { I }}$ and $b$ value distributions of the same parent sample can differ depending on the sample selection and treatment of fitting by different investigators. ${ }^{21}$ Indeed, there is no statistically significant difference between them. If we compare the COS-Halos $b$-value distribution to these samples, we find that its K-S probabilities with respect to each are in the range 0.4-0.92; in other words, there is no evidence that the COS-Halos CGM distribution is more different from the blind IGM samples than they are from each other. We find no evidence that there is significant evolution in internal kinematics and the implied temperatures inside and outside the $150 \mathrm{kpc}$ region of our survey.

Considered together with the $\mathrm{H}$ I equivalent width and kinematics shown above, we interpret these line broadening results as follows. There is a trend in the H I equivalent width at low impact parameter (Figure 7). Because the typical Ly $\alpha$ line is strongly saturated, this trend is manifested primarily as a broadening of the profile of total $\mathrm{H}$ I absorption, but component structure is usually not resolved in Ly $\alpha$. For those systems where we can use higher Lyman series lines to separate blended components in a profile fit, the fitted Doppler $b$ parameters, which imply upper limits on temperatures, fall in a distribution that is not significantly different from blind samples of intervening absorbers. The COS-Halos data indicate that that the broadening of Ly $\alpha$ absorption near galaxies owes more to the piling up of multiple absorption components and/or increases in total column density than to an apparent increase in the temperature of CGM gas (cf. Wakker \& Savage 2009). Indeed, given the inability of COS to resolve any components at $b \lesssim 10 \mathrm{~km} \mathrm{~s}^{-1}$, and the severe blending problems that impede the proper counting of components, it is possible that gas near galaxies is colder on average than further out in the IGM but that the effect is masked by blending and saturation and goes unseen. It seems that "cold" IGM/CGM clouds, wherever they are found, have roughly the same temperatures that are not determined by their locations with respect to galaxies.

However we emphasize that using $\mathrm{H}$ I as a tracer of CGM gas temperatures presents a biased view, as it preferentially detects material at $T \lesssim 10^{5} \mathrm{~K}$ where the neutral fraction of $\mathrm{HI}$ is high enough to yield detected, or even strong, absorption. There may be a hotter and/or more photo-ionized CGM component, such as that traced by high ions like O VI or Ne VIII, that contributes little to the observed H I. It is possible that some broad, weak H I absorption, which could trace a component of the CGM nearer to the expected halo virial temperatures, has gone undetected in our data. Ly $\alpha$ lines with $T=0.5-1$ million $\mathrm{K}$ and purely thermal broadening will have $b=90-130 \mathrm{~km} \mathrm{~s}^{-1}$, and detections of such "broad Ly $\alpha$ absorbers" (BLAs) suggest that they may depart from the unabsorbed continuum by only $10 \%-15 \%$, even in the line center (Savage et al. 2011b, 2011a). While some broad Ly $\alpha$ components are distinctly evident and separated from

\footnotetext{
21 We use Tilton's consensus values from their Table 5, which attempted to
} reconcile disparate measurements in this sense. 

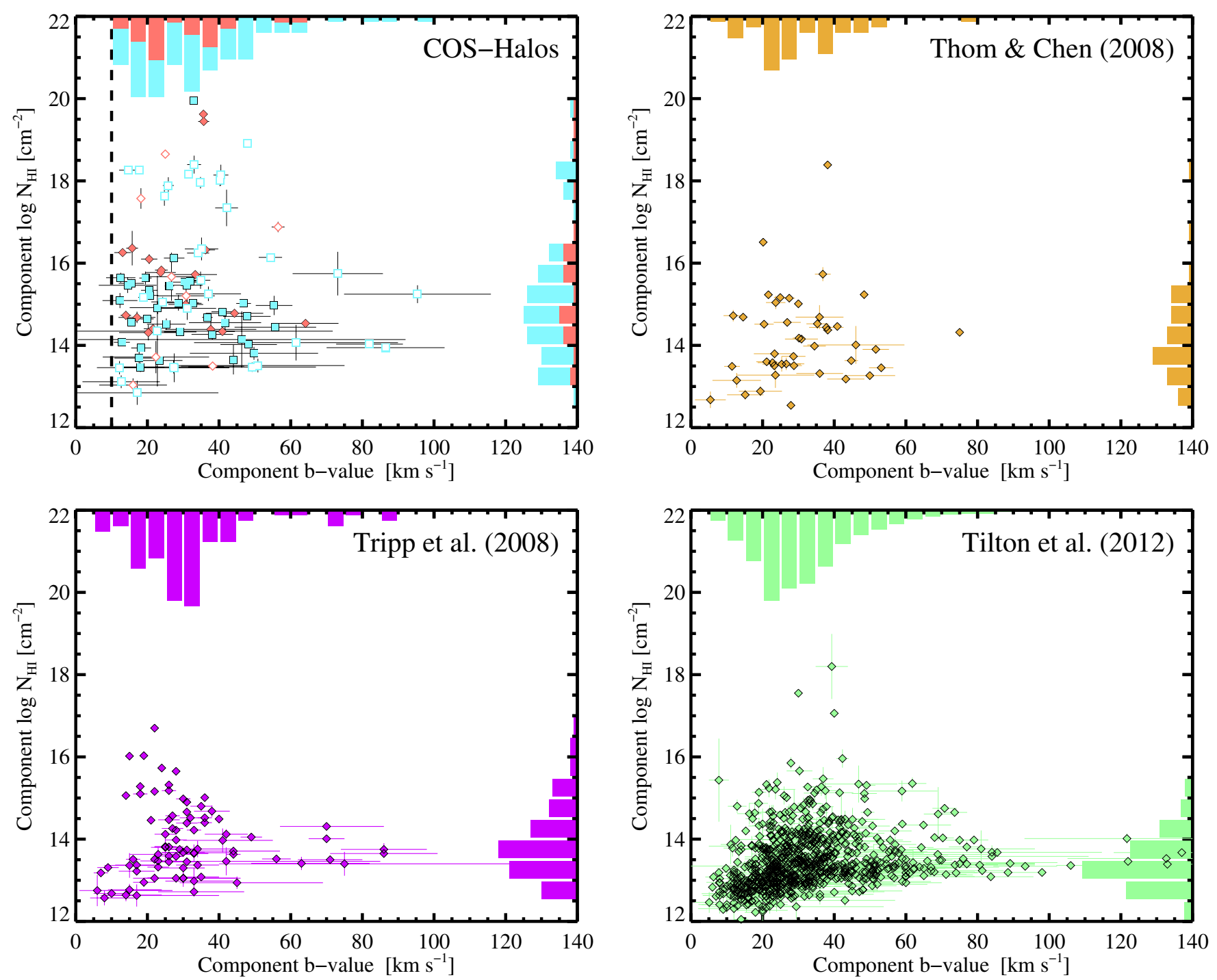

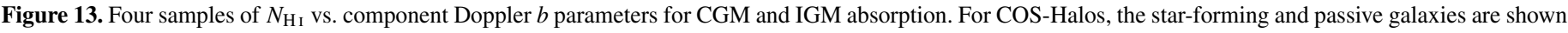

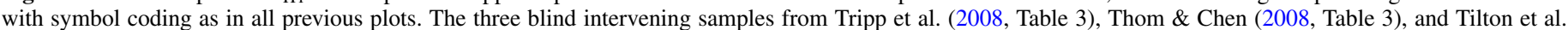

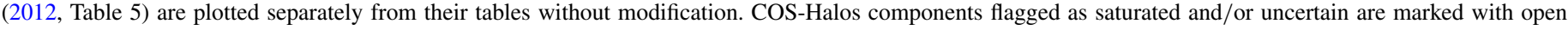
symbols. These column densities should be treated cautiously, though the given $b$-values can be considered reasonable upper limits to the true $b$.

(A color version of this figure is available in the online journal.)

nearby narrow components (e.g., Tripp et al. 2001; Richter et al. 2006), in many cases such absorbers could be hidden within the much stronger, narrower absorption that we detect, which is spread over $\sim 200-400 \mathrm{~km} \mathrm{~s}^{-1}$ and is often line-black saturated. Even BLA features with wings exposed at the edges of stronger, narrow absorption could easily go undetected at the typical S/N ratio of our data. This is clearly seen in the four $b \geqslant 80 \mathrm{~km} \mathrm{~s}^{-1}$ components in Table 5; the presence of these is required for formally good fits, but we regard their presence as truly BLAs as highly ambiguous - they could be continuum fluctuations, blended narrower components seen at low $\mathrm{S} / \mathrm{N}$, or other kinds of artifacts. We cannot confirm this with $\operatorname{Ly} \beta$, so we must regard these profiles as unconfirmed. They are, however, potentially good candidates for higher $\mathrm{S} / \mathrm{N}$ observations to determine their shape and kinematics. The widespread detection of a cold CGM does not preclude the hidden presence of a hot CGM traced by undetected BLAs. Gas at temperatures significantly above 1 million $\mathrm{K}$ would likely be undetectable in the UV under even the best circumstances.

\subsection{H I Properties by Galaxy Type}

COS-Halos was deliberately designed with both star-forming and passive galaxies in the sample to permit comparisons between these two populations of galaxies. The final sample has 16 passive or early-type galaxies and 28 star-forming galaxies. The chief goal of this design was to investigate an expected transition in the gas properties of galaxy halos around the stellar mass where they transition in star-forming properties, to see if halo gas plays some role in the quenching or continued suppression of star formation.

On the basis of the COS-Halos measurements analyzed so far, the evidence for a change in the behavior of gas halos between star-forming and passive galaxies is mixed. Tumlinson et al. (2011a) reported the bimodality of highly ionized gas traced by $\mathrm{O}$ vI, with a lower rate of detection (30\% versus $90 \%$ ) and weaker absorption in the COS-Halos ETG sample. However, as reported by Thom et al. (2012), there is much less difference in the presence or strength of cool gas traced 
by $\mathrm{H}_{\mathrm{I}}$ in the star-forming and passive samples. There are also modest differences in the covering fraction of low-ionization gas between the star-forming and passive subsamples Werk et al. (2013). Our sample of ETGs, combined with our measures of galaxy/absorber and internal gas kinematics, clearly indicates that gas with temperatures well below the halo virial temperature is common in the vicinity of passive galaxies (Thom et al. 2012) with a $75 \%$ rate of detection even down to low column density limits. This is only a weak rejection of the null hypothesis that the two galaxy subsamples exhibit a similar equivalent width distribution. Considering detections only, there is no evidence that the CGM H I around passive galaxies is significantly weaker or stronger than for star-forming galaxies. These findings led Thom et al. (2012) to conclude that the CGM of ETGs could harbor a cool, photoionized mass of $\sim 10^{10} M_{\odot}$ or more.

Figures 7-11 reinforce these results with detailed comparisons between the line kinematics and internal component structure of absorption in the two subsamples. Figure 10 shows that the kinematics of the $\mathrm{H}_{\mathrm{I}}$ with respect to the galaxy systemic velocity are not significantly different (a two sided K-S test rejects the null hypothesis at only $10 \%$ confidence), indicating that both sets of absorbers could be effectively bound to their galaxies. Figure 11 shows similar distributions of internal kinematics from linewidths and a lack of evolution with impact parameter. Thus the strong, cold, and bound medium traced by $\mathrm{H}$ i does not vary significantly from star-forming to passive galaxies, except in those $25 \%$ of cases where the latter do not show it.

In light of the COS-Halos results on H I strength, kinematics, and comparisons to galaxy properties, we conclude that a possibly bound, cool CGM is a generic feature of $L \sim L^{*}$ galaxies independent of their type. We have only statistically modest suggestions that passive galaxies exhibit $\mathrm{H}$ I less often than their star-forming counterparts. A larger sample of early type galaxies could address this suggestion with better statistics. We have robust evidence that the detected CGM has temperatures of $T \lesssim 100,000 \mathrm{~K}$, well below the expected virial temperatures of the halos that host these absorbers, but we cannot rule out the presence of some hot material that would go undetected. Finally, the relative velocities indicate that the detected gas is consistent with being bound to the host galaxy. None of these results shows a strong dependence on galaxy type, so we conclude that a cold, bound CGM is a generic feature of galaxies near $L^{*}$.

\section{THE STATE AND ORIGINS OF CIRCUMGALACTIC H I}

The COS-Halos measurements of $\mathrm{H}$ I are intended to characterize empirically the distribution and content of gas surrounding $L^{*}$ galaxies. In this section, we use our results and the previous studies to address some open questions about the origins of this gas. Is it really a CGM? What is its source-the CGM, or the surrounding LSS? How does it relate to satellites, disks, accretion, or feedback? What is its fate-has it recently escaped its host galaxy and hence is flowing out, or is it accreting to fuel star formation?

We examine two basic classes of explanation for the detected Hi. The basic division between the two classes depends on whether the gas is outside the galaxy's halo, or "inside" the galaxy's halo. For the purposes of this discussion, we define "inside" the halo to be inside the virial radius $R_{\mathrm{vir}}$.

First, in Section 6.2 we consider the class of explanation in which the gas resides in the LSS of the "cosmic web," and not directly in the galactic halos that we have targeted at $R \leqslant R_{\mathrm{vir}}$. Possible origins outside include IGM "filament" gas near the galaxy but outside the halo (Section 6.2.1), gas residing in a group environment of which the target galaxy is a member (Section 6.2.2), and finally gas arising from the halos of other galaxies nearby that are not the target, including gas driven out in winds or gas residing in nearby "minihalos," or small halos in the same LSS filament as the target (Section 6.2.3).

Then in Section 6.3 we consider the class of origins that lie within $R_{\text {vir }}$, including three basic origins: gas arising in galaxy disks themselves (Section 6.3.1), gas bound to or stripped from satellites (Section 6.3.2), and gas distributed through a diffuse medium in a true "CGM" of clouds and diffuse gas (Section 6.3.3). In the first case, the gas can arise directly in the galaxy disk ISM, or in ionized disks extending beyond the stars. In the second case, gas related to satellites can arise directly in their ISM, in DM bound, starless "minihalos," in gas ejected from satellites by their own star formation, or in material stripped by tidal forces or ram pressure from an ambient medium surrounding the host.

"True" CGM gas can arise in the small neutral fraction of an otherwise hot halo (near the virial temperature), denser clouds cooled from and pressure-confined by this hot medium (Mo \& Miralda-Escude 1996; Maller \& Bullock 2004), gas accreting in a "cold mode" below $T_{\text {vir }}$, gas accreting from the IGM in a "hot mode" (i.e., cooling flows), supernova winds or galactic fountain gas ejected from the host galaxy, or interface regions between cold clouds from one of these other sources and the expected hot corona.

Our goal in investigating these possible origins is not to conclusively rule out or confirm a particular unique explanation, as it is generally true that contributions from multiple sources are compatible with the data as well as any single scenario. Rather, our goal is to identify the properties that a particular model origin would have if adopted alone, and thereby to assess the relative viability of the various scenarios and their relative possible contributions. The purpose of these simple models is to aid our own interpretations of the data, and those of others, and possibly to guide follow-up observations. These simple models are best understood on that limited basis, not as unique or conclusive explanations of the empirical results, which speak for themselves.

\subsection{A Simple Model}

In the sections to follow, we will consider whether the detected H I can arise outside the virial radius $R_{\text {vir }}$ (either from the IGM or from other galaxies), inside $R_{\text {vir }}$ but not in a diffuse CGM (from satellites or extended galaxy disks), or whether it is most likely a true diffuse CGM. To assist with these tests, we have built a toy model of gas halos with very simple parameterizations that describe the geometry and ionization state of the gas. This model will be used below to assess the viability of the various origin hypotheses, so we describe its details here.

This "simple halo" model includes a spherically symmetric density profile running with physical radius $R$ specified by a power-law of particle density,

$$
n_{\mathrm{H}}=n_{0} R^{\alpha},
$$

where $\alpha$ is the power law slope and the density is normalized to cosmic overdensity

$$
\delta_{0}=n_{0} / \bar{n}
$$

at the given $R_{\text {vir. }}$. We take

$$
\bar{n}=\left(3 H^{2} / 8 \pi G\right) \Omega_{b} / m_{\mathrm{H}} \times(1+z)^{3}=4.3 \times 10^{-7} \mathrm{~cm}^{-3}
$$


at $z=0.2$. At each $R$, we calculate the ionization fraction of $\mathrm{H} \mathrm{I}$ given the local density, a fixed parametric temperature $T$, and the extragalactic ionizing background computed at $z=0.2$ (Haardt $\&$ Madau 2001). The ionization model assumes that the gas is optically thin and is in thermal and ionization equilibrium, the latter set by recombinations in balance with ionizations from photons and collisions with electrons and protons in collisional ionization equilibrium (CIE) at the parametric temperature. The ionization tables are given in terms of local density and temperature and return the ionization fraction for a range of particular observable ions; here we focus on the $\mathrm{H}$.

The spherical halo can be surrounded by either a medium with a density that continues to decline in density as $R^{\alpha}$, or by a fixed medium of constant parametric density $\delta_{\text {back }}$ over $R_{\text {vir }} \leqslant R \leqslant 1 \mathrm{Mpc}$. This external medium can represent a larger structure, such as a filament of LSS or intragroup medium, in which the model halo is embedded.

Once the density profile and ionization structure of the model halo are calculated, column densities are integrated along mock lines of sight through the medium at a range of impact parameters to compare to the COS-Halos data. These column densities are calculated by line-integrals along chords running through the spherical density profile. Finally, total masses of ionized gas are calculated for various regions in physical radius or in projection.

Naturally these simple models leave out important physical details such as density fluctuations, non-equilibrium ionization, and velocity fields among other potentially relevant effects. However, they are not intended to faithfully represent all the rich detail of the gas giving rise to the detected absorption. These models are useful for testing whether simple configurations and assumptions can explain the data or not, and to help derive simple estimates of the properties of the detected gas under certain assumptions. Their results should be considered in light of these significant limitations and modest goals.

\subsection{Gas outside $R_{\text {vir }}$ \\ 6.2.1. IGM Filaments}

First we examine the possibility that the detected gas lies outside $R_{\text {vir }}$ in extended filaments of gas associated with LSS in which the COS-Halos galaxies are embedded Figure 14 shows the results of this simple model for this case. The plot assumes $\alpha=-2$ and $T=30,000 \mathrm{~K}$. In addition to the COS-Halos data plotted as in all figures above, the black points show the mean and rms column densities from Prochaska et al. (2011b) as a characterization of $\mathrm{H}$ I surrounding $\sim L^{*}$ galaxies at $\gtrsim 300 \mathrm{kpc}$. In the upper panel, the blue curves show $N_{\mathrm{H}_{\mathrm{I}}}$ versus impact parameter for the full model, with the $\alpha=-2$ density profile normalized to $\delta_{0}=5-30$ at $R_{\text {vir }}=350 \mathrm{kpc}$. In this model, the steeply declining density profile adopted inside $R_{\mathrm{vir}}$ is extended (arbitrarily) out to $1 \mathrm{Mpc}$ to represent a filament of decreasing density. Here, the "observed" column density $N_{\mathrm{H}}$ does not decline as rapidly as $n_{\mathrm{H}}$, because the column density is obtained by a line integral over large pathlengths that compensate for the declining density. The range of adopted normalizations $\delta=5-30$ is reasonable for gas near the virial radius. The thick black curve marks a full model with $\delta_{0}=20$; the thin black curve truncates this model at $350 \mathrm{kpc}$, showing that the column densities outside $300 \mathrm{kpc}$ are produced predominantly by the "filament" and those inside predominantly by the "halo." The total masses in the spherical structure out to $1 \mathrm{Mpc}$ are $0.6-4 \times 10^{11} M_{\odot}$. This model adequately reproduces the data
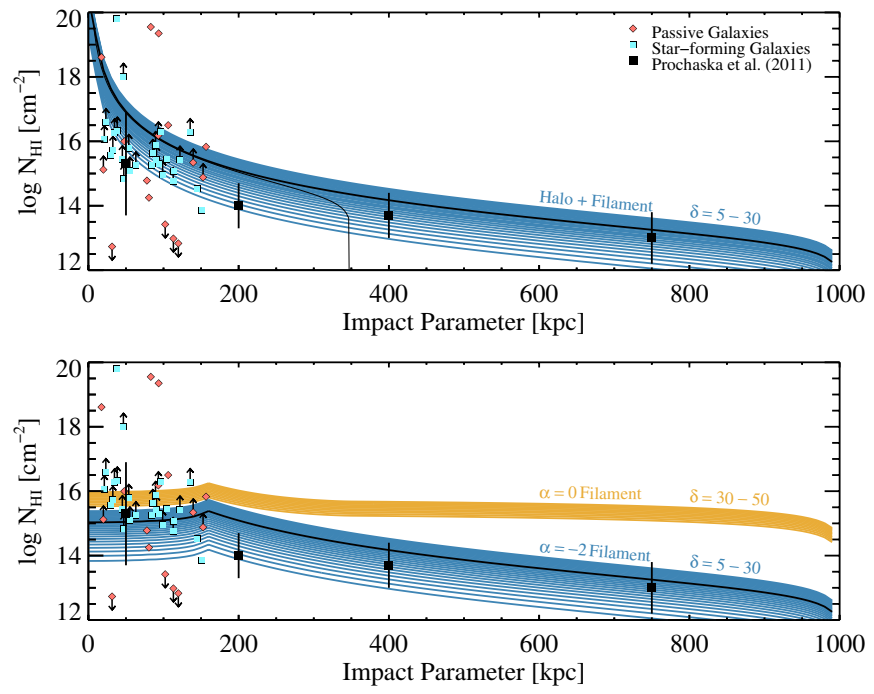

Figure 14. Simple models of halo and IGM gas compared to H I data. The COSHalos data are plotted as before. The black squares mark the median column density and rms scatter for all galaxies in Prochaska et al. (2011b). In the upper panel, the blue colored region marks the model density profile with $\alpha=-2$ described in the text normalized to overdensity $\delta=5$ to 30 at $R_{\mathrm{vir}}$ with constant temperature $T=30,000 \mathrm{~K}$. The black lines mark the curve with $\delta=20$, and then repeat it excising all gas at $>R_{\mathrm{vir}}$ (thin black curve). The simple model which extends gas to $1 \mathrm{Mpc}$ with declining density (e.g., the thick black curve) provides a reasonable description of the trend with impact parameter. In the lower panel, we repeat the same $\alpha=-2$ models with the spherical region inside $150 \mathrm{kpc}$ entirely removed. The orange curves increase the range of $\delta=30-50$ with the halo and again omit $<150 \mathrm{kpc}$. This model, which matches the COSHalos detections by including a diffuse, extended background outside $150 \mathrm{kpc}$, overproduces absorption far away from galaxies. The best fitting models are those in which gas density increases inside $R_{\text {vir }}$.

(A color version of this figure is available in the online journal.)

over a broad range of impact parameter by extending gaseous structures to high density at low radius (the "CGM") and low density at high radius (the "filament").

The lower panel of Figure 14 shows the behavior of models containing only this simple model filament, in two forms. The blue curves repeat the "halo+filament" $\left(\alpha=-2, \delta_{0}=5-30\right)$ models from the upper panel, but this time excises all the gas inside the $150 \mathrm{kpc}$ sphere covered by our data prior to the line integral to obtain $N_{\mathrm{HI}}$. This model expresses the (extreme) scenario in which all the gas "observed" at $<150 \mathrm{kpc}$ impact parameter actually sits at $>150 \mathrm{kpc}$ radius from the center of the system. These "halo deleted" models clearly fall short of the COS-Halos detections at $\leqslant 150 \mathrm{kpc}$, though they still provide a reasonable match to the data points at larger impact parameters. By deleting the gas from the region surveyed by COS-Halos, they fail to reproduce the trend to higher $N_{\mathrm{HI}}$ observed in the two samples of data.

To assess whether a more extreme "filament" model may be able to restore the fit when gas inside $150 \mathrm{kpc}$ is deleted, the solid orange curves in the lower panel assume that the density $\delta_{0}$ at $R_{\text {vir }}$ is continued at a constant value out to $1 \mathrm{Mpc}$; this is a "constant-density" filament. ${ }^{22}$ Once again gas is deleted inside $150 \mathrm{kpc}$. To better match the data in the COS-Halos region with this "constant-density" filament, we must increase the range $\delta_{0}=5-30$, as in the lighter curves, to $\delta_{0}=30-50$. This model, which matches the COS-Halos detections by including a diffuse, very extended background but omitting the halo, overproduces 22 The "filament" must have $\delta_{0}$ less than or equal to the halo at the virial
radius, by the definition of virtualized halos. 
absorption far away from galaxies (the implied mass within $1 \mathrm{Mpc}$ is $\left.1-2 \times 10^{12} M_{\odot}\right)$. Thus, "filament" gas can help explain the data at $>R_{\text {vir }}$ but fits best if the density continues to decline outside the halo (top panel). More extreme models which attempt to explain the data by either (1) deleting gas at $<150 \mathrm{kpc}$ from a filament with a declining density profile (top), or (2) including only a constant-density filament at $>150 \mathrm{kpc}$ (bottom) both fail to recover the trend of $\mathrm{H}$ I strength seen in the data. This examination of these simple models leads us to a simple explanation: that the detected $\mathrm{H}$ i is not likely to be caused simply by LSS filament gas, with no contribution from within the halo itself. It remains possible that some smaller portion of the detected material arises from such LSSs, but this modeling indicates that the contribution is of order $\log N_{\mathrm{H}} \sim 14-15$ or less. More detailed examinations of these models and the behavior of $\mathrm{H}$ I gas within $1 \mathrm{Mpc}$ of galaxies is deferred for a later study.

\subsubsection{Intra-group Gas}

It is also possible that gas observed at low impact parameters near galaxies arises outside $R_{\text {vir }}$ but within a galaxy group. Such "intragroup" gas has been claimed in some circumstances where groups are evident and gas alignment with any particular galaxy is not obvious (e.g., Shull et al. 2003; Aracil et al. 2006). We do not have the data necessary to perform a fair test of this hypothesis with COS-Halos, for two reasons (see also Section 2.5). First, our original selection of candidates actively selected against galaxies with coincident photometric redshifts, because the goal was to obtain a sample of isolated $L^{*}$ galaxies to minimize confusion. Nevertheless some galaxies turned out to have neighbors at the same redshift owing to imperfect selection and photometric redshift errors. Second, we have not obtained redshifts for all galaxies in these fields, and so we cannot make robust statements about neighbors and possible group membership in all cases.

Despite this original selection against groups and the heterogeneous redshift database, we do know of a few targets galaxies that are likely in groups, defined as those with one or more nearby galaxies at the same redshift with similar luminosity. Six of our galaxies meet these criteria (Werk et al. 2012), and their detections in $\mathrm{H}_{\mathrm{I}}$ show a wide degree of diversity. Two exhibit subDLA systems (J0925+4004 galaxy 196_22 and J0928+6025 galaxy 110_35), three show strong H I with multiple components (J0910+1014 galaxy 242_34, J0820+2334 galaxy 260_17, J1133+0327 galaxy 110_5), and one is a non-detection H I (J2257+1340 galaxy 270_40). This diversity means that we cannot make firm statements about group gas origins in those COS-Halos galaxies for which group membership is known. The nearest-neighbor analysis indicates that their large-scale environments $(1-5 \mathrm{Mpc})$ are not unusual for galaxies at this luminosity (Werk et al. 2012).

However, despite the lack of detailed knowledge of group membership for the COS-Halos galaxies, we regard it as unlikely that group gas is a dominant cause of the detected absorption in $\mathrm{HI}_{\mathrm{I}}$ because of the challenging set of observational facts that a group model must explain. First, the gas we detect is apparently within $R_{\mathrm{vir}}$ for the selected galaxies. Second, the relative velocities are well within the expected escape velocities and tend to be centered on the systemic redshift of the targeted galaxies (Section 4.2 and Figure 10); even in poor groups the velocity dispersions of a few hundred $\mathrm{km} \mathrm{s}^{-1}$ would not be expected to show such a trend. Third, we find a nearly unity covering fraction of gas near $L^{*}$ galaxies; this would imply a nearly unity covering fraction in an intra-group medium if it were not associated with particular galaxies. Fourth, there are relatively clean trends of $\mathrm{H}$ I strength with impact parameter, which would not necessarily be expected if the gas reside in an intragroup medium but is not associated with particular galaxies.

To prove that this gas actually arises in an intra-group medium instead of inside the virial radius, the statistical associations of the gas properties with group membership must be as strong as or stronger than correlations with the nearest galaxy individually. Evidence for this would include findings that group membership causes a systematic change in gas properties-stronger, weaker, more extended, hotter, etc. This would require a sample of galaxies within groups and a control sample of galaxies that are not in groups. It must also be shown that the apparent change in absorption properties in a group sample is not caused simply by the superposition of gas inside the $R_{\text {vir }}$ of group members, as has been claimed for, e.g., Mg II-traced CGM gas by Bordoloi et al. (2011). No such controlled experiment has yet been done for $\mathrm{H}$ I and other UV-band ions. Should such a sample of selected group sightlines exist, a subset of COS-Halos fields containing galaxies outside groups could serve as a control sample once their spectroscopic followup is complete.

\subsubsection{Other Nearby Galaxies}

Apart from galaxies nearby our targets that share the same group-scale DM halo, it is possible that some of the detected gas is associated instead with interloper $L^{*}$ galaxies nearby that create chance projections along the sightline (less luminous satellites are considered separately below). This material could be halo gas within, ejected from, or bound to the satellites of the neighbor. Interloping galaxies could lie within a few Mpc in the foreground or background of our sample galaxies, far enough for significant Hubble flow velocities with respect to our targets, and still have peculiar velocities move them back into chance coincidences in velocity space.

Chance coincidences such as this are extremely difficult to rule out conclusively, particularly for individual cases, but are disfavored by our selection technique and by our knowledge of the fields. Even though we have not completely surveyed all these galaxy fields to identify all possible interlopers, the comments above regarding possible group membership cover all the cases where neighboring $L^{*}$ galaxies at the same redshift were identified. No other targeted systems have massive galaxies closer to the sightline than the target and at similar redshift, as a consequence of active selection for isolated $L^{*}$ galaxies. Any possible interlopers would need to have $L \ll L^{*}$ or impact parameter $\gg R_{\text {vir }}$, thus begging the question of how $\mathrm{HI}_{\mathrm{I}}$ is distributed around galaxies. Also, any interlopers would not be distributed almost evenly in impact parameter to the sightline as the targeted galaxies are (by selection). The absorption they contributed would then be drawn preferentially from larger radii and would not be expected to produce the clean trends with target impact parameter that are shown in Figures 7, 12, and 14. That is, absorbers drawn from well-behaved relations around randomly distributed galaxies are not expected to recover well-behaved relations around these carefully selected galaxies. The contribution of interlopers in velocity space should be just as likely to lie outside the escape velocity of the targeted galaxy as within it, not to give the tight kinematic correlations seen in Figure 10. Given the suppression of interlopers in our selection and subsequent redshift screening, and these qualitative considerations about the observed trends, we regard it as unlikely that neighboring $L^{*}$ galaxies contribute significantly 

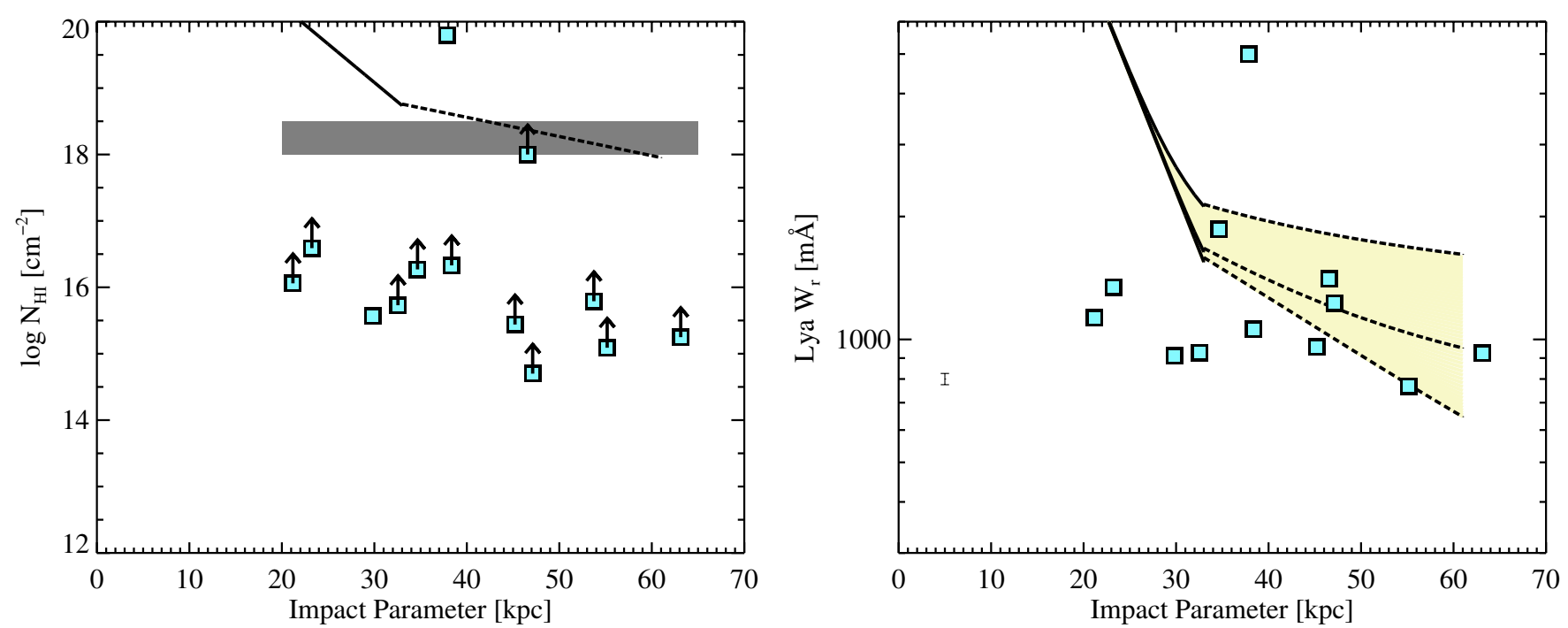

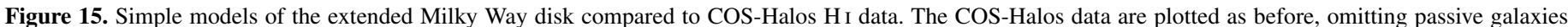

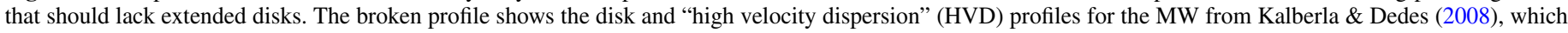

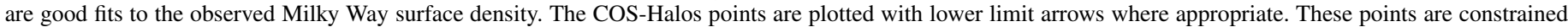

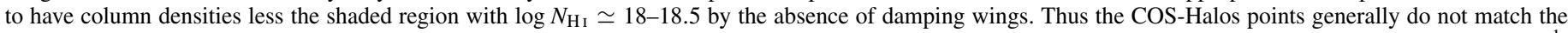

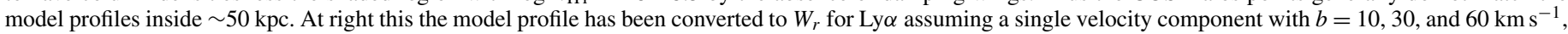

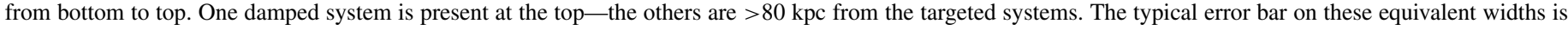
smaller than the adopted symbol size, as shown by the representative value at left in the panel.

(A color version of this figure is available in the online journal.)

to the detected absorption around our targeted galaxies. Finally, we note that attempting to construct a quantitative model for the contribution of interloper $L^{*}$ galaxies (as we will do for satellites below) would beg the question of how diffuse $\mathrm{H}_{\mathrm{I}}$ is distributed in space and velocity around $L^{*}$ galaxies. A full treatment of this issue would require deeper, complete galaxy surveys in these fields.

\subsection{Gas inside $R_{\text {vir }}$}

\subsubsection{Extended Galactic Disk Gas}

An obvious possible explanation for $\mathrm{H}_{\mathrm{I}}$ inside $R_{\mathrm{vir}}$ is gas arising in the ISM of the galaxy itself. This can be either the familiar, predominantly neutral inner disk or extended disks kept ionized by internal or external sources. Using $21 \mathrm{~cm} \mathrm{H}$ I surveys of the MW as a template for star-forming disk galaxies (Kalberla \& Kerp 2009), we expect that gas-rich disks themselves should imprint a DLA $\left(\log N_{\mathrm{H}_{\mathrm{I}}} \geqslant 20.3\right)$ or at least a strong LLS $\left(\log N_{\mathrm{H}_{\mathrm{I}}} \gtrsim 18\right)$, but only within $\lesssim 35 \mathrm{kpc}$. COS-Halos has 4 star-forming galaxies at $<35 \mathrm{kpc}$ and 12 at $<60 \mathrm{kpc}$ impact parameters. We do not expect strong $\mathrm{H}$ a absorption from gaseous disks in our passive subsample.

In making these comparisons, we remain in the spirit of simple models from above and so we adopt the fitted MW surface density profile from Kalberla \& Dedes (2008) as a fiducial gas disk. This profile follows an exponential profile out to $35 \mathrm{kpc}$, $\Sigma_{\mathrm{HI}}=30 \exp \left(\left(R-R_{\odot}\right) / R_{S}\right) \quad M_{\odot} \mathrm{pc}^{-2}$, with $R_{S}=3.75 \mathrm{kpc}$. Outside $\sim 35 \mathrm{kpc}$, the profile flattens to a shallower dependence on galactocentric radius. Kalberla \& Dedes (2008) treat this as an extension of the main disk, but one which may consist of numerous small clouds or a turbulent medium with high velocity dispersion. This part of the profile assumes a model instead of being a direct fit to the emission data. Figure 15 shows the two components of the MW profile compared to COS-Halos data inside $70 \mathrm{kpc}$. The main hindrance to direct comparison is the substantial fraction of saturated systems with
$N_{\mathrm{H} \text { I }}$ lower limits. Therefore, we perform these comparisons as before in both $N_{\mathrm{H} \text { I }}$ and $\mathrm{Ly} \alpha$ equivalent width. We note that the saturation effects manifested as lower limits in Figure 15 are no longer an issue above $\log N_{\mathrm{HI}} \simeq 18-18.5$ (gray box), where damping wings usually appear; their absence from the observed profile can loosely constrain $N_{\mathrm{H}_{\mathrm{I}}}$ to $\lesssim 18.5$, above which robust measurements can be derived from fitting the damping wings.

It is notable that the strong damped or sub-damped absorbers $\left(\log N_{\mathrm{HI}} \gtrsim 19\right)$ that would be expected inside $\sim 30 \mathrm{kpc}$ are not evident in both panels of Figure 15. Because of their geometry, disks can be missed in cases where the target galaxy appears near edge-on with respect to the QSO sightline. We do not know the disk sizes or inclinations of our star-forming subsample, so any contribution of disks to COS-Halos would be that of typical gas disks in $\sim L^{*}$ galaxies that are randomly oriented and inclined with respect to the line of sight. In such a sample, we would expect that the disk absorption would still cover approximately half of the area out to some impact parameter on the sky if it fills the disk out to that same radius in the galaxy. Thus the absence of any clearly damped, disk-line absorption inside $\sim 30 \mathrm{kpc}$ is somewhat puzzling, though with small numbers it may still be attributable to the accidents of random viewing geometry or to the fact that the typical stellar mass for COS-Halos galaxies is slightly smaller than the MW.

The outer portion of the MW surface density also seems to over-predict the absorption seen in COS-Halos. This effect is best seen in the right panel of Figure 15, where we have converted the MW column density versus $R$ into an equivalent width for single-component absorption using a curve of growth with $b=10,30$, and $60 \mathrm{~km} \mathrm{~s}^{-1}$ from bottom to top. The two larger velocity dispersions are more characteristic of the fitted components in COS-Halos (see Figure 11). Even these profiles exceed the data points from COS-Halos, suggesting that the MW profile at $R>30 \mathrm{kpc}$, if is an extended disk, does not match up with external galaxies. However, this disagreement does not necessarily imply that COS-Halos does not detect 
ionized, extended disks in some cases. Observed H I disks can have quite sharp edges induced by photoionization from an external ionizing background (Maloney 1993; Dove \& Shull 1994), with the location of the edge depending on where the total density profile effectively becomes optically thin. Also, an extended photoionized disk could be difficult to distinguish from a more diffuse halo medium in general; the latter might be expected to continue the galaxy's rotation curve while the former might not. Since we lack measurements of the galaxy inclination and orientation with respect to the line of sight, and also any information about their rotation curves, we cannot yet perform the relevant tests. We, therefore, conclude that COSHalos likely probes the region of space where the disk transitions to general halo gas, but we cannot cleanly separate them with the present dataset. We leave a more sophisticated analysis of these ideas to future work.

\subsubsection{Gas in and from Satellite Galaxies}

It is possible that some fraction of the detected gas is bound to, or has been recently stripped from, satellite galaxies surrounding the targeted $L^{*}$ galaxies whether bound to them or not. We would like to assess how much of a contribution satellite galaxies can make to the observed column density and absorption profiles as a population. It is straightforward to assess the possible contribution of gas bound to satellites within the well-specified structure-formation model with simple assumptions about gas inside the satellites. It is more difficult to assess the contributions of gas stripped from satellites. We will do the former first, and see if that gives any insights into the budgets of stripped gas.

First, let us recall the observational information to be explained: We detect $N_{\mathrm{HI}} \gtrsim 10^{14} \mathrm{~cm}^{-2}$ with nearly unity covering fraction at all impact parameters $<150 \mathrm{kpc}$, for both galaxy types. The covering fractions at $>10^{15} \mathrm{~cm}^{-2}$ are $\gtrsim 0.5$. The kinematic spread of the absorption is equally important as a constraint. The detected absorption is usually distributed into a few resolved components that appear to be $b \sim 20-40 \mathrm{~km} \mathrm{~s}^{-1}$ (Figure 11). The range of centroid velocities for the identifiable components is roughly $\pm 100 \mathrm{~km} \mathrm{~s}^{-1}$ (see Figure 10). The absorption beyond that out to the typical edges of the full profiles near $\pm 200 \mathrm{~km} \mathrm{~s}^{-1}$ is partially caused by broadening of individual components (e.g., curve-of-growth effects, whether thermal or non-thermal). Given the $20 \mathrm{~km} \mathrm{~s}^{-1}$ resolution of COS, there is a strong possibility of narrow unresolved components inside the saturated profiles and the distinct possibility that what appear as single $b \sim 20-40 \mathrm{~km} \mathrm{~s}^{-1}$ components are actually composed of narrower blended components (indeed this is often indicated by component structure in metal ions observed at higher resolution, e.g., Mg II; Werk et al. 2013). However, any model must still match the total kinematic extent of the detected absorption, which we take to be $\pm 100 \mathrm{~km} \mathrm{~s}^{-1}$ from the typical range of component centroids. The high covering fractions and broad kinematic extent of the detected $\mathrm{H}$ I jointly provide robust data that any model involving satellites must match.

We regard the three high-column density sub-DLA H I systems $\left(\log N_{\mathrm{HI}}=19.4-19.9\right)$ as the most likely of all the COS-Halos systems to arise in the bound ISM of satellites galaxies, though extended H I disks and high-column "HVC-like" origins are also possible. These systems were previously analyzed by Meiring et al. (2011) and Battisti et al. (2012), who found them to have modestly sub-solar to super-solar metallicities, $[\mathrm{Z} / \mathrm{H}] \sim-0.4$ to +0.3 . The lower of these metallicities are consistent with luminous dwarf satellites, while the higher metallicities may indicate an extended galactic disk (but see above).
In all three cases, we have not confirmed any galaxy redshifts closer to the sightline than the targeted $L^{*}$ galaxies.

In the MW system, $\sim 17 \%$ of the sky is covered by $21-\mathrm{cm}$ HVC gas at $\log N_{\mathrm{HI}} \gtrsim 18.5$ (Wakker 1991) and $37 \%$ is covered at $\log N_{\mathrm{HI}} \gtrsim 17.9$ (Murphy et al. 1995). The majority of this area is covered by the HVC cloud complexes, which are not known to be affiliated with particular satellites or stellar populations. The areal covering factor contributed by the prominent Magellanic Stream is only $\sim 5 \%$; and only $\lesssim 1 \%$ for the bound ISM of the Clouds themselves, even though their collective mass far exceed that of the HVC complexes. Thus if we take the MW system as a template (and ignoring the obvious differences in viewing geometry) we expect that $\lesssim 5 \%-10 \%$ of COS-Halos systems should show $\log N_{\mathrm{HI}} \gtrsim 19.5$, with most of the covering fraction arising in HVC-like gas clouds without stars and a still smaller fraction from gas bound to larger satellites. These expectations are borne out in Gauthier et al. (2010), who found that satellites of halos near the upper end of the COS-Halos range $\left(M_{\text {halo }} \sim 10^{13} M_{\odot}\right)$ should give a covering fraction of roughly $3 \%$ or less inside $150 \mathrm{kpc}$, using strong absorption ( $>1 \AA$ ) by $\mathrm{Mg}$ II as the proxy for strong absorption by cold gas. These results appear consistent with the model of Herenz et al. (2013), who translated the covering fractions of MW HVC lowions to an external viewing geometry and found that this HVC population would yield a Mg II covering fraction (for > $300 \mathrm{~m} \AA$ absorption) of $20 \%$ out to $60 \mathrm{kpc}$. Two of the three damped systems in COS-Halos have $W_{r}>1 \AA$ in Mg II (Werk et al. 2013). While we cannot definitely conclude that these damped systems arise in the ISM of satellites, their properties and hit rate are consistent with this explanation.

For the lower column density COS-Halos systems $\left(\log N_{\mathrm{HI}}<\right.$ 19), the possibility of satellites contributing significantly to the detected $\mathrm{H}$ I absorption exists but is more difficult to assess. The chief difficulty is our ignorance of the mass and extent of ionized gas (below the $21 \mathrm{~cm}$ detection threshold) surrounding satellites of varying mass. Building such a detailed model would have to assume a density profile of $\mathrm{H} \mathrm{I}$, around lower-luminosity satellites, where we have little if any empirical guidance. So, instead of building a detailed model of H I surrounding dwarf satellites, and then computing their covering fraction with the survey region, we attempt to work out the maximum possible cross section of gas bound to satellites from a simple but physically motivated picture of DM substructure. The maximum possible number of satellites which could contribute gas to the detected absorption is limited to the number of DM subhalos in host halos of $\log M_{\text {halo }}=11.5-13$. The number density of subhalos, each characterized by its maximum circular velocity $v_{\max }$, increases as $v_{\max }^{-3}$, so most of the available subhalos are "minihalos" that may or may not contain stars or ionized gas.

For concrete estimates, we use a subhalo catalog from the DM-only simulation of an $L^{*}$-like Via Lactea II halo by Diemand et al. (2007), which resolves subhalos down to $v_{\max }=4 \mathrm{~km} \mathrm{~s}^{-1}$. We make two additional assumptions. First, that subhalos cannot host gas with an internal velocity dispersion that exceeds their own: that is, they cannot contribute to the observed gas profiles a velocity width that significantly exceeds their own $v_{\max }$ (we assume that these velocities map directly to the Doppler $b$ parameter of fitted components). Second, that they cannot hold onto gas that falls outside their own instantaneous tidal radius $R_{\text {tidal }}$, as given by the VLII catalogs. We compute the cross section for absorption of all the VLII subhalos by assuming that they all have unity covering fraction of gas with $\log N_{\mathrm{HI}}>10^{15} \mathrm{~cm}^{-2}$ inside their own tidal radius. We include 


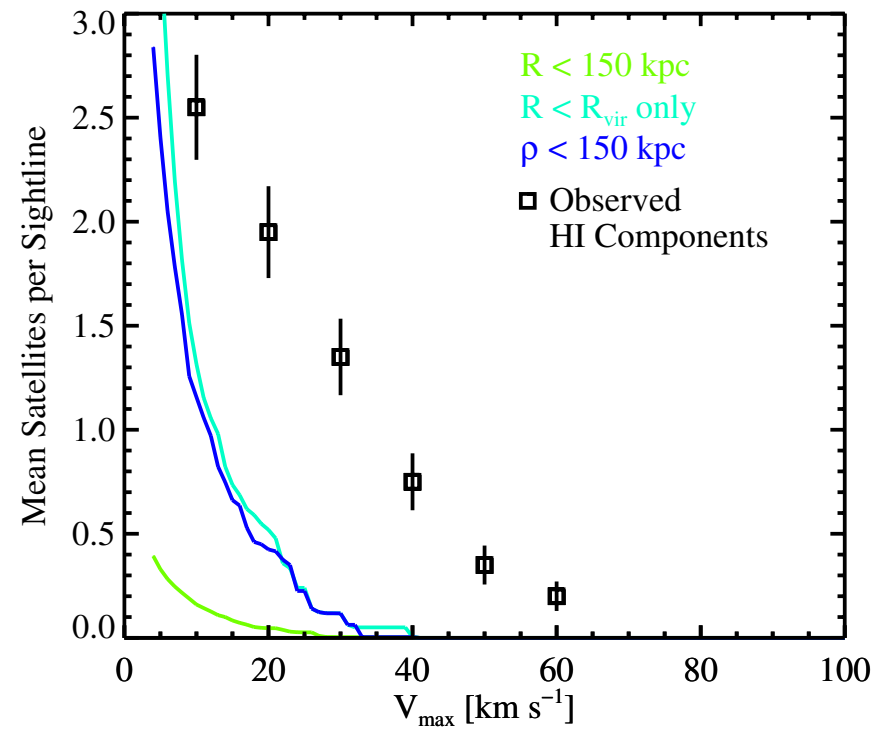

Figure 16. Mean number of satellites encountered by mock sightlines through the Via Lactea halo compared with the number of detected Hi components in the COS-Halos data. The solid curves show the cumulative (mean) number of satellites expected per sightline, integrated down to the $v_{\max }$ given on the $x$ axis. The model VLII subhalos with $\mathrm{H}_{\mathrm{I}}$ at unity covering fraction out to their tidal radius $R_{\text {tidal }}$. The mean number of satellites (or "covering fraction") can exceed unity because we allow for multiple components or satellites along single lines of sight. The data points use the profile-fitting results shown in Figure 10 to estimate the covering fraction of detected $\mathrm{H}$ I components as a function of Doppler $b$ parameter. We assume that the Doppler $b$ parameters map directly to $V_{\max }$ for satellites; this is merely an approximation. Even under these generous assumptions the subhalos fail to explain more than a minority of the detected HI. The three model curves show the VLII results for all subhalos within $150 \mathrm{kpc}$ physical radius of the host (light green), all subhalos within $R_{\mathrm{vir}}$ of the host (cyan) and, in blue, all subhalos that lie within a projected $150 \mathrm{kpc}$ impact parameter of the host, whatever their physical radius (as viewed from a randomly chosen orientation). The latter model occupies a cylindrical volume that best approximates the COS-Halos viewing geometry.

(A color version of this figure is available in the online journal.)

the small contribution of subhalos outside $R_{\mathrm{vir}}$ of the VLII host. The results of this very simple model are shown in Figure 16. Here, we also take at face value the distribution of profilefitting $b$-parameters in Figure 10 as an approximation to the distribution of detected components versus line broadening. The mean number of components per sightline is just above 2.5 at to the lowest limit of the reliable linewidths $\left(10 \mathrm{~km} \mathrm{~s}^{-1}\right)$.

If we assign detectable gas extending to $R_{\text {tidal }}$ for all VLII subhalos inside an "impact parameter" of $150 \mathrm{kpc}$ from the host, the mean number of satellites per sightlines (equivalent to the areal covering factor, but allowed to exceed unity) of subhalos down to $v_{\max }=20 \mathrm{~km} \mathrm{~s}^{-1}$ is 0.5 , but the mean number of components in the data is already well in excess of unity. This velocity is important as the value below which Local Group satellites do not contain detectable $\mathrm{HI}$, so using halos below this value in this model presumes that such halos can retain bound, ionized gas at the low levels detected in COSHalos. The VLII curve does not reach unity unless we use all subhalos down to $v_{\max }=10 \mathrm{~km} \mathrm{~s}^{-1}$, and it does not reach 2 until we include all subhalos down to $v_{\max }=6 \mathrm{~km} \mathrm{~s}^{-1}$. In other words, to match the COS-Halos data we must allow for unity covering fraction of $\mathrm{HI}$ in small subhalos that are not known to retain gas at all. Note that this problem only gets worse if we account for the finite resolution of COS and posit that the detected components may conceal narrower unresolved components. Narrower components can arise in subhalos of lower $v_{\max }$, but they are then more numerous in the data, forcing the points in Figure 16 to shift up while the model curves do not (e.g., there would typically be $>2$ per sightline if they are narrow and unresolved). Thus, under assumptions that maximize the cross sections of satellite DM halos (including minihalos), and conservatively estimating the number counts of detected gas components, subhalos fail to match the data by a large factor.

In short, we find that even if we generously assign all DM subhalos with H I gas at the detected level out to their tidal radii, their projected area and kinematic widths are not sufficient to explain the strong, broad absorption surrounding the COS-Halos galaxies. Of course, the satellite frequencies inferred from this exercise are not negligible (though the assumptions deliberately maximize them), so it remains possible that individual systems trace gas bound to satellites. It might be that a significant minority of the components and/or systems arise in gas bound to satellites, but if that fraction exceeds about one-half of all systems then our simple models imply that low-mass galaxies retain small portions of ionized gas that is undetected by $21 \mathrm{~cm}$ measurements. Proving or disproving this hypothesis in single cases would be very difficult if not impossible given only the $\mathrm{H}$ I data and lacking deeper images and spectroscopy of these fields. For now, we regard gas bound to satellites as CGM gas of interest that ultimately contributes to the mass budget of the host galaxy like any other CGM component. The origins of CGM gas in satellites is a possibly fruitful line of research that could be addressed in the COS-Halos data on metal lines, in new spectroscopic data on dwarf galaxies from our Cycle 18 HST program (PID 12248, "COS-Dwarfs"), and in the context of highly resolved numerical simulations of galaxy halos that could assess the relative contributions to the CGM of gas bound to satellites and gas arising in the diffuse CGM.

Gas stripping from satellites surely contributes to the CGM (viz., the Magellanic Stream) but the contributions of recently stripped material to the COS-Halos data is even more difficult to assess quantitatively than the possibility of gas bound to satellites. Stripping could increase the H I cross sections of small satellites, but to conserve mass it must then lower the typical column density. We do not have quantitative constraints on either cross-sections or column densities for real satellites at $\log N_{\mathrm{H}_{\mathrm{I}}}<10^{18} \mathrm{~cm}^{-2}$. Note that even if we were to crudely account for tidal stripping effects by arbitrarily assigning gas out to $2 R_{\text {tidal }}$ in the simple model above, the expected number of components would still not match the data without the contribution of small subhalos that are not known to contain gas. This is another area where insight from numerical simulations would be helpful.

\subsubsection{The CGM: Diffuse Gas within $R_{\text {vir }}$}

Having evaluated many sources of the detected $\mathrm{H}$ I absorption from outside the host halo, and from disks and satellites within it, we now turn to examining the properties that this medium has if it is a true "CGM": diffuse gas surrounding the galaxies that is not directly bound to satellites or arising in the IGM. This CGM might include flows on their way into the galaxy, ejecta on their way out, gas stripped long before from satellites by tidal forces or ram pressure, material that is being heated by active feedback or material cooling and falling in from the IGM. Perhaps the real CGM contains gas from all these sources.

COS-Halos has generated a rich dataset of multiphase ions that can be used to examine the ionization state, metallicity, kinematics, and origins of this gas using a range of diagnostic lines. An empirical analysis of the metal line survey is available 

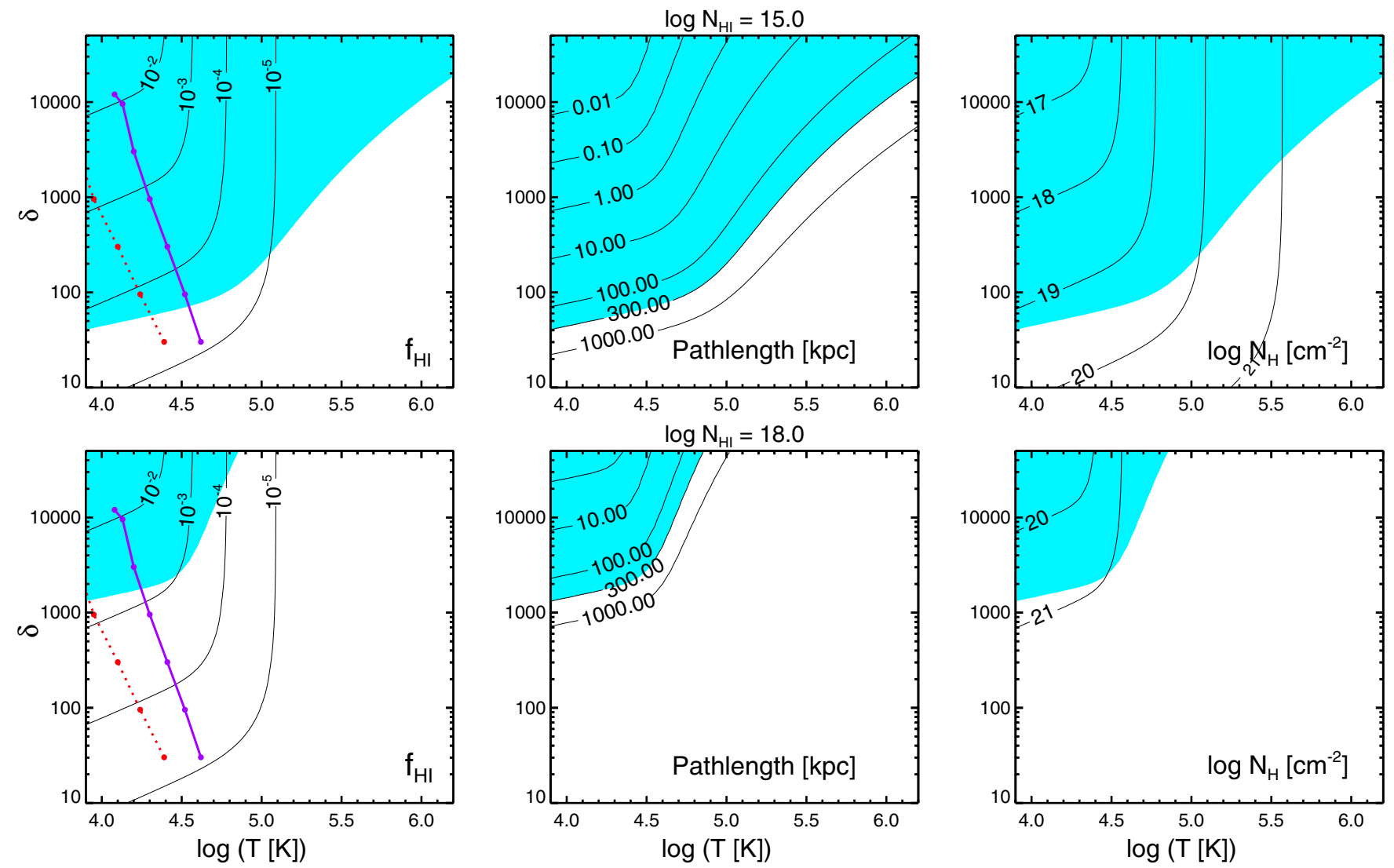

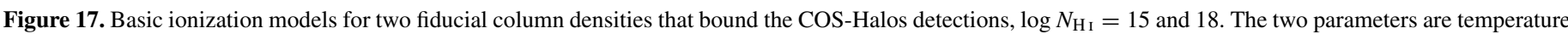

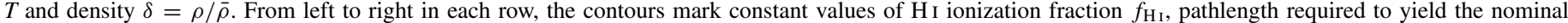

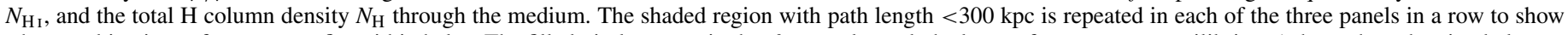

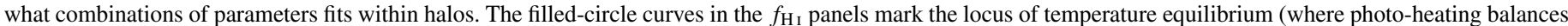
radiative cooling) for solar metallicity (dashed red) and 0.1 solar metallcity (solid purple).

(A color version of this figure is available in the online journal.)

in Werk et al. (2013). The H I by itself provides too little information on which to draw conclusions about the true state and origins of the CGM gas, but we can undertake simple models to get order-of-magnitude estimates for the properties of the CGM gas that might exist around these targeted halos. These models are rather limited in the amount of detail they can capture and still be constrained usefully by the H I data alone. Among the things we do not know are the density and temperature of the absorbing material, its distribution throughout the halo, the degree of clumping in space or along the line of sight, the thermal history of the gas, or its internal kinematics and bulk flows. In light of all these missing elements, we instead have only two modest aims: (1) to show that a diffuse ionized medium can reproduce the observed column densities and trends with galaxy properties and still fit within the spatial extent of halos, and (2) to estimate the properties-density, temperature and implied masses-for simple parameterizations of diffuse CGM that match the data.

The first kind of ionization modeling is designed simply to show that a diffuse ionized medium can reproduce the column densities we observe for plausible physical conditions and still fit within the physical extent of the relevant DM halos. To do this we model column densities from a uniform diffuse medium of constant temperature $T$ and constant density $\rho$, expressed in a ratio to the cosmic mean baryon density as $\delta_{0}=n / \bar{n}$, where $\bar{n}=4.2 \times 10^{-7} \mathrm{~cm}^{-3}$. This optically thin medium is exposed to the extragalactic ionizing background scaled to $z=0.2$ (Haardt
\& Madau 2001). In addition to photoionizations we also include temperature-dependent ionization from collisions in pure CIE. Thus we have a two-parameter space, as shown in Figure 17. The two rows show results for a fiducial column density $N_{\mathrm{HI}}=10^{15} \mathrm{~cm}^{-2}$ (top) and $N_{\mathrm{HI}}=10^{18} \mathrm{~cm}^{-2}$ (bottom); these two limits bound the region of our (non-damped) detections. The three columns are (left to right) contours of constant $\mathrm{H}$ I neutral fraction $f_{\mathrm{HI}}$, pathlength required to achieve the nominal $N_{\mathrm{HI}}$, and the total $N_{\mathrm{H}}$, for each possible combination of $\delta_{0}$ and $T$. The light blue shaded region shows where the implied pathlength is $300 \mathrm{kpc}$ or less, and is repeated in all panels to show allowable values of the parameters by this criterion.

The range of plausible temperatures in this space is further constrained by the line-broadening observed in COS-Halos systems. The fitted line widths constrain most of the detected gas to $T \lesssim 10^{5} \mathrm{~K}\left(b \leqslant 40 \mathrm{~km} \mathrm{~s}^{-1}\right)$, or roughly $f_{\mathrm{H}_{\mathrm{I}}} \gtrsim 10^{-5}$. Basic considerations of temperature equilibrium (red and purple curves in Figure 17) and cooling also argue for temperatures of $10^{4-5} \mathrm{~K}$. Cooling and photo-heating timescales also argue for temperatures to be $10^{4-5} \mathrm{~K}$, near the locus of points indicating thermal equilibrium (red and purple curves in Figure 17) At $T=10^{5} \mathrm{~K}$ and $\delta=1000$, the cooling time is only $20 \mathrm{Myr}$ at metallicity $Z_{\odot}$ and $65 \mathrm{Myr}$ at $0.1 Z_{\odot}$, and scale down inversely with $\delta$. On the equilibrium locus, radiative cooling balances photo-heating exactly and the cooling time is effectively infinite. Gas that takes excursions away from the equilibrium curves will not remain there for long. Gas with the observed kinematics 


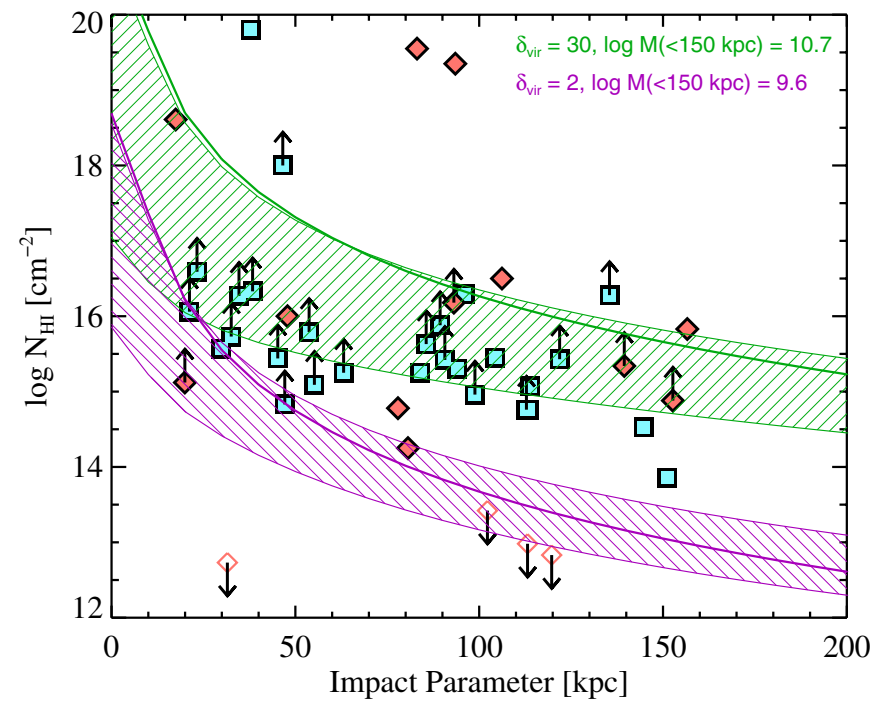

Figure 18. Figure 7 repeated with simple physical models included. The green and purple curve families show the regions covered by models with $\delta_{0}=30$ and 2 , respectively. All models have $R_{\mathrm{vir}}=350 \mathrm{kpc}$ and $\alpha=-2$. In each shaded region, models with constant temperature $T=10^{4} \mathrm{~K}$ define the upper bound and $T=10^{5} \mathrm{~K}$ the lower bound. The intermediate solid heavy curves in each set assume the same density profile but include the density-dependent equilibrium temperature shown in Figure 17 (for solar metallicity).

(A color version of this figure is available in the online journal.)

( $b \simeq 15-40 \mathrm{~km} \mathrm{~s}^{-1}$ ) could exist at or near the equilibrium curves for much longer times, remaining stable long enough to be the most commonly detected component of the CGM. A hot medium with $T \sim 10^{6} \mathrm{~K}$ could persist for long times if the density is low ( $\delta \sim 10-100)$, but would then have neutral fractions and $\mathrm{H}$ i columns and linewidths that could easily evade detection; the absence of such detections is not evidence against the existence of hot halos surrounding galaxies. These models show that a cool, photoionized medium a few hundred $\mathrm{kpc}$ in extent at moderate overdensity, with a moderate degree of clumping, can plausibly recover the observed column densities and line-kinematics in a physically plausible ionization scenario.

The second part of this simple analysis combines these basic elements and revisits the more prescriptive halo models illustrated above (Section 6.1 and Figure 14). Here we assume that the gas is distributed according to a smooth power law density profile, $\rho \propto R^{\alpha}$ normalized to $\delta_{0}$ at $R_{\text {vir }}$ and $\alpha=-2$. The temperature is again held fixed with $R$ but varied in families of models. The same ionization tables are used as in the sightline analysis just above. The results of these models are shown in Figure 18. Here we show two families of models, with $\delta_{0}=2$ (purple) and $\delta_{0}=30$ (green). The lower envelope of each shaded region corresponds to $\log T=5$, the upper envelope to $\log T=4$. The heavy curves in each set take the same density profile but apply the density-dependent equilibrium temperature from Figure 17 (at solar metallicity) to set the temperature as a function of local density. The higher curves are better matches to the COS-Halos data, which is roughly the expected overdensity at $R_{\text {vir }}$ for halos at this mass scale $\left(\delta_{0} \sim 30\right)$. The implied mass for these models is $M \simeq 5 \times 10^{10} M_{\odot}$ out to $150 \mathrm{kpc}$ in physical radius, comparable to the stellar masses, and apparently valid for both galaxy types. These models are too simple and our data too meager to account for clumping, non-equilibrium ionization, aspherical distributions, or any more complicated physics, but they do show that there are plausible models for a diffuse ionizing CGM around these galaxies and that such a medium has a significant total mass.

\section{SUMMARY}

COS-Halos has characterized the diffuse gas near $L \sim L^{*}$ galaxies using a new sample of QSO/galaxy pairs selected specifically for this purpose. This survey spans both starforming and passive galaxies with sightlines ranging at projected separations up to $150 \mathrm{kpc}$. This paper has presented the detailed properties of the survey design and the procedures followed in the collection and processing of the data. We have also presented results of the COS-Halos census of $\mathrm{H}$ I surrounding these $\sim L^{*}$ galaxies. The key findings of the H i survey are:

1. With detection limits at rest-frame equivalent width $W_{r} \sim$ $30-50 \mathrm{m \AA}$, or $\log N_{\mathrm{H}_{\mathrm{I}}} \sim 13$, neutral $\mathrm{H}$ is detected $100 \%$ of the time around star-forming galaxies and $75 \%$ of the time around passive galaxies (Figure 7) within impact parameters of $150 \mathrm{kpc}$ (physical). These detections are stronger than those typically found more than $\gtrsim 300 \mathrm{kpc}$ from galaxies, indicating that high-column density circumgalactic material is associated with the targeted galaxy at high statistical significance; weaker absorption is more broadly distributed and may not be associated directly with galaxies. We find generally good agreement between our sample and the prior studies that have examined sightlines within $200 \mathrm{kpc}$ of galaxies (Chen et al. 2001; Bowen et al. 2002; Penton et al. 2002; Wakker \& Savage 2009; Stocke et al. 2013).

2. As reported by Thom et al. (2012), there is modest but not conclusive evidence for a difference in the CGM properties of the star-forming and passive subsamples. COS-Halos shows four non-detections in the passive sample of 16 galaxies, but only (strong) detections in the $28 L^{*}$ starforming galaxies (Figures 7-9). However, the H I strengths are similar for the detections in the two subsamples when non-detections are excluded. The CGM gas mass implied by these measurements are similar for the two sub-populations (Section 6.3.3 and Figure 18). Thus we conclude that even passive galaxies are associated with strong $\mathrm{H}$ I absorption and CGM mass, though possibly at a lower frequency than star-forming galaxies.

3. Considering relative velocities between galaxies and their associated absorption, we find that most of the detected material is within approximately $\pm 200 \mathrm{~km} \mathrm{~s}^{-1}$ of the galaxy systemic velocity (Figure 10). This velocity range includes $74 \%$ of fitted components by number and $>99 \%$ of the total column density of fitted components. Strong $\mathrm{HI}_{\mathrm{I}}\left(\log N_{\mathrm{HI}} \gtrsim 16\right)$ occurs within this range $90 \%$ of the time. This range is generally within the expected escape velocity of the galaxies as calculated from their inferred DM halo masses. Conversely, weaker $\left(\log N_{\mathrm{HI}} \lesssim 15\right)$ components are seen at all relative velocities out to more than $\pm 500 \mathrm{~km} \mathrm{~s}^{-1}$. Thus we conclude that the detected strong $\mathrm{H}_{\mathrm{I}}$ is most likely bound gravitationally to the nearby galaxy, while weaker components seen at any velocity may be associated with extended LSSs or nearby galaxies in addition to the targeted galaxies.

4. Using line-profile fits to decompose the observed profiles into resolved components, we find that the line widths range over $b=10-40 \mathrm{~km} \mathrm{~s}^{-1}$, with a few broader lines (Figure 10). These line widths indicate that most of the detected column density (and, perhaps, inferred mass) lies at temperatures of $T \lesssim 10^{5} \mathrm{~K}$, far less than would be expected for shock-heated gas in virialized halos of $\log M_{\text {halo }} \simeq 12-13$. A substantial quantity of hot $\left(\sim 10^{6} \mathrm{~K}\right)$ 
gas could be present in these halos and remain unseen owing to the strong presence of the cooler material.

5. The observed trend of $\mathrm{H}$ I strength with impact parameter (Figure 7), the tight kinematic correlation with galaxy systemic velocity (Figure 10), and the concentration of $\mathrm{H}_{\mathrm{I}}$ near the galaxies with respect to results of blind surveys out to $\sim 1 \mathrm{Mpc}$ (Figure 12) lead us to conclude that the detected material does not arise in the nearby IGM, in other galaxies, or otherwise far away from the targeted galaxies. The simplest explanation for these findings is that the detected gas is directly associated with the targeted galaxies, and probably gravitationally bound to them.

6. Comparing our line strength and width measurements to blind Hi surveys in the literature, we find a strong indication that H I column densities-and perhaps the number of absorbing clouds-evolve as sightlines get nearer to galaxies, but there is no evidence that linewidths do so (Figures 10 and 13). We interpret this lack of evolution in the linewidths as an indication that the bulk of $\mathrm{H}$ I absorption arises in gas with temperatures $T \lesssim 10^{5} \mathrm{~K}$ regardless of location. Even in galaxy halos where higher temperatures from shock-heating in virialization and/or feedback might be expected, significant amounts of cold gas remain.

7. Because of our poor knowledge of the gaseous outskirts of galaxies, it is difficult to constrain the direct contribution of gas bound to the satellites of the targeted galaxies. However, a simple analysis based on DM substructure counts indicates that to explain the column densities and kinematic extent of the detected absorption would require gas to be commonly associated with very small subhalos $\left(\lesssim 10 \mathrm{~km} \mathrm{~s}^{-1}\right)$ that are typical of dwarf spheroidal satellites not known to retain gas at the observed column densities. We conclude that gas directly bound to satellites may contribute to the detected absorption but is not likely to be the primary source.

The picture that emerges from these findings is of a diffuse, cool CGM surrounding nearly all galaxies at $\sim L^{*}$, regardless of type. This CGM is composed mainly of gas at temperatures expected for low densities in photoionizing conditions. Its internal motions may be turbulent (adding some non-thermal broadening) but its bulk flows are insufficient to unbind it from the galaxy. This medium exists around both star-forming and passive galaxies, though the latter may possess a lower volume filling factor of cold gas that projects a lower areal covering fraction owing to generally higher halo gas temperatures or gas removal during the transition to passive evolution. The ionization correction that should be applied to these values of $N_{\mathrm{H} \text { I }}$ are the critical factor in obtaining total gas surface density measurements for the CGM; ionization factions cannot be measured with $\mathrm{H}_{\mathrm{I}}$ itself but can be inferred from associated metal lines from species over a range of ionization potential. An empirical characterization of the metal-lines in the COSHalos survey has been presented recently by Werk et al. (2013). A forthcoming paper will present a combined analysis of the HI and metals in terms of ionization models and physical interpretations. Other follow-up studies will consider the relationship between $\mathrm{HI}_{\mathrm{I}}$, the low-ionization metals, and the O vi results presented by Tumlinson et al. (2011a). These measurements are only one piece of the CGM puzzle, but as the $\mathrm{H}$ I traces the dominant component of the gas, the hydrogen, these measurements provide a critical basis for our planned studies of the ionization state, metallicity, and mass of the detected CGM.
Support for program GO11598 was provided by NASA through a grant from the Space Telescope Science Institute, which is operated by the Association of Universities for Research in Astronomy, Inc., under NASA contract NAS 5-26555. Some of the data presented herein were obtained at the W. M. Keck Observatory, which is operated as a scientific partnership among the California Institute of Technology, the University of California and the National Aeronautics and Space Administration. T.M.T. appreciates support from NSF grant AST-0908334. M.S.P. acknowledges support from the Southern California Center for Galaxy Evolution, a multi-campus research program funded by the University of California Office of Research. Thanks to Derck Massa for delivering the COS flats and to Ed Jenkins for processing them for our use. Thanks also to Shelley Meyett and Parviz Ghavamian for ensuring the success of the COS observations.

Facilities: HST (COS), Keck:I (LRIS, HIRES), Magellan:Clay

\section{REFERENCES}

Aracil, B., Tripp, T. M., Bowen, D. V., et al. 2006, MNRAS, 367, 139 Bahcall, J. N., Bergeron, J., Boksenberg, A., et al. 1993, ApJS, 87, 1 Bahcall, J. N., \& Spitzer, L. J. 1969, ApJL, 156, L63 Battisti, A. J., Meiring, J. D., Tripp, T. M., et al. 2012, ApJ, 744, 93 Bell, E. F., McIntosh, D. H., Katz, N., \& Weinberg, M. D. 2003, ApJS, 149, 289 Bird, S., Vogelsberger, M., Sijacki, D., et al. 2013, MNRAS, 429, 3341 Blanton, M. R., \& Roweis, S. 2007, AJ, 133, 734

Blanton, M. R., Schlegel, D. J., Strauss, M. A., et al. 2005, AJ, 129, 2562 Bordoloi, R., Lilly, S. J., Knobel, C., et al. 2011, ApJ, 743, 10 Bouché, N., Murphy, M. T., Kacprzak, G. G., et al. 2013, Sci, 341, 50 Bowen, D. V., Blades, J. C., \& Pettini, M. 1996, ApJ, 464, 141 Bowen, D. V., Pettini, M., \& Blades, J. C. 2002, ApJ, 580, 169 Bregman, J. N. 2007, ARA\&A, 45, 221

Cen, R., \& Ostriker, J. P. 1999, ApJ, 514, 1

Chen, H.-W., Lanzetta, K. M., Webb, J. K., \& Barcons, X. 1998, ApJ, 498, 77 Chen, H.-W., Lanzetta, K. M., Webb, J. K., \& Barcons, X. 2001, ApJ, 559, 654 Chen, H.-W., Prochaska, J. X., Weiner, B. J., et al. 2005, ApJL, 629, L25 Collins, J. A., Shull, J. M., \& Giroux, M. L. 2003, ApJ, 585, 336 Crighton, N. H. M., Hennawi, J. F., \& Prochaska, J. X. 2013, ApJL, 776, L18 Davé, R., Hernquist, L., Katz, N., \& Weinberg, D. H. 1999, ApJ, 511, 521 Dekel, A., \& Birnboim, Y. 2006, MNRAS, 368, 2 Diemand, J., Kuhlen, M., \& Madau, P. 2007, ApJ, 667, 859

Dixon, W. V. 2010, Cosmic Origins Spectrograph Instrument Handbook (Baltimore, PA: STScl)

Dove, J. B., \& Shull, J. M. 1994, ApJ, 423, 196

Feigelson, E. D., \& Nelson, P. I. 1985, ApJ, 293, 192

Fox, A. J., Savage, B. D., \& Wakker, B. P. 2006, ApJS, 165, 229

Fukugita, M., Hogan, C. J., \& Peebles, P. J. E. 1998, ApJ, 503, 518

Gauthier, J.-R., Chen, H.-W., \& Tinker, J. L. 2010, ApJ, 716, 1263

Gehrels, N. 1986, ApJ, 303, 336

Ghavamian, P., Aloisi, A., Lennon, D., et al. 2009, Preliminary Characterization of the Post-Launch Line Spread Function of COS, Technical Report

Green, J. C., Froning, C. S., Osterman, S., et al. 2012, ApJ, 744, 60

Haardt, F., \& Madau, P. 2001, in Clusters of Galaxies and the High Redshift

Universe Observed in X-Rays, 36th Rencontres de Moriond, ed. D. M. Neumann \& J. T. T. Van

Herenz, P., Richter, P., Charlton, J. C., \& Masiero, J. R. 2013, A\&A, 550, 87 Howk, J. C., \& Consiglio, S. M. 2012, ApJ, 759, 97

Jannuzi, B. T., Bahcall, J. N., Bergeron, J., et al. 1998, ApJS, 118, 1

Kalberla, P. M. W., \& Dedes, L. 2008, A\&A, 487, 951

Kalberla, P. M. W., \& Kerp, J. 2009, ARA\&A, 47, 27

Kereš, D., Katz, N., Weinberg, D. H., \& Davé, R. 2005, MNRAS, 363, 2 Kollmeier, J. A., Weinberg, D. H., Davé, R., \& Katz, N. 2003, ApJ, 594, 75 Lanzetta, K. M., Bowen, D. V., Tytler, D., \& Webb, J. K. 1995, ApJ, 442, 538 Lehner, N., Howk, J. C., Thom, C., et al. 2012, MNRAS, 424, 2896

Lehner, N., Howk, J. C., Tripp, T. M., et al. 2013, ApJ, 770, 138

Maller, A. H., \& Bullock, J. S. 2004, MNRAS, 355, 694

Maloney, P. 1993, ApJ, 414, 41

Martin, D. C., Fanson, J., Schiminovich, D., et al. 2005, ApJL, 619, L1 McIntosh, D. H., Guo, Y., Hertzberg, J., et al. 2008, MNRAS, 388, 1537 Meiring, J. D., Tripp, T. M., Prochaska, J. X., et al. 2011, ApJ, 732, 35 Mo, H. J., \& Miralda-Escude, J. 1996, ApJ, 469, 589 
Morris, S. L., Weymann, R. J., Dressler, A., et al. 1993, ApJ, 419, 524 Morris, S. L., Weymann, R. J., Savage, B. D., \& Gilliland, R. L. 1991, ApJL, 377, L21

Moster, B. P., Somerville, R. S., Maulbetsch, C., et al. 2010, ApJ, 710, 903

Murphy, E. M., Lockman, F. J., \& Savage, B. D. 1995, ApJ, 447, 642

Oliveira, C., Beland, S., \& Keyes, T. 2010, COS ISR 2010-06(v1), 1

Oyaizu, H., Lima, M., Cunha, C. E., et al. 2008, ApJ, 674, 768

Penton, S. V., Shull, J. M., \& Stocke, J. T. 2000a, ApJ, 544, 150

Penton, S. V., Stocke, J. T., \& Shull, J. M. 2000b, ApJS, 130, 121

Penton, S. V., Stocke, J. T., \& Shull, J. M. 2002, ApJ, 565, 720

Penton, S. V., Stocke, J. T., \& Shull, J. M. 2004, ApJS, 152, 29

Persic, M., \& Salucci, P. 1992, MNRAS, 258, 14P

Prochaska, J. X., Hennawi, J. F., \& Simcoe, R. A. 2013, ApJL, 762, L19

Prochaska, J. X., Weiner, B., Chen, H.-W., et al. 2011a, ApJS, 193, 28

Prochaska, J. X., Weiner, B., Chen, H.-W., et al. 2011b, ApJ, 740, 91

Prochter, G. E., Prochaska, J. X., \& Burles, S. M. 2006, ApJ, 639, 766

Richter, P., Savage, B. D., Sembach, K. R., \& Tripp, T. M. 2006, A\&A, 445,827

Rudie, G. C., Steidel, C. C., Trainor, R. F., et al. 2012, ApJ, 750, 67

Savage, B. D., Lehner, N., \& Narayanan, A. 2011a, ApJ, 743, 180

Savage, B. D., Narayanan, A., Lehner, N., \& Wakker, B. P. 2011b, ApJ, 731, 14

Savage, B. D., \& Sembach, K. R. 1991, ApJ, 379, 245
Schneider, D. P., Hall, P. B., Richards, G. T., et al. 2007, AJ, 134, 102

Sembach, K. R., Wakker, B. P., Savage, B. D., et al. 2003, ApJS, 146, 165

Shull, J. M., Tumlinson, J., \& Giroux, M. L. 2003, ApJL, 594, L107

Sijacki, D., Vogelsberger, M., Kereš, D., et al. 2012, MNRAS, 424, 2999

Steidel, C. C., Erb, D. K., Shapley, A. E., et al. 2010, ApJ, 717, 289

Stocke, J. T., Keeney, B. A., Danforth, C. W., et al. 2013, ApJ, 763, 148

Thom, C., \& Chen, H.-W. 2008, ApJS, 179, 37

Thom, C., Tumlinson, J., Werk, J. K., et al. 2012, ApJL, 758, L41

Thom, C., Werk, J. K., Tumlinson, J., et al. 2011, ApJ, 736, 1

Tilton, E. M., Danforth, C. W., Shull, J. M., \& Ross, T. L. 2012, ApJ, 759, 112

Tripp, T. M., Giroux, M. L., Stocke, J. T., et al. 2001, ApJ, 563, 724

Tripp, T. M., Lu, L., \& Savage, B. D. 1998, ApJ, 508, 200

Tripp, T. M., Sembach, K. R., Bowen, D. V., et al. 2008, ApJS, 177, 39

Tumlinson, J., Thom, C., Werk, J. K., et al. 2011a, Sci, 334, 948

Tumlinson, J., Werk, J. K., Thom, C., et al. 2011b, ApJ, 733, 111

Wakker, B. P. 1991, A\&A, 250, 499

Wakker, B. P., \& Savage, B. D. 2009, ApJS, 182, 378

Werk, J. K., Prochaska, J. X., Thom, C., et al. 2012, ApJS, 198, 3

Werk, J. K., Prochaska, J. X., Thom, C., et al. 2013, ApJS, 204, 17

White, S. D. M., \& Rees, M. J. 1978, MNRAS, 183, 341 